Report number: UT-Komaba 96-16

\title{
T-Duality Transformation and Universal Structure of Non-Critical String Field Theory
}

\author{
Takashi Asatani * Tsunehide Kuroki ${ }^{\dagger} \quad$ Yuji Okawa ${ }^{\ddagger} \S$ \\ Fumihiko Sugino ${ }^{* *}$ Tamiaki Yoneya ${ }^{\dagger \dagger}$ \\ Institute of Physics, University of Tokyo, Komaba, Meguro-ku, Tokyo 153, Japan
}

\begin{abstract}
We discuss a T-duality transformation for the $c=1 / 2$ matrix model for the purpose of studying duality transformations in a possible toy example of nonperturbative frameworks of string theory. Our approach is to first investigate the scaling limit of the Schwinger-Dyson equations and the stochastic Hamiltonian in terms of the dual variables and then compare the results with those using the original spin variables. It is shown that the $c=1 / 2$ model in the scaling limit is T-duality symmetric in the sphere approximation. The duality symmetry is however violated when the higher-genus effects are taken into account, owing to the existence of global $\boldsymbol{Z}_{2}$ vector fields corresponding to nontrivial homology cycles. Some universal properties of the stochastic Hamiltonians which play an important role in discussing the scaling limit and have been discussed in a previous work by the last two authors are refined in both the original and dual formulations. We also report a number of new explicit results for various amplitudes containing macroscopic loop operators.
\end{abstract}

11.25.pm, 11.25.Sq

Typeset using REVTEX

*asatani@hep1.c.u-tokyo.ac.jp

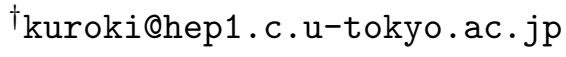

łokawa@hep1.c.u-tokyo.ac.jp

$\S$ JSPS Research Fellow

**Present address: KEK Theory Group, Tsukuba, Ibaraki 305, Japan and sugino@theory ·kek. jp

$\dagger^{\dagger}$ tam@hep1.c.u-tokyo.ac.jp 


\section{INTRODUCTION}

Recently, dual transformations in various different forms are playing increasingly important roles in string theory. Historically, one of the first useful examples of the dual transformations was the Kramers-Wannier duality [1] for the two-dimensional Ising model on the square lattice, which allows us to locate exactly the critical temperature for the order-disorder phase transition. The familiar T-dual transformation in closed string theories compactified on a torus is basically the same as the Kramers-Wannier dual transformation on discretized world sheets for a compact $U(1)$-spin model (an XY model with a Villaintype action, see, e.g. [2] and Appendix A), where the circle as the target space becomes the spin variable and the role of the compactification radius is replaced by the inverse temperature. A remarkable feature of the string T-duality is that the duality symmetry is exact for any world sheet of arbitrary fixed genus, owing to the symmetrical roles [3] played by the momentum and winding modes of closed strings. On the other hand, the conjectured S-duality transformation of string theory is a duality with respect to the target space itself and is a generalization of the electric-magnetic duality of gauge field theories, exchanging the coupling constant and its inverse as required by the Dirac condition. Complete understanding of all the duality transformations, especially the S-dualities, in string theory would obviously require some nonperturbative framework. The recent developments [4] of the idea of Dirichlet branes in fact suggest that the T-dual transformations should also play some important roles in nonperturbative physics.

In view of this situation, it seems worthwhile to formulate the duality symmetries in a simplest setting for nonperturbative string theory. Only known effective formulation of string theory which is in principle nonperturbative is the matrix model corresponding to $c \leq 1$ conformal matter coupled with the world-sheet metric. In the present paper, as a simple exercise towards nonperturbative understanding of dualities in string theory, we study the T-dual transformation of the double-scaled two-matrix model which is the Ising model at the critical point on random surfaces. Naively, we expect that the model should be self-dual, since the Ising system sits precisely at the critical point. We will in fact argue that the self-duality is valid in the sphere approximation. In higher genera, however, the duality symmetry is violated owing to the effect of nontrivial homology cycles. Here, there is no analogue of the symmetry between the momentum and winding modes which is responsible for the exact duality symmetry in the torus-compactified model. Rather, the dual theory must be described by vector fields residing on links. Only in the special case of sphere, the vector fields can be expressed in terms of the dual spin fields.

Another motivation for studying this model is to further pursue the remarkable universal structure of non-critical string field theory [5] which is relevant in taking the scaling limit and for studying background independence, using both the original and dual variables and to complement the results of the previous work [6] by two of the present authors.

The plan of the present paper is as follows. In the next section, we first explain the Kramers-Wannier duality transformation of the two-matrix model. Although the dual transformation of the two-matrix model has been discussed in the literature. we here explain

\footnotetext{
${ }^{1}$ In particular, we mention the reference [7] in which the disk amplitude in the dual basis was
} 
some elementary details of the construction for the purpose of making our interpretation to be sufficiently definite. In particular, we emphasize that the nontrivial homology cycles generally violate the duality symmetry between the spin and dual-spin variables, since they allow global vector fields. In section III, following the method of the previous paper [6], we derive the stochastic Hamiltonian [8] in terms of the dual variables. In section IV, we proceed to discuss the double scaling limit of the dual matrix model by examining the structure of the Schwinger-Dyson equations and the stochastic Hamiltonian using the dualmatrix variables. We will explicitly evaluate several lower-point correlators and determine their scaling behaviors. In section $\mathrm{V}$, based on the results of the previous section, we exhibit commutativity property between the operator mixing and the merging-splitting interaction of the macroscopic loop fields, in terms of both the original and dual matrix variables. The results generalize those of the previous paper [6]. In section VI, we discuss the duality symmetry of the stochastic Hamiltonians. We show that the Hamiltonians are duality symmetric in the sphere approximation, although they are not symmetric for higher genus owing to the existence of global vector fields on the world sheets with nontrivial homology cycles, in conformity with the discussion in section II. Section VII is devoted to concluding remarks. In Appendix A, we present a very brief discussion on how the exact T-duality symmetry is understood as the Kramers-Wannier duality on arbitrary discretized world sheets. Some of the computational details are discussed in Appendices B and C.

\section{KRAMERS-WANNIER DUAL TRANSFORMATION IN THE TWO-MATRIX MODEL}

The partition function we study in the present paper is the $N \times N$ hermitian two-matrix model given by

$$
\begin{gathered}
Z=\int d^{N^{2}} A d^{N^{2}} B \exp [-S(A, B)] \\
S(A, B)=N \operatorname{tr}\left(\frac{1}{2} A^{2}+\frac{1}{2} B^{2}-c A B-\frac{g}{3} A^{3}-\frac{g}{3} B^{3}\right) .
\end{gathered}
$$

As is well known, this represents an Ising system on random surface, where the Boltzmann factors are given by $\frac{1}{1-c^{2}}$ and $\frac{c}{1-c^{2}}$ for the nearest neighbor Ising links, connecting the spin sites with the same or opposite spins, respectively.

The inverse temperature $\beta$ for the Ising system is then

$$
\beta=-\frac{1}{2} \ln c
$$

Note that the vertices of Feynman diagrams are nothing but the surface elements in the standard random surface interpretation and the Ising spins live on the center of the surface elements. Hence the Ising links just correspond to the propagators of Feynman diagrams.

derived in the sphere approximation. 
The geometrical Boltzmann factor for the elementary surface element is $g \sim \mathrm{e}^{-\lambda}$ with $\lambda$ being the bare cosmological constant. In what follows, we use the terminologies, 'sites', 'links', in the sense of lattice spin systems, and 'vertices', 'edges', in the sense of triangulations of the random surface.

Now let us consider the Kramers-Wannier dual transformation for this system. A standard way of performing the dual transformation is to first reinterpret the Boltzmann factors $\mathrm{e}^{ \pm \beta}$ for the links as those for the dual links $\mathrm{e}^{ \pm \tilde{\beta}}$ by making the $\boldsymbol{Z}_{2}$ Fourier transformation as

$$
\begin{aligned}
\mathrm{e}^{\beta} & =K\left(\mathrm{e}^{\tilde{\beta}}+\mathrm{e}^{-\tilde{\beta}}\right) \\
\mathrm{e}^{-\beta} & =K\left(\mathrm{e}^{\tilde{\beta}}-\mathrm{e}^{-\tilde{\beta}}\right)
\end{aligned}
$$

where $K$ is an overall normalization constant $K=1 /\left(\mathrm{e}^{2 \tilde{\beta}}-\mathrm{e}^{-2 \tilde{\beta}}\right)^{1 / 2}$. This fixes the dual inverse temperature $\tilde{\beta}$ as

$$
\tilde{\beta}=-\frac{1}{2} \ln \tilde{c}
$$

with

$$
\tilde{c}=\frac{1-c}{1+c}
$$

For a fixed lattice, the Boltzmann factor $\exp \tilde{\beta} v_{\tilde{\ell}}$ would correspond to dual links $\tilde{\ell}$ with parallel ("stick": $v_{\tilde{\ell}}=+1$ ) dual spins or anti-parallel ("flip": $v_{\tilde{\ell}}=-1$ ) dual spins, respectively. In the present case, however, we will not introduce dual spin variables for reasons explained below. Usually, the dual spin variables are introduced as the solutions for the constraint for the dual link variables after integrating over the original spin configurations. The constraints take the form

$$
\prod_{\tilde{\ell} \subset \tilde{C}} v_{\tilde{\ell}}=1
$$

for all elementary (namely smallest) closed loops $\tilde{C}$ of dual links encircling the spin sites of the original lattice. The solution for these constraints is locally given as $v_{\tilde{\ell}}=\tilde{s}_{\tilde{\ell}_{1}} \cdot \tilde{s}_{\tilde{\ell}_{2}}$ by introducing the dual spin variables $\tilde{s}_{\tilde{\ell}_{1}}, \tilde{s}_{\tilde{\ell}_{2}}(= \pm 1)$ at the center of all dual surface elements. The fixed square lattice with Ising spins is then duality symmetric under the interchange $\tilde{\beta} \leftrightarrow \beta$.

For the present model, there are at least two reasons that the system cannot in general be duality symmetric. The first reason is due to the nontrivial homology cycles of the general random surfaces. The constraint (2.8) allows nontrivial solutions which cannot be reduced to the product of the dual spin variables, owing to the existence of global $\boldsymbol{Z}_{2}$ vector fields, associated with the nontrivial homology cycles. For this reason, we will treat the dual transformed model by dealing only with the dual link variables without introducing the dual spin variables explicitly. Secondly, the random surfaces of the two-matrix model (2.2) are discretized by triangles corresponding to the cubic vertices. Hence the original spin sites have always three links attached. After the above dual transformation, the dual spin sites can now have an arbitrary number of dual links attached. For this reason, the critical point cannot be fixed to be the self-dual point in contrary to the case of the fixed square lattice. 
We can however expect that in the continuum limit this difference might be washed out and the model should exhibit partial duality symmetry in some situation where we can neglect the effect of nontrivial homology cycles. We will argue in later sections that this is indeed the case.

Now in terms of the matrix fields $A, B$, the Boltzmann factors for the original Ising links are related to the bare propagators as

$$
\begin{aligned}
\langle A A\rangle_{0}= & \langle B B\rangle_{0}=L \mathrm{e}^{\beta}, \\
& \langle A B\rangle_{0}=L \mathrm{e}^{-\beta}
\end{aligned}
$$

with $L=\sqrt{c} /\left(1-c^{2}\right)$, where $\langle\cdot\rangle_{0}$ indicates the expectation value with the quadratic action. Substituting the Fourier transformation (2.4) and (2.5), we have

$$
\begin{aligned}
\langle A A\rangle_{0}=\langle B B\rangle_{0} & =\frac{1}{2 \sqrt{1-c^{2}}}\left(\mathrm{e}^{\tilde{\beta}}+\mathrm{e}^{-\tilde{\beta}}\right), \\
\langle A B\rangle_{0} & =\frac{1}{2 \sqrt{1-c^{2}}}\left(\mathrm{e}^{\tilde{\beta}}-\mathrm{e}^{-\tilde{\beta}}\right) .
\end{aligned}
$$

This clearly shows that the dual transformation is performed simply by changing to the new matrix fields $X$ and $Y$, which diagonalize the kinetic term of the original action, as

$$
\begin{aligned}
& X=\frac{1}{\sqrt{2}}(A+B) \\
& Y=\frac{1}{\sqrt{2}}(A-B)
\end{aligned}
$$

whose propagators just give the Boltzmann factors for the dual model,

$$
\begin{gathered}
\langle X X\rangle_{0}=\frac{1}{\sqrt{1-c^{2}}} \mathrm{e}^{\tilde{\beta}}=\frac{1}{1-c}, \\
\langle Y Y\rangle_{0}=\frac{1}{\sqrt{1-c^{2}}} \mathrm{e}^{-\tilde{\beta}}=\frac{1}{1+c} .
\end{gathered}
$$

The new matrix fields $X, Y$ can be regarded as living on the dual vertices (surface elements or triangles of the original lattice) but their propagators are associated with the dual Ising links (dual to the edges of the dual surface elements), having one-to-one correspondence with original Ising links. Thus they should not be confused with the dual spin variables themselves which are supposed to live on dual-spin sites, namely, the center of the dual surface elements corresponding to the vertices of the original random surfaces.

In terms of the new matrix fields, the partition function is $(\hat{g}=g / \sqrt{2})$

$$
\begin{gathered}
Z=\int d^{N^{2}} X d^{N^{2}} Y \exp \left[-S_{D}(X, Y)\right] \\
S_{D}(X, Y)=N \operatorname{tr}\left(\frac{1-c}{2} X^{2}+\frac{1+c}{2} Y^{2}-\frac{\hat{g}}{3}\left(X^{3}+3 X Y^{2}\right)\right) .
\end{gathered}
$$

In comparison with the original representation, this representation itself does not show any trace of possible dual symmetry, reflecting the situation explained above. This simply defines 
a mapping of an Ising model on random surfaces to a special case of the $\mathrm{O}(\mathrm{n})$ model as has been discussed by several authors [9].

The homological property we have discussed can now be described by the configurations of closed loops formed by the $Y$ field. The global $\boldsymbol{Z}_{2}$ vector fields correspond to the closed $Y$-loops winding around the nontrivial homological cycles of the surfaces. The $\boldsymbol{Z}_{2}$ nature comes from the fact that even number of closed $Y$-loops winding around nontrivial homology cycles are continuously deformed into null closed $Y$-loop. In the case of sphere, there is no nontrivial homology cycles and hence we can introduce dual $\boldsymbol{Z}_{2}$ spin variables which reside on the domains bounded by closed $Y$-loops. The existence of such closed $Y$-loops winding around nontrivial cycles forbids to define dual spin domains globally. Equivalently, the Hamiltonian description of the time development of the random surfaces necessarily requires us to introduce the observables containing odd number of the $Y$-fields.

Let us next discuss the basis of string field representations used in the present paper. In the previous work, we used the component expansion of string fields with respect to the number of spin domains. In the dual formulation, the basis which can be compared with this is the expansion with respect to the number of the $Y$-fields. Thus the observables we consider are of the following type:

$$
\begin{gathered}
\frac{1}{N} \operatorname{tr}\left(\frac{1}{\xi-X}\right) \equiv \Psi_{X}(\xi) \\
\frac{1}{N} \operatorname{tr}\left(\frac{1}{\xi_{1}-X} Y\right) \equiv \Psi_{1}\left(\xi_{1}\right), \\
\frac{1}{N} \operatorname{tr}\left(\frac{1}{\xi_{1}-X} Y \frac{1}{\xi_{2}-X} Y\right) \equiv \Psi_{2}\left(\xi_{1}, \xi_{2}\right), \\
\frac{1}{N} \operatorname{tr}\left(\frac{1}{\xi_{1}-X} Y \frac{1}{\xi_{2}-X} Y \frac{1}{\xi_{3}-X} Y\right) \equiv \Psi_{3}\left(\xi_{1}, \xi_{2}, \xi_{3}\right),
\end{gathered}
$$

etc.

We note that if we could have neglected the closed $Y$-loops winding around the nontrivial homology cycles, $\Psi$ 's with odd number of $Y$ 's were not necessary. We would then have the interpretation of these quantities in terms of domains with respect to dual spins, and $\Psi_{2 n}$ would correspond to the component of the field with $n$ pairs of domains. Note that the matrix $Y$ just represents the domain boundaries of the dual-spin interpretation. Thus, in studying the duality symmetry, $\Psi_{2 n}$ should be substituted in place of the observables considered in the previous paper, namely,

$$
\Phi_{n}\left(\zeta_{1}, \sigma_{1}, \cdots, \zeta_{n}, \sigma_{n}\right) \equiv \frac{1}{N} \operatorname{tr}\left(\prod_{i}^{n} \frac{1}{\zeta_{i}-A} \frac{1}{\sigma_{i}-B}\right)
$$

after an appropriate symmetrization with respect to two matrices $A$ and $B$, taking into account the fact that the $\Psi_{2 n}$ does not discriminate global dual spin directions. A detailed 
discussion of the duality symmetry of the stochastic Hamiltonian will be presented later in section VI.

From the above discussion, it is clear at least conceptually that the dual transformation is nothing but a change of the basis of the string fields between $(2.23)$ and $(2.19) \sim(2.22)$ etc. I However, it turns out that performing this transformation directly for the string fields in the continuum limit is technically very difficult, because the process of taking the continuum limit involves an intricate operator mixing and does not seem to be commutative with the above change of the basis. Thus the question of the T-duality symmetry for the Ising model on the random surface is quite nontrivial. The approach in the present paper will therefore be somewhat indirect such that we perform an independent study of the scaling limit of the Schwinger-Dyson equations and the stochastic Hamiltonian using the dual basis and compare the results with those obtained using the original basis. One practical virtue of this approach would be that the ranges of the components where we can compute correlation functions explicitly are different from each other depending on different bases, and hence our results are useful for showing the universal nature of the structure of, say, the stochastic Hamiltonians, as has been emphasized in the previous work [6]. Presenting such a piece of evidence for the universal structure of the string field theories is another purpose of the present paper.

Finally, we here fix our notations in what follows. As introduced above, the basis operators are $\Psi_{n}$. We denote the expectation values of $\Psi_{n}$ in the sphere approximation by $V^{(n)}(\cdot)=\left\langle\Psi_{n}(\cdot)\right\rangle_{0}$ with the same arguments and suffices. We have used the notation $W^{(2 n)}$ for $\left\langle\Phi_{n}\right\rangle$ in the case of the original spin formulation [6].

\section{STOCHASTIC HAMILTONIAN IN THE DUAL FORMALISM}

Let us first derive the stochastic Hamiltonian using the dual basis of the observables $\Psi$ 's introduced in the previous section. The generating functional of the dual two-matrix model is defined by

$$
\begin{gathered}
Z[K]=\frac{1}{Z} \int d^{N^{2}} X d^{N^{2}} Y \mathrm{e}^{-S_{D}} \mathrm{e}^{K \cdot \Psi}, \\
Z=\int d^{N^{2}} X d^{N^{2}} Y \mathrm{e}^{-S_{D}}, \\
K \cdot \Psi=\int \frac{d \xi}{2 \pi i} K_{X}(\xi) \Psi_{X}(\xi)+\sum_{n=1}^{\infty} \int \prod_{i=1}^{n} \frac{d \xi_{i}}{2 \pi i} K_{n}\left(\xi_{1}, \cdots, \xi_{n}\right) \Psi_{n}\left(\xi_{1}, \cdots, \xi_{n}\right),
\end{gathered}
$$

where and in what follows the integral contour, unless specified otherwise, runs parallel to the imaginary axis towards the positive imaginary infinity on the right hand side of all the pole singularities of the observables $\Psi_{X}, \Psi_{n}$.

\footnotetext{
${ }^{2}$ We here mention a paper [10] which has discussed the T-duality in the framework of covariant string field theories of critical bosonic string theory.
} 
As discussed in the previous paper [6], the Stochastic Hamiltonian is derived from the identity,

$$
0=-\int d^{N^{2}} X d^{N^{2}} Y \sum_{\alpha=1}^{N^{2}}\left[\frac{\partial}{\partial X_{\alpha}} \mathrm{e}^{-S} \frac{\partial}{\partial X_{\alpha}}+\frac{\partial}{\partial Y_{\alpha}} \mathrm{e}^{-S} \frac{\partial}{\partial Y_{\alpha}}\right] \mathrm{e}^{K \cdot \Psi} .
$$

The notations $X_{\alpha}, Y_{\alpha}$ denote the components of the hermitian matrix in the expansion in terms of the basis $\left\{t_{\alpha}\right\}\left(\operatorname{tr}\left(t_{\alpha} t_{\beta}\right)=\delta_{\alpha \beta}\right)$. The derivative operator inside the integral may be regarded as the Laplacian operator for the present model. Expressed as a functional differential equation in terms of the sources, this takes the form

$$
\begin{gathered}
0=\mathcal{H} Z[K] \\
\mathcal{H}=-K \cdot\left(\mathcal{K} \frac{\delta}{\delta K}\right)-K \cdot\left(\frac{\delta}{\delta K} \vee \frac{\delta}{\delta K}\right)-\frac{1}{N^{2}} K \cdot\left(K \cdot\left(\wedge \frac{\delta}{\delta K}\right)\right)-K \cdot T .
\end{gathered}
$$

Here the functional derivative $\frac{\delta}{\delta K_{n}}$ acting on the source terms is defined as

$$
\frac{\delta K_{m}\left(\xi_{1}^{\prime}, \cdots, \xi_{m}^{\prime}\right)}{\delta K_{n}\left(\xi_{1}, \cdots, \xi_{n}\right)}=\delta_{m, n} \frac{1}{n}(2 \pi i)^{n} \sum_{c} \delta\left(\xi_{1}-\xi_{c(1)}^{\prime}\right) \cdots \delta\left(\xi_{n}-\xi_{c(n)}^{\prime}\right)
$$

Reflecting the cyclic symmetry of $\Psi_{n}$ with respect to its variables $\left\{\xi_{i}\right\}$, the summation in the right hand side is over cyclic permutations $c(i)$ of the indices $i=1,2, \ldots, n$ of $\xi_{i}^{\prime}$. The origin and definition of each term of the Hamiltonian (3.6) are explained below.

1. The first term, "kinetic term" $K \cdot\left(\mathcal{K} \frac{\delta}{\delta K}\right)$, comes from the product of the first derivatives of the source term and the action $(n=1,2, \ldots)$,

$$
\begin{aligned}
\left(\mathcal{K} \frac{\delta}{\delta K}\right)_{X}(\xi)= & \partial_{\xi}\left[\xi(1-c-\hat{g} \xi) \frac{\delta}{\delta K_{X}(\xi)}\right]-\hat{g} \oint \frac{d \xi^{\prime}}{2 \pi i} \partial_{\xi} \frac{\delta}{\delta K_{2}\left(\xi, \xi^{\prime}\right)}, \\
\left(\mathcal{K} \frac{\delta}{\delta K}\right)_{n}\left(\xi_{1}, \cdots, \xi_{n}\right)= & \sum_{j=1}^{n}\left[-2 c+\left(1-c-\hat{g} \xi_{j}\right) \xi_{j} \partial_{\xi_{j}}\right] \frac{\delta}{\delta K_{n}\left(\xi_{1}, \cdots, \xi_{n}\right)} \\
& +\sum_{j=1}^{n} \hat{g} \oint \frac{d \xi}{2 \pi i} \frac{\delta}{\delta K_{n+2}\left(\xi_{1}, \cdots, \xi_{j-1}, \xi_{j}, \xi, \xi_{j}, \xi_{j+1}, \cdots, \xi_{n}\right)} \\
& -\sum_{j=1}^{n} \hat{g} \oint \frac{d \xi}{2 \pi i} \frac{\delta}{\delta K_{n}\left(\xi_{1}, \cdots, \xi_{j-1}, \xi, \xi_{j+1}, \cdots, \xi_{n}\right)},
\end{aligned}
$$

where the integral $\oint$ is over a closed curve encircling all the pole singularities of the observables $\Psi$ 's. The structure of the kinetic term is slightly different from that with the original variables $A, B$. If for the moment we allow ourselves to use the dual-spin language for convenience, this contains a part which measures the number of dual-spin flip links, corresponding to the $Y$-matrices, in addition to a part measuring the length of each domain. The other parts describe the infinitesimal motion of string loops, changing their lengths and/or dual spin directions. 
2. The second term , $K \cdot\left(\frac{\delta}{\delta K} \vee \frac{\delta}{\delta K}\right)$, of (3.6) comes from the second derivative of the source term $K \cdot \Psi$ and represents processes where a loop splits into two. The symbol $\left(\frac{\delta}{\delta K} \vee \frac{\delta}{\delta K}\right)_{I}$ stands for the spin configurations of the resultant two loops from a single loop with spin configuration $I$.

$$
\begin{aligned}
& \left(\frac{\delta}{\delta K} \vee \frac{\delta}{\delta K}\right)_{X}(\xi)=-\partial_{\xi} \frac{\delta^{2}}{\delta K_{X}(\xi)^{2}} \\
& \left(\frac{\delta}{\delta K} \vee \frac{\delta}{\delta K}\right)_{1}\left(\xi_{1}\right)=-2 \frac{\delta}{\delta K_{X}\left(\xi_{1}\right)} \partial_{\xi_{1}} \frac{\delta}{\delta K_{1}\left(\xi_{1}\right)}, \\
& \left(\frac{\delta}{\delta K} \vee \frac{\delta}{\delta K}\right)_{2}\left(\xi_{1}, \xi_{2}\right)=-2 \sum_{j=1}^{2} \frac{\delta}{\delta K_{X}\left(\xi_{j}\right)} \partial_{\xi_{j}} \frac{\delta}{\delta K_{2}\left(\xi_{1}, \xi_{2}\right)} \\
& +2\left(D_{z}\left(\xi_{1}, \xi_{2}\right) \frac{\delta}{\delta K_{1}(z)}\right)^{2}+2 \frac{\delta^{2}}{\delta K_{X}\left(\xi_{1}\right) \delta K_{X}\left(\xi_{2}\right)}, \\
& \left(\frac{\delta}{\delta K} \vee \frac{\delta}{\delta K}\right)_{n}\left(\xi_{1}, \cdots, \xi_{n}\right)=-2 \sum_{j=1}^{n} \frac{\delta}{\delta K_{X}\left(\xi_{j}\right)} \partial_{\xi_{j}} \frac{\delta}{\delta K_{n}\left(\xi_{1}, \cdots, \xi_{n}\right)} \\
& +2 \sum_{k<l} D_{z}\left(\xi_{k}, \xi_{l}\right) \frac{\delta}{\delta K_{l-k}\left(z, \xi_{k+1}, \cdots, \xi_{l-1}\right)} D_{\zeta}\left(\xi_{k}, \xi_{l}\right) \frac{\delta}{\delta K_{n-l+k}\left(\xi_{1}, \cdots, \xi_{k-1}, \zeta, \xi_{l+1}, \cdots, \xi_{n}\right)} \\
& -2 \sum_{j=1}^{n} \frac{\delta}{\delta K_{X}\left(\xi_{j}\right)} D_{z}\left(\xi_{j-1}, \xi_{j+1}\right) \frac{\delta}{\delta K_{n-2}\left(\xi_{1}, \cdots, \xi_{j-2}, z, \xi_{j+2}, \cdots, \xi_{n}\right)} \\
& +2 \sum_{1<l-k<n-1} D_{z}\left(\xi_{k+1}, \xi_{l}\right) \frac{\delta}{\delta K_{l-k-1}\left(z, \xi_{k+2}, \cdots, \xi_{l-1}\right)} \\
& \times D_{\zeta}\left(\xi_{k}, \xi_{l+1}\right) \frac{\delta}{\delta K_{n-l+k-1}\left(\xi_{1}, \cdots, \xi_{k-1}, \zeta, \xi_{l+2}, \cdots, \xi_{n}\right)}, \quad(n \geq 3) .
\end{aligned}
$$

Here we used the definition of the combinatorial derivative,

$$
D_{z}\left(\xi_{1}, \xi_{2}\right) f(z)=\frac{f\left(\xi_{1}\right)-f\left(\xi_{2}\right)}{\xi_{1}-\xi_{2}} .
$$

Note also that the last term of (3.10) is absent for $n=3$.

3. The third term $\frac{1}{N^{2}} K \cdot\left(K \cdot\left(\wedge \frac{\delta}{\delta K}\right)\right)$ comes from the square of the first derivative of the source term, and represents processes in which two loops merge into a single loop. The symbol $\left(\wedge \frac{\delta}{\delta K}\right)_{I, J}$ expresses the spin configuration of the resultant single loop into which two loops with spin configurations $I, J$ merge. The variables on the left hand side of a semicolon represent the variables conjugate to the lengths of domains of a loop with configuration $I$, while those on the right hand side represent the variables with configuration $J$ : 


$$
\begin{aligned}
& \left(\wedge \frac{\delta}{\delta K}\right)_{X, X}\left(\xi ; \xi^{\prime}\right)=-\partial_{\xi} \partial_{\xi^{\prime}} D_{z}\left(\xi, \xi^{\prime}\right) \frac{\delta}{\delta K_{X}(z)} \\
& \left(\wedge \frac{\delta}{\delta K}\right)_{n, X}\left(\xi_{1}, \cdots, \xi_{n} ; \xi^{\prime}\right)=\left(\wedge \frac{\delta}{\delta K}\right)_{X, n}\left(\xi^{\prime} ; \xi_{1}, \cdots, \xi_{n}\right) \quad(n=1,2,3, \cdots) \\
& =-\sum_{j=1}^{n} \partial_{\xi^{\prime}} \partial_{\xi_{j}} D_{z}\left(\xi^{\prime}, \xi_{j}\right) \frac{\delta}{\delta K_{n}\left(\xi_{1}, \cdots, \xi_{j-1}, z, \xi_{j+1}, \cdots, \xi_{n}\right)}, \\
& \left(\wedge \frac{\delta}{\delta K}\right)_{1,1}\left(\xi_{1} ; \xi_{1}^{\prime}\right)=D_{\zeta}\left(\xi_{1}, \xi_{1}^{\prime}\right) D_{z}\left(\xi_{1}, \xi_{1}^{\prime}\right) \frac{\delta}{\delta K_{2}(z, \zeta)}-D_{z}\left(\xi_{1}, \xi_{1}^{\prime}\right) \frac{\delta}{\delta K_{X}(z)}, \\
& \left(\wedge \frac{\delta}{\delta K}\right)_{n, 1}\left(\xi_{1}, \cdots, \xi_{n} ; \xi_{1}^{\prime}\right)=\left(\wedge \frac{\delta}{\delta K}\right)_{1, n}\left(\xi_{1}^{\prime} ; \xi_{1}, \cdots, \xi_{n}\right) \quad(n=2,3,4, \cdots) \\
& =\sum_{j=1}^{n} D_{z}\left(\xi_{1}^{\prime}, \xi_{j}\right) D_{\zeta}\left(\xi_{1}^{\prime}, \xi_{j}\right) \frac{\delta}{\delta K_{n+1}\left(\xi_{1}, \cdots, \xi_{j-1}, z, \zeta, \xi_{j+1}, \cdots, \xi_{n}\right)} \\
& +\sum_{j=1}^{n} D_{\zeta}\left(\xi_{j}, \xi_{1}^{\prime}\right) D_{z}\left(\xi_{j+1}, \zeta\right) \frac{\delta}{\delta K_{n-1}\left(\xi_{1}, \cdots, \xi_{j-1}, z, \xi_{j+2}, \cdots, \xi_{n}\right)}, \\
& \left(\wedge \frac{\delta}{\delta K}\right)_{n, m}\left(\xi_{1}, \cdots, \xi_{n} ; \xi_{1}^{\prime}, \cdots, \xi_{m}^{\prime}\right) \quad(n, m=2,3,4, \cdots) \\
& =\sum_{j=1}^{n} \sum_{k=1}^{m} D_{z}\left(\xi_{j}, \xi_{k}^{\prime}\right) D_{\zeta}\left(\xi_{j}, \xi_{k}^{\prime}\right) \\
& \times \frac{\delta}{\delta K_{n+m}\left(\xi_{1}, \cdots, \xi_{j-1}, z, \xi_{k+1}^{\prime}, \cdots, \xi_{m}^{\prime}, \xi^{\prime}{ }_{1}, \cdots, \xi_{k-1}^{\prime}, \zeta, \xi_{j+1}, \cdots, \xi_{n}\right)} \\
& +\sum_{j=1}^{n} \sum_{k=1}^{m} D_{z}\left(\xi_{j}, \xi_{k+1}^{\prime}\right) D_{\zeta}\left(\xi_{k}^{\prime}, \xi_{j+1}\right) \\
& \times \frac{\delta}{\delta K_{n+m-2}\left(\xi_{1}, \cdots, \xi_{j-1}, z, \xi_{k+2}^{\prime}, \cdots, \xi_{m}^{\prime}, \xi^{\prime}{ }_{1}, \cdots, \xi_{k-1}^{\prime}, \zeta, \xi_{j+2}, \cdots, \xi_{n}\right)} .
\end{aligned}
$$

4. The last term, a tadpole term, arises again from the product of the first derivative of the source term $K_{X} \cdot \Psi_{X}$ and the action, and describes the process of the annihilation of a shortest loop into nothing :

$$
K \cdot T=\int \frac{d \xi}{2 \pi i} K_{X}(\xi) \hat{g}
$$

Similarly to the Hamiltonian of the original formalism, the present dual Hamiltonian satisfies a locality property. For instance, only the loops $\Psi_{X}$ with no domain boundary can be annihilated into nothing. Also, only a single pair of domains or a single pair of the boundaries of the domains can participate in the splitting or merging processes, and other domains remain unchanged. One of the differences between the Hamiltonians in the original and dual formulations arises from the derivatives with respect to $Y$. The $Y_{\alpha}$-derivatives produce 
processes which have no counter part in the Hamiltonian in terms of the original variables. For instance, the last terms of the kinetic (3.9) and merging terms (for $I, J \geq 1$ ) in the above expressions contain the contributions from the $Y_{\alpha}$-derivatives. For the splitting interaction, the last (for $I=2,3$ ) or the last two terms (for $I \geq 4$ ) are the contributions from the $Y_{\alpha^{-}}$ derivatives. From the view point of continuum theory, these are very singular; they would be measure-zero contributions. In later sections, we will show that the terms arising from the $Y_{\alpha}$-derivatives in fact vanish in the continuum limit.

\section{SCHWINGER-DYSON EQUATIONS AND THE SCALING LIMIT OF THE STOCHASTIC HAMILTONIAN}

Now we consider the scaling limit of the Hamiltonian (3.6). To do this, we closely follow [6]. Namely, we will first identify and subtract the non-universal parts of the disk amplitudes and establish the operator mixing, and then will rewrite the Hamiltonian (3.6) in terms of the universal parts of the operators.

As we discuss in Appendix B, the original operators $\Psi_{I}$ and their universal parts $\hat{\Psi}_{I}$ are related by a linear transformation of the following form:

$$
\Psi_{I}=\sum_{J} \mathcal{M}_{I J} \hat{\Psi}_{J}+\psi_{I}, \quad I=X, 1,2, \cdots,
$$

where $\mathcal{M}_{I J}$ is a mixing matrix of upper-triangular form, $\mathcal{M}_{I J}=0$ for $I<J$, and $\psi_{I}$ is the non-universal c-number function. The upper-triangular form of $\mathcal{M}$ comes from a property of the operator mixing that the operators corresponding to simpler configurations can mix as non-universal parts in taking the continuum limit, as has been discussed in the previous work [6]. Note that $\psi_{I}$ vanishes by itself for an arbitrary odd $I$, because there exists the $\boldsymbol{Z}_{2}$ symmetry under $Y \rightarrow-Y$. The first few components of (4.1) are

$$
\begin{aligned}
\Psi_{X}(\xi)= & \hat{\Psi}_{X}(\xi)+\psi_{X}(\xi) \\
\Psi_{1}\left(\xi_{1}\right)= & \hat{\Psi}_{1}\left(\xi_{1}\right) \\
\Psi_{2}\left(\xi_{1}, \xi_{2}\right)= & \frac{2}{\sqrt{5 c}}\left(\hat{\Psi}_{X}\left(\xi_{1}\right)+\hat{\Psi}_{X}\left(\xi_{2}\right)\right)+\hat{\Psi}_{2}\left(\xi_{1}, \xi_{2}\right)+\psi_{2}\left(\xi_{1}, \xi_{2}\right), \\
\Psi_{3}\left(\xi_{1}, \xi_{2}, \xi_{3}\right)= & \frac{2}{\sqrt{5 c}}\left[-D_{\xi}\left(\xi_{1}, \xi_{2}\right) \hat{\Psi}_{1}(\xi)-D_{\xi}\left(\xi_{2}, \xi_{3}\right) \hat{\Psi}_{1}(\xi)-D_{\xi}\left(\xi_{3}, \xi_{1}\right) \hat{\Psi}_{1}(\xi)\right] \\
& +\hat{\Psi}_{3}\left(\xi_{1}, \xi_{2}, \xi_{3}\right), \\
\Psi_{4}\left(\xi_{1}, \xi_{2}, \xi_{3}, \xi_{4}\right)= & -\frac{4}{5 c}\left(D_{\xi}\left(\xi_{1}, \xi_{3}\right) \hat{\Psi}_{X}(\xi)+D_{\xi}\left(\xi_{2}, \xi_{4}\right) \hat{\Psi}_{X}(\xi)\right) \\
& -\frac{2}{\sqrt{5 c}}\left[D_{\xi}\left(\xi_{1}, \xi_{3}\right)\left(\hat{\Psi}_{2}\left(\xi, \xi_{2}\right)+\hat{\Psi}_{2}\left(\xi, \xi_{4}\right)\right)\right. \\
& \left.+D_{\xi}\left(\xi_{2}, \xi_{4}\right)\left(\hat{\Psi}_{2}\left(\xi_{1}, \xi\right)+\hat{\Psi}_{2}\left(\xi_{3}, \xi\right)\right)\right] \\
& +\hat{\Psi}_{4}\left(\xi_{1}, \xi_{2}, \xi_{3}, \xi_{4}\right)+\psi_{4}\left(\xi_{1}, \xi_{2}, \xi_{3}, \xi_{4}\right), \\
& \ldots
\end{aligned}
$$

where 


$$
\begin{aligned}
& \psi_{X}(\xi)=\frac{2 c(c+1)}{3 \hat{g}}-\frac{1}{3}\left((5 c-1) \xi+\hat{g} \xi^{2}\right), \\
& \psi_{2}\left(\xi_{1}, \xi_{2}\right)= \frac{1}{5}(1+4 \hat{s})-2 \sqrt{\frac{c}{5}}\left(\xi_{1}+\xi_{2}\right), \\
& \psi_{4}\left(\xi_{1}, \xi_{2}, \xi_{3}, \xi_{4}\right)= 1, \\
& \cdots,
\end{aligned}
$$

and $c$ is at the critical value: $c=\frac{-1+2 \sqrt{7}}{27}$, and $\hat{s}=1+\sqrt{7}$. All these results are derived explicitly in Appendix B:

Denoting the connected $k$-point correlator for the $K=0$ background as

$$
G_{I_{1}, \cdots, I_{k}}^{(k)}=\left\langle\Psi_{I_{1}} \cdots \Psi_{I_{k}}\right\rangle, \quad I_{1}, \cdots, I_{k}=X, 1,2, \cdots
$$

the generating functional takes the form

$$
Z[K]=\exp \left[K \cdot G^{(1)}+\frac{1}{2 !} K \cdot\left(K \cdot G^{(2)}\right)+\frac{1}{3 !} K \cdot\left(K \cdot\left(K \cdot G^{(3)}\right)\right)+\cdots\right] .
$$

Separation of the universal parts $\hat{G}_{I_{1}, \cdots, I_{k}}^{(k)}$ amounts to a linear transformation

$$
\begin{aligned}
G_{I}^{(1)} & =\sum_{J} \mathcal{M}_{I J} \hat{G}_{J}^{(1)}+\psi_{I}, \\
G_{I_{1}, \cdots, I_{k}}^{(k)} & =\sum_{J_{1}, \cdots, J_{k}} \mathcal{M}_{I_{1} J_{1}} \cdots \mathcal{M}_{I_{k} J_{k}} \hat{G}_{J_{1}, \cdots, J_{k}}^{(k)} \quad(k \geq 2) .
\end{aligned}
$$

Thus, on introducing the transformed source $\hat{K}$ by

$$
K_{I}=\sum_{J} \hat{K}_{J}\left(\mathcal{M}^{-1}\right)_{J I}
$$

the generating functional $\hat{Z}[\hat{K}]$ in the continuum theory is obtained by a rescaling $Z[K]=$ $\mathrm{e}^{K \cdot \psi} \hat{Z}[\hat{K}]$, giving

$$
\hat{Z}[\hat{K}]=\exp \left[\hat{K} \cdot \hat{G}^{(1)}+\frac{1}{2 !} \hat{K} \cdot\left(\hat{K} \cdot \hat{G}^{(2)}\right)+\frac{1}{3 !} \hat{K} \cdot\left(\hat{K} \cdot\left(\hat{K} \cdot \hat{G}^{(3)}\right)\right)+\cdots\right] .
$$

The Hamiltonian acting on $\hat{Z}[\hat{K}]$ now becomes

\footnotetext{
3 As we comment in (B9) in Appendix B, $\psi_{4}$ has possibly a linear term of $y$ in addition. This term affects higher components of spin flip amplitudes through the Schwinger-Dyson equations. However, since it changes only the non-universal parts of these amplitudes, it can be absorbed into the redefinition of the non-universal parts and does not change the scaling limit of the Hamiltonian.
} 


$$
\begin{aligned}
0= & \mathcal{H} \hat{Z}[\hat{K}], \\
\mathcal{H}= & -\left(\hat{K} \mathcal{M}^{-1}\right) \cdot\left(\mathcal{K}\left(\mathcal{M} \frac{\delta}{\delta \hat{K}}+\psi\right)\right)-\left(\hat{K} \mathcal{M}^{-1}\right) \cdot T \\
& -\left(\hat{K} \mathcal{M}^{-1}\right) \cdot\left(\left(\mathcal{M} \frac{\delta}{\delta \hat{K}}+\psi\right) \vee\left(\mathcal{M} \frac{\delta}{\delta \hat{K}}+\psi\right)\right) \\
& -\frac{1}{N^{2}}\left(\hat{K} \mathcal{M}^{-1}\right) \cdot\left(\left(\hat{K} \mathcal{M}^{-1}\right) \cdot\left(\wedge\left(\mathcal{M} \frac{\delta}{\delta \hat{K}}+\psi\right)\right)\right) .
\end{aligned}
$$

Next, we try to eliminate the mixing matrix from Eq. (4.9). We claim the validity of the following identities:

$$
\begin{aligned}
-\left(\hat{K} \mathcal{M}^{-1}\right) \cdot\left(\mathcal{K}\left(\mathcal{M} \frac{\delta}{\delta \hat{K}}+\psi\right)\right)-\left(\hat{K} \mathcal{M}^{-1}\right) \cdot T \\
-\left(\hat{K} \mathcal{M}^{-1}\right) \cdot\left(\left(\mathcal{M} \frac{\delta}{\delta \hat{K}}+\psi\right) \vee\left(\mathcal{M} \frac{\delta}{\delta \hat{K}}+\psi\right)\right) \\
\quad-\frac{1}{N^{2}}\left(\hat{K} \mathcal{M}^{-1}\right) \cdot\left(\left(\hat{K} \mathcal{M}^{-1}\right) \cdot(\wedge \psi)\right) \\
=-\hat{K} \cdot\left(\mathcal{F} \frac{\delta}{\delta \hat{K}}\right)-\left(\hat{K} \mathcal{M}^{-1}\right) \cdot\left(\left(\mathcal{M} \frac{\delta}{\delta \hat{K}}\right) \vee\left(\mathcal{M} \frac{\delta}{\delta \hat{K}}\right)\right), \\
\left(\hat{K} \mathcal{M}^{-1}\right) \cdot\left(\left(\mathcal{M} \frac{\delta}{\delta \hat{K}}\right) \vee\left(\mathcal{M} \frac{\delta}{\delta \hat{K}}\right)\right)=\hat{K} \cdot\left(\frac{\delta}{\delta \hat{K}} \vee \frac{\delta}{\delta \hat{K}}\right), \\
\left(\hat{K} \mathcal{M}^{-1}\right) \cdot\left(\left(\hat{K} \mathcal{M}^{-1}\right) \cdot\left(\wedge \mathcal{M} \frac{\delta}{\delta \hat{K}}\right)\right)=\hat{K} \cdot\left(\hat{K} \cdot\left(\wedge \frac{\delta}{\delta \hat{K}}\right)\right),
\end{aligned}
$$

where $\mathcal{F}$ is a part of the kinetic operator $\mathcal{K}$, representing only the spin flip processes. Note that the term $\left(\hat{K} \mathcal{M}^{-1}\right) \cdot\left(\left(\hat{K} \mathcal{M}^{-1}\right) \cdot(\wedge \psi)\right)$ does not completely vanish in the dual theory in contrast to the corresponding identity in the original spin formulation [6]. These identities enable us to rewrite the Hamiltonian as

$$
\mathcal{H}=-\hat{K} \cdot\left(\mathcal{F} \frac{\delta}{\delta \hat{K}}\right)-\hat{K} \cdot\left(\frac{\delta}{\delta \hat{K}} \vee \frac{\delta}{\delta \hat{K}}\right)-\frac{1}{N^{2}} \hat{K} \cdot\left(\hat{K} \cdot\left(\wedge \frac{\delta}{\delta \hat{K}}\right)\right) .
$$

Justification of the identities $(4.10) \sim(4.12)$ :

We now try to establish the above identities.

(1) Kinetic term

In order to make the expression for the kinetic operator $\mathcal{F}$ explicit, we first consider the spin flip process in the continuum theory. For the universal parts of the disk and cylinder amplitudes, the string fields with a microscopic domain containing a single flipped spin are obtained from the string fields without any microscopic domain by the following rule (for derivation see Appendix C): 


$$
\begin{aligned}
-\partial_{\xi} \hat{V}_{2}(\xi)= & -\hat{\oint} \frac{d \eta}{2 \pi i} \partial_{\xi} \hat{V}^{(2)}(\xi, \eta), \\
\hat{V}_{2}^{(3)}\left(\xi_{1}, \xi_{2}, \xi_{3} ;\right)= & \hat{\oint} \frac{d \eta}{2 \pi i} \hat{V}^{(4)}\left(\xi_{1}, \xi_{2}, \xi_{3}, \eta\right), \\
& \cdots, \\
\hat{V}_{Y}^{\mathrm{cyl}(1)}(\xi)= & \hat{\oint} \frac{d \eta}{2 \pi i} \hat{V}_{1 \mid 1}^{\mathrm{cyl}}(\xi \mid \eta), \\
\hat{V}_{2 \mid Y}^{\mathrm{cyl}}\left(\xi_{1} ; \xi_{2}\right)= & \oint \frac{d \eta}{2 \pi i} \hat{V}_{Y}^{\mathrm{cyl}(3)}\left(\xi_{1}, \eta, \xi_{2}\right),
\end{aligned}
$$

where the domain corresponding to the variable $\eta$ has been shrunk into the microscopic domain by integration. The integral symbol $\hat{\oint} \frac{d \eta}{2 \pi i}$ is used in the sense of the integral with respect to the variable $y$ of the continuum theory $\left(\eta=\xi_{*}(1+a y)\right)$

$$
\hat{\oint} \frac{d \eta}{2 \pi i}=\xi_{*} a \int_{C} \frac{d y}{2 \pi i}
$$

where the contour $C$ encircles around the negative real axis and the singularities of the left half plane, and $\xi_{*}=\frac{1}{5} \hat{s} c^{-1 / 2}$. Here and in what follows $a$ is the lattice spacing. Note a slight difference from the original Ising theory in the point that there is no finite renormalization factor denoted by $s^{-1}$ in [6].

As already emphasized in [6], these relations are natural since the spin flip process occurs locally with respect to domains; in (4.14), (4.15), (4.16) and (4.17) only the $\eta$-domain is concerned and the other domains do not change at all. Because of the locality, we can reasonably expect that the relations such as $(4.14) \sim(4.17)$ should hold for any amplitudes with an arbitrary number of handles with generic spin configurations, although a completely general proof for this cannot be given at present. By assuming this, we can rewrite the spin flip process in $\left(\mathcal{K}\left(\mathcal{M} \frac{\delta}{\delta \hat{K}}+\psi\right)\right)_{X}(\xi)$ as,

$$
\begin{aligned}
- & \partial_{\xi} \oint \frac{d \eta}{2 \pi i}\left[\left(\mathcal{M} \frac{\delta}{\delta \hat{K}}\right)_{2}(\xi, \eta)+\psi_{2}(\xi, \eta)\right] \\
= & -\oint \frac{d \eta}{2 \pi i} \partial_{\xi} \frac{\delta}{\delta \hat{K}_{2}(\xi, \eta)}-\partial_{\xi}\left(\left(\frac{1}{\hat{g}}\left((1-c-\hat{g} \xi) \xi-2 \psi_{X}(\xi)\right)\right) \frac{\delta}{\delta \hat{K}_{X}(\xi)}\right) \\
& -\frac{1}{\hat{g}} \partial_{\xi}\left[\hat{g} \xi+\left((1-c-\hat{g} \xi) \xi-\psi_{X}(\xi)\right) \psi_{X}(\xi)\right],
\end{aligned}
$$

where the first term is the universal part, and the others are the non-universal parts. Similarly, we can derive the expressions for the spin-flip processes in $\left(\mathcal{K}\left(\mathcal{M} \frac{\delta}{\delta \hat{K}}+\psi\right)\right)_{2}\left(\xi_{1}, \xi_{2}\right)$. However, it must be noted that the cylinder amplitude $V_{Y}^{\mathrm{cyl}}{ }^{(1)}(\xi)$ has a non-universal cnumber term as shown in (B13) in Appendix B. In this case, a special care must be exercised in rewriting $\left(\mathcal{K}\left(\mathcal{M} \frac{\delta}{\delta \hat{K}}+\psi\right)\right)_{1}\left(\xi_{1}\right)$. Namely, from (4.7) and (B13), we can obtain the following relations:

$$
\begin{aligned}
& \left.\frac{\delta}{\delta \hat{K}_{1}(\xi)} \oint \frac{d \eta}{2 \pi i}\left[\left(\mathcal{M} \frac{\delta}{\delta \hat{K}}\right)_{1}(\eta)+\psi_{1}(\eta)\right] \hat{Z}[\hat{K}]\right|_{\hat{K}=0}=\left\langle\hat{\Psi}_{1}(\xi) \oint \frac{d \eta}{2 \pi i} \Psi_{1}(\eta)\right\rangle \\
& =\frac{1}{N^{2}} \frac{1}{\sqrt{5 c}}+\left\langle\hat{\Psi}_{1}(\xi) \oint \frac{d \eta}{2 \pi i} \hat{\Psi}_{1}(\eta)\right\rangle=\frac{1}{N^{2}} \frac{1}{\sqrt{5 c}}+\left.\frac{\delta}{\delta \hat{K}_{1}(\xi)} \hat{\oint} \frac{d \eta}{2 \pi i} \frac{\delta}{\delta \hat{K}_{1}(\eta)} \hat{Z}[\hat{K}]\right|_{\hat{K}=0},
\end{aligned}
$$


and therefore the expression requires a slight modification as follows:

$$
\oint \frac{d \eta}{2 \pi i}\left[\left(\mathcal{M} \frac{\delta}{\delta \hat{K}}\right)_{1}(\eta)+\psi_{1}(\eta)\right]=\int \frac{d \eta}{2 \pi i} \hat{K}_{1}(\eta) \frac{1}{N^{2}} \frac{1}{\sqrt{5 c}}+\hat{\oint} \frac{d \eta}{2 \pi i} \frac{\delta}{\delta \hat{K}_{1}(\eta)} .
$$

The first term in (4.21) leads to the non-universal c-number term $\frac{1}{\sqrt{5 c}}$ in the cylinder am-

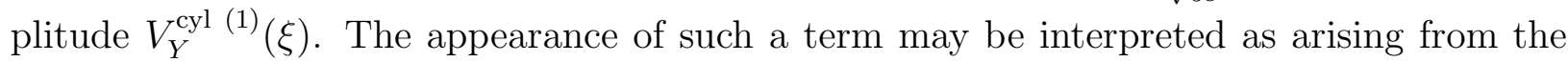
difference between the two methods of regularizations for the $\eta$-integral, namely, the lattice regularization (matrix model) and the Beta-function regularization. For higher components, we expect that there is no such modification coming from the terms containing $\hat{K}_{n}(n \geq 2)$, because $V_{Y}^{\text {cyl (3) }}$ and $V_{Y}^{\text {cyl (5) }}$ have no non-universal c-number terms and the higher cylinder amplitudes scale with negative powers of $a$, according to the results of the cylinder amplitudes $(\overline{B 16})$ and $(\bar{B} 18)$ in Appendix B.

Concerning the third term in the l.h.s. of (4.10), $(\wedge \psi)_{I, J}$ vanishes, as we will show below, except for the $I=J=1$ component:

$$
(\wedge \psi)_{I, J}\left(\xi_{1}, \cdots, \xi_{I} ; \xi_{1}^{\prime}, \cdots, \xi_{J}^{\prime}\right)= \begin{cases}\frac{1}{3}\left(\hat{g}\left(\xi_{1}+\xi_{1}^{\prime}\right)+5 c-1\right) & I=J=1 \\ 0 & \text { otherwise }\end{cases}
$$

Using these results, we arrive at Eq. (4.10) with

$$
\begin{aligned}
\hat{K} \cdot\left(\mathcal{F} \frac{\delta}{\delta \hat{K}}\right)= & \int \frac{d \xi}{2 \pi i} \hat{K}_{X}(\xi) \hat{g}\left(-\partial_{\xi}\right) \hat{\oint} \frac{d \eta}{2 \pi i} \frac{\delta}{\delta \hat{K}_{2}(\xi, \eta)} \\
& +\int \frac{d \xi_{1}}{2 \pi i} \hat{K}_{1}\left(\xi_{1}\right) \hat{g} \oint \frac{d \eta}{2 \pi i} \frac{\delta}{\delta \hat{K}_{3}\left(\xi_{1}, \eta, \xi_{1}\right)}+\cdots \\
& +\int \frac{d \xi_{1}}{2 \pi i} \frac{d \xi_{2}}{2 \pi i} \hat{K}_{2}\left(\xi_{1}, \xi_{2}\right) \hat{g} \oint \frac{d \eta}{2 \pi i}\left(\frac{\delta}{\delta \hat{K}_{4}\left(\xi_{1}, \eta, \xi_{1}, \xi_{2}\right)}+\frac{\delta}{\delta \hat{K}_{4}\left(\xi_{1}, \xi_{2}, \eta, \xi_{2}\right)}\right)+\cdots \\
& +\cdots,
\end{aligned}
$$

where the ellipsis stands for the subleading terms and the terms containing the higher components $\hat{K}_{n}(n \geq 3)$.

It is noted that the tadpole term is canceled with a contribution of the same form from the kinetic term, as in the original Ising theory [6], and that $\psi$-dependent terms are absent because their contributions from kinetic and splitting terms cancel each other. In particular the leading contribution of $(\wedge \psi)_{1,1}$ is precisely canceled with the non-universal c-number term of (4.21). Although we do not elaborate further on determining the explicit continuum limits for higher components, it is natural, because of the local nature of the spin-flip processes, to suppose that the above expression already indicates the generic structure of the kinetic term, namely the flipping of a single spin on a loop with general spin configurations, and the absence of the tadpole terms and the terms without spin flipping.

\section{(2) Interaction term}

Next, we consider the splitting and merging processes, Eqs. (4.11) and (4.12). In the next section, we will argue the validity of the following important relations, showing the commutativity of the splitting and merging processes with the operator mixing, 


$$
\begin{gathered}
\left(\left(\mathcal{M} \frac{\delta}{\delta \hat{K}}\right) \vee\left(\mathcal{M} \frac{\delta}{\delta \hat{K}}\right)\right)_{I}=\left(\mathcal{M}\left(\frac{\delta}{\delta \hat{K}} \vee \frac{\delta}{\delta \hat{K}}\right)\right)_{I} \\
\left(\wedge\left(\mathcal{M} \frac{\delta}{\delta \hat{K}}\right)\right)_{I, J}=\sum_{K, L} \mathcal{M}_{I K} \mathcal{M}_{J L}\left(\wedge \frac{\delta}{\delta \hat{K}}\right)_{K, L},
\end{gathered}
$$

for completely general case, provided the mixing obeys some rules which can be explicitly confirmed for several lower nontrivial components. H This commutativity property ensures that the general structure of the splitting and the merging in the continuum limit are essentially the same as in the lattice theory.

(3) C-number term

Finally, let us turn to (4.22). We can check its validity for the $\operatorname{components}(I, J)=$ $(X, X),(X, 2),(X, 2 k+1),(1,1),(1,3),(1,2 k),(2,2),(2,2 k+1)(k$ is integer) by direct calculation. For higher components, we give a general proof of (4.22) in the same way as [6]. First we show that $\psi_{2 k}$ must be polynomial for every $k$. For this purpose, we start with the Schwinger-Dyson equations and derive the dual version of Staudacher's recursion equations [12]. They relate $V^{(k)}$ to the amplitudes $V^{(l)}(l<k)$ as

$$
\begin{aligned}
& V^{(2 k)}\left(\xi_{1}, \cdots, \xi_{2 k}\right)=\frac{1}{1+c-\hat{g}\left(\xi_{1}+\xi_{2}\right)} \\
& \times\left\{V\left(\xi_{1}\right)\left(-D_{\xi}\left(\xi_{2}, \xi_{2 k}\right)\right) V^{(2 k-2)}\left(\xi_{1}, \xi_{3}, \cdots, \xi_{2 k-1}\right)+V\left(\xi_{2}\right)\left(-D_{\xi}\left(\xi_{1}, \xi_{3}\right)\right) V^{(2 k-2)}\left(\xi, \xi_{4}, \cdots, \xi_{2 k}\right)\right. \\
& +\sum_{l=2}^{k-1}\left[D_{\xi}\left(\xi_{2}, \xi_{2 l}\right) V^{(2 l-2)}\left(\xi, \xi_{3}, \cdots, \xi_{2 l-1}\right)\right]\left[D_{\xi}\left(\xi_{1}, \xi_{2 l+1}\right) V^{(2 k-2 l)}\left(\xi, \xi_{2 l+2}, \cdots, \xi_{2 k}\right)\right] \\
& \left.-\hat{g} V_{2}^{(2 k-1)}\left(\xi_{2}, \cdots, \xi_{2 k} ;\right)-\hat{g} V_{2}^{(2 k-1)}\left(\xi_{3}, \cdots, \xi_{2 k}, \xi_{1} ;\right)\right\}, \\
& \hat{g} V_{2}^{(2 k-1)}\left(\xi_{2}, \cdots, \xi_{2 k} ;\right) \\
& =(-1)^{k+1} \hat{g} V_{2}\left(\xi_{k+1}\right)+\sum_{p=0}^{k-2}(-1)^{k+p+1} D_{\xi}\left(\xi_{k-p}, \xi_{k+p+2}\right) \\
& \times\left[\left((1-c) \xi-\hat{g} \xi^{2}-V\left(\xi_{k-p}\right)-V\left(\xi_{k+p+2}\right)\right) V^{(2 p+2)}\left(\xi, \xi_{k-p+1}, \cdots, \xi_{k+p+1}\right)\right] \\
& +\sum_{p=0}^{k-2} \sum_{l=1}^{p}(-1)^{k+p+1}\left[D_{\xi}\left(\xi_{k-p}, \xi_{k-p+2 l}\right) V^{(2 l)}\left(\xi, \xi_{k-p+1}, \cdots, \xi_{k-p+2 l-1}\right)\right] \\
& \left.\times\left[D_{\xi}\left(\xi_{k-p+2 l}, \xi_{k+p+2}\right) V^{(2 p-2 l+2)}\left(\xi, \xi_{k-p+2 l+1}, \cdots, \xi_{k+p+1}\right)\right]\right\},
\end{aligned}
$$

and $\hat{g} V_{2}^{(2 k-1)}\left(\xi_{3}, \cdots, \xi_{2 k}, \xi_{1} ;\right)$ has a similar form. Suppose that $\psi_{2 k}$ 's are polynomials up to some $k$. Then using above equations we see that the part of the numerator for $V^{(2 k)}$ consisting only of $\psi$ is a polynomial, because in general the combinatorial derivative of a

\footnotetext{
4 The proof of the next section is applicable both for the original and dual theories, generalizing the results for the original theory given in the previous work [6].
} 
polynomial is also a polynomial and c-number function $\psi$ in $V_{2}\left(\xi_{k+1}\right)$ is a polynomial. The denominator, on the other hand, behaves in the scaling limit as

$$
1+c-\hat{g}\left(\xi_{1}+\xi_{2}\right)=-a c \hat{s}\left(y_{1}+y_{2}\right)+O\left(a^{2}\right) .
$$

Thus, by using the scaled variables $\xi_{i}=\xi_{*}\left(1+a y_{i}\right), \psi_{2 k}$ can be written as

$$
\psi_{2 k}=\frac{\text { Polynomial of }\left(y_{1}, y_{2}, \cdots, y_{2 k}\right)}{y_{1}+y_{2}} .
$$

However, from the cyclic symmetry with respect to $\xi_{i}$ 's in $V^{(2 k)}\left(\xi_{1}, \xi_{2}, \cdots, \xi_{2 k}\right)$, the denominator $y_{1}+y_{2}$ must be cancelled with the numerator, and thus $\psi_{2 k}$ should have the form:

$$
\psi_{2 k}=\text { Polynomial of }\left(y_{1}, y_{2}, \cdots, y_{2 k}\right) \text {, }
$$

where the polynomial has the same symmetry as $V^{(2 k)}$. Thus, by induction, the $\psi_{2 k}$ must be a polynomial for general $k$.

Now from the scaling behavior of the universal part $\hat{V}^{(2 k)}$

$$
\hat{V}^{(2 k)}\left(\xi_{1}, \cdots, \xi_{2 k}\right)=\left\langle\hat{\Psi}_{2 k}\left(\xi_{1}, \cdots, \xi_{2 k}\right)\right\rangle_{0}=a^{\frac{7}{3}-\frac{2}{3} k} v^{(2 k)}\left(y_{1}, y_{2}, \cdots, y_{2 k}\right)
$$

derived in Appendix B, we expect that the relevant part of $\psi_{2 k}$ takes the form

$$
\psi_{2 k}= \begin{cases}\text { const. } & k=3 \\ 0 & k \geq 4\end{cases}
$$

Since every component of $\wedge \psi$ contains the derivative or the combinatorial derivative, Eq. (4.28) leads to (4.22).

We are now ready to take the continuum limit of the stochastic Hamiltonian (4.13). The scaling property of the string fields presented in Appendix B enables us to introduce the variables in the continuum theory as follows:

$$
\begin{aligned}
& \hat{g}=\hat{g}_{*}\left(1-a^{2} \frac{\hat{s}^{2}}{10} \hat{T}\right), \quad \xi=\xi_{*}(1+a y), \quad \frac{1}{N}=a^{7 / 3} g_{\text {st }}, \\
& \frac{\delta}{\delta \hat{K}_{X}(\xi)}= a^{4 / 3} \xi_{*}^{-1} \frac{\delta}{\delta \tilde{K}_{X}(y)}, \quad \hat{K}_{X}(\xi)=a^{-7 / 3} \tilde{K}_{X}(y), \\
& \frac{\delta}{\delta \hat{K}_{n}\left(\xi_{1}, \cdots, \xi_{n}\right)}= a^{(7-n) / 3} \xi_{*}^{-n} \frac{\delta}{\delta \tilde{K}_{n}\left(y_{1}, \cdots, y_{n}\right)}, \\
& \hat{K}_{n}\left(\xi_{1}, \cdots, \xi_{n}\right)=a^{-(7+2 n) / 3} \tilde{K}_{n}\left(y_{1}, \cdots, y_{n}\right), \\
&(n=1,2,3, \cdots) .
\end{aligned}
$$

In the limit $a \rightarrow 0$, the leading contributions in (4.13) start with $O\left(a^{1 / 3}\right)$, which come from the $X_{\alpha}$-derivatives in (3.4). On the other hand, the contributions from the $Y_{\alpha}$-derivatives are subleading and do not survive in the continuum limit. After the finite rescaling

$$
\tilde{K}_{I} \rightarrow\left(\xi_{*} \hat{g}_{*}\right)^{-1} \tilde{K}_{I}, \quad \frac{\delta}{\delta \tilde{K}_{I}} \rightarrow \xi_{*} \hat{g}_{*} \frac{\delta}{\delta \tilde{K}_{I}}, \quad g_{\mathrm{st}}^{2} \rightarrow\left(\xi_{*} \hat{g}_{*}\right)^{2} g_{\mathrm{st}}^{2}
$$


and absorbing the overall factor $a^{1 / 3} \xi_{*}^{-1} \hat{g}_{*}$ into the renormalization of the fictitious time, we have the final result for the continuum stochastic Hamiltonian:

$$
\mathcal{H}_{D}=-\tilde{K} \cdot\left(\mathcal{F} \frac{\delta}{\delta \tilde{K}}\right)-\tilde{K} \cdot\left(\frac{\delta}{\delta \tilde{K}} \vee \frac{\delta}{\delta \tilde{K}}\right)-g_{\text {st }}^{2} \tilde{K} \cdot\left(\tilde{K} \cdot\left(\wedge \frac{\delta}{\delta \tilde{K}}\right)\right)
$$

where the inner product is defined by

$$
f \cdot g \equiv \int_{-i \infty}^{i \infty} \frac{d y}{2 \pi i} f_{X}(y) g_{X}(y)+\sum_{n=1}^{\infty} \int_{-i \infty}^{i \infty} \prod_{i=1}^{n} \frac{d y_{i}}{2 \pi i} f_{n}\left(y_{1}, \cdots, y_{n}\right) g_{n}\left(y_{1}, \cdots, y_{n}\right)
$$

Each term in (4.30) has the following structure. The first term is the kinetic term consisting only of the spin-flip processes:

$$
\begin{aligned}
\left(\mathcal{F} \frac{\delta}{\delta \tilde{K}}\right)_{X}(y)= & -\partial_{y} \int_{C} \frac{d x}{2 \pi i} \frac{\delta}{\delta \tilde{K}_{2}(y, x)} \\
\left(\mathcal{F} \frac{\delta}{\delta \tilde{K}}\right)_{1}\left(y_{1}\right)= & \int_{C} \frac{d x}{2 \pi i} \frac{\delta}{\delta \tilde{K}_{3}\left(y_{1}, x, y_{1}\right)}, \\
\left(\mathcal{F} \frac{\delta}{\delta \tilde{K}}\right)_{2}\left(y_{1}, y_{2}\right)= & \int_{C} \frac{d x}{2 \pi i}\left[\frac{\delta}{\delta \tilde{K}_{4}\left(y_{1}, x, y_{1}, y_{2}\right)}+\frac{\delta}{\delta \tilde{K}_{4}\left(y_{1}, y_{2}, x, y_{2}\right)}\right] \\
& \cdots, \\
\left(\mathcal{F} \frac{\delta}{\delta \tilde{K}}\right)_{n}\left(y_{1}, \cdots, y_{n}\right)= & \sum_{j=1}^{n} \int_{C} \frac{d x}{2 \pi i} \frac{\delta}{\delta \tilde{K}_{n+2}\left(y_{1}, \cdots, y_{j}, x, y_{j}, \cdots, y_{n}\right)}, \\
& \cdots
\end{aligned}
$$

The second and third terms represent the splitting and merging processes, respectively. Due to the commutativity of these processes with the operator mixing, they have the same structure as the contributions from the $X_{\alpha}$ - derivatives in the lattice theory. The components of the splitting term are given as

$$
\begin{gathered}
\left(\frac{\delta}{\delta \tilde{K}} \vee \frac{\delta}{\delta \tilde{K}}\right)_{X}(y)=-\partial_{y} \frac{\delta^{2}}{\delta \tilde{K}_{X}(y)^{2}}, \\
\left(\frac{\delta}{\delta \tilde{K}} \vee \frac{\delta}{\delta \tilde{K}}\right)_{1}\left(y_{1}\right)=-2 \frac{\delta}{\delta \tilde{K}_{X}\left(y_{1}\right)} \partial_{y_{1}} \frac{\delta}{\delta \tilde{K}_{1}\left(y_{1}\right)}, \\
\left(\frac{\delta}{\delta \tilde{K}} \vee \frac{\delta}{\delta \tilde{K}}\right)_{2}\left(y_{1}, y_{2}\right)=-2 \sum_{j=1}^{2} \frac{\delta}{\delta \tilde{K}_{X}\left(y_{j}\right)} \partial_{y_{j}} \frac{\delta}{\delta \tilde{K}_{2}\left(y_{1}, y_{2}\right)}+2\left(D_{z}\left(y_{1}, y_{2}\right) \frac{\delta}{\delta \tilde{K}_{1}(z)}\right)^{2}, \\
\left(\frac{\delta}{\delta \tilde{K}} \vee \frac{\delta}{\delta \tilde{K}}\right)_{n}\left(y_{1}, \cdots, y_{n}\right)=-2 \sum_{j=1}^{n} \frac{\delta}{\delta \tilde{K}_{X}\left(y_{j}\right)} \partial_{y_{j}} \frac{\delta}{\delta \tilde{K}_{n}\left(y_{1}, \cdots, y_{n}\right)} \\
+2 \sum_{k<l} D_{z}\left(y_{k}, y_{l}\right) \frac{\delta}{\delta \tilde{K}_{l-k}\left(z, y_{k+1}, \cdots, y_{l-1}\right)} D_{w}\left(y_{k}, y_{l}\right) \frac{\delta \tilde{K}_{n-l+k}\left(y_{1}, \cdots, y_{k-1}, w, y_{l+1}, \cdots, y_{n}\right)}{\delta \tilde{K}, \cdots}
\end{gathered}
$$


Also, the components of the merging term are

$$
\begin{gathered}
\left(\wedge \frac{\delta}{\delta \tilde{K}}\right)_{X, X}\left(y ; y^{\prime}\right)=-\partial_{y} \partial_{y^{\prime}} D_{z}\left(y, y^{\prime}\right) \frac{\delta}{\delta \tilde{K}_{X}(z)}, \\
\left(\wedge \frac{\delta}{\delta \tilde{K}}\right)_{n, X}\left(y_{1}, \cdots, y_{n} ; y^{\prime}\right)=\left(\wedge \frac{\delta}{\delta \tilde{K}}\right)_{X, n}\left(y^{\prime} ; y_{1}, \cdots, y_{n}\right) \\
=-\partial_{y^{\prime}} \sum_{j=1}^{n} \partial_{y_{j}} D_{z}\left(y_{j}, y^{\prime}\right) \frac{\delta}{\delta \tilde{K}_{n}\left(y_{1}, \cdots, y_{j-1}, z, y_{j+1}, \cdots, y_{n}\right)} \quad(n \geq 1), \\
\left(\wedge \frac{\delta}{\delta \tilde{K}}\right)_{n, 1}\left(y_{1}, \cdots, y_{n} ; y_{1}^{\prime}\right)=\left(\wedge \frac{\delta}{\delta \tilde{K}_{1, n}}\right)_{1, n}\left(y_{1}^{\prime} ; y_{1}, \cdots, y_{n}\right) \\
=\sum_{j=1}^{n} D_{z}\left(y_{j}, y_{1}^{\prime}\right) D_{w}\left(y_{j}, y_{1}^{\prime}\right) \frac{\delta}{\delta \tilde{K}_{n+1}\left(y_{1}, \cdots, y_{j-1}, z, w, y_{j+1}, \cdots, y_{n}\right)} \quad(n \geq 2), \\
\left(\wedge \frac{\delta}{\delta \tilde{K}}\right)_{n, m}\left(y_{1} ; y_{1}^{\prime}\right)=D_{z}\left(y_{1}, y_{1}^{\prime}\right) D_{w}\left(y_{1}, y_{1}^{\prime}\right) \frac{\left.\delta, y_{n} ; y_{1}^{\prime}, \cdots, y_{m}^{\prime}\right)=\sum_{j=1}^{n} \sum_{k=1}^{m} D_{z}\left(y_{j}, y_{k}^{\prime}\right) D_{w}\left(y_{j}, y_{k}^{\prime}\right)}{\delta}, \\
\times \frac{\delta \tilde{K}_{n+m}\left(y_{1}, \cdots, y_{j-1}, z, y_{k+1}^{\prime}, \cdots, y_{m}^{\prime}, y_{1}^{\prime}, \cdots, y_{k-1}^{\prime}, w, y_{j+1}, \cdots, y_{n}\right)}{(n, m \geq 2) .}
\end{gathered}
$$

As we have promised earlier, the terms of the Hamiltonian arising from the $Y_{\alpha}$-derivatives do not contribute in the continuum limit. Thus it should be noted that the symbols $\vee$ and $\wedge$ used here do not represent completely identical objects with $\vee$ and $\wedge$ in eq. (4.13), because the latter contains terms vanishing in the continuum limit which originate from the $Y_{\alpha}$-derivatives in Eq. (3.4).

In this Hamiltonian, the interactions including the string fields with odd domains survive after the continuum limit. This is important for discussing the duality symmetry. Remember that the string fields with even domains can be interpreted in terms of dual spins, while those with odd domains cannot be. If we allow only the strings with even domains in the initial state and consider its time evolution by this Hamiltonian within the tree approximation, the strings with odd domains never contribute, because the vacuum expectation value of a single string field with odd domains vanishes owing to the $\boldsymbol{Z}_{2}$-symmetry. In general, however, the strings with odd domains necessarily contribute in the intermediate states, since there exists such a process that a string with even domains splits into two strings with odd domains and they merge to a single string again. It represents the excitation of odd $Y$-loops along the non-trivial cycles of the handles. In section VI, we will further examine the correspondence between the original and dual theories beyond the level of the disk and cylinder amplitudes presented in Appendix B. 


\section{ALGEBRA OF SPLITTING AND MERGING INTERACTIONS}

We saw that the commutativity of the mixing matrix with splitting and merging processes plays a crucial role in obtaining the continuum Hamiltonian in both the original and the dual models. In view of its potential importance for the development of string field theories, we devote the present section to its derivation and further elucidation.

The commutativity was previously confirmed explicitly in several simple (but nontrivial) configurations and conjectured for general configurations. Although we cannot still give a complete proof, we shall improve the situation by proposing the general form of $\mathcal{M}_{I J}$, and then proving the commutativity for generic case on the basis of this assumption.

The general forms of the mixing matrices are summarized as:

1. $\mathcal{M}$ can be expressed in terms of a matrix $\mathcal{R}$ as

$$
\mathcal{M}_{I J}=\left(e^{\alpha \mathcal{R}}\right)_{I J}
$$

where $\alpha$ is a constant whose value is $\alpha=\sqrt{10 c}$ for the original model and $\alpha=\frac{2}{\sqrt{5 c}}$ for the dual one.

2. The definition of $\mathcal{R}$.

(a) The original model:

$$
\begin{aligned}
& (\mathcal{R} f)_{A}(\zeta)=0, \quad(\mathcal{R} f)_{B}(\sigma)=0, \quad(\mathcal{R} f)_{1}(\zeta, \sigma)=f_{A}(\zeta)+f_{B}(\sigma), \\
& (\mathcal{R} f)_{n}\left(\zeta_{1}, \sigma_{1}, \cdots, \zeta_{n}, \sigma_{n}\right) \quad(n \geq 2) \\
& =-\sum_{j=1}^{n} D_{\zeta}\left(\zeta_{j}, \zeta_{j+1}\right) f_{n-1}\left(\zeta_{1}, \cdots, \sigma_{j-1}, \zeta, \sigma_{j+1}, \cdots, \sigma_{n}\right) \\
& \quad-\sum_{j=1}^{n} D_{\sigma}\left(\sigma_{j-1}, \sigma_{j}\right) f_{n-1}\left(\zeta_{1}, \cdots, \zeta_{j-1}, \sigma, \zeta_{j+1}, \cdots, \sigma_{n}\right) \\
& \left(\zeta_{0} \equiv \zeta_{n}, \zeta_{n+1} \equiv \zeta_{1}, \sigma_{0} \equiv \sigma_{n}, \sigma_{n+1} \equiv \sigma_{1}\right) .
\end{aligned}
$$

(b) The dual model:

$$
\begin{aligned}
& (\mathcal{R} f)_{X}(\xi)=0, \quad(\mathcal{R} f)_{1}\left(\xi_{1}\right)=0, \quad(\mathcal{R} f)_{2}\left(\xi_{1}, \xi_{2}\right)=f_{X}\left(\xi_{1}\right)+f_{X}\left(\xi_{2}\right), \\
& (\mathcal{R} f)_{n}\left(\xi_{1}, \cdots, \xi_{n}\right) \quad(n \geq 3) \\
& \quad=-\sum_{j=1}^{n} D_{z}\left(\xi_{j-1}, \xi_{j+1}\right) f_{n-2}\left(\xi_{1}, \cdots, \xi_{j-2}, z, \xi_{j+2}, \cdots, \xi_{n}\right) \\
& \quad\left(\xi_{0} \equiv \xi_{n}, \xi_{n+1} \equiv \xi_{1}\right) .
\end{aligned}
$$

The validity of these rules is explicitly confirmed for the original model up to the $I=$ $A, B, 1,2$ components of $\mathcal{M}_{I J}$ as given in Eq. (99) of [6], and for the dual model up to the $I=X, 1,2,3,4,5$ components of $\mathcal{M}_{I J}$ as given in Eqs. (4.2) and (B18).

The physical meaning of the operator $\mathcal{R}$ is reducing one domain of spins. Reduction of one domain in generic spin configurations causes merging of two domains which have 
been separated by the reduced domain. This explains the appearance of the combinatorial derivatives in $\mathcal{R}$.

Now assuming the above general structure (5.1) for $\mathcal{M}_{I J}$, the proof of the commutativity Eqs. (4.24) and (4.25) is reduced to establish the following equations (5.4) and (5.5), respectively:

$$
\begin{array}{r}
(\mathcal{R}(f \vee g))_{I}=((\mathcal{R} f) \vee g)_{I}+(f \vee(\mathcal{R} g))_{I} \\
(\wedge(\mathcal{R} f))_{I, J}=\sum_{K} \mathcal{R}_{I K}(\wedge f)_{K, J}+\sum_{L} \mathcal{R}_{J L}(\wedge f)_{I, L},
\end{array}
$$

where we introduced two independent arbitrary vectors $f_{I}$ and $g_{I}$ to emphasize that the above equations should be valid irrespectively of the particular structure of $\left(\frac{\delta}{\delta \hat{J}}\right)_{I}$ or $\left(\frac{\delta}{\delta \hat{K}}\right)_{I}$. The relations (5.4) and (5.5) say that $\mathcal{R}$ acts like a derivation on the string fields with respect to the rule of their multiplications defined by the merging-splitting interactions. Note also the possibility of interpreting the operator $\mathcal{R}$ as a conserved charge on the world sheet.

The definitions of $\vee$ and $\wedge$ for lower components in the original model are given in (95) and (96) of [6]. For attempting the proof of commutativity, we need more precise definitions for general configurations.

$$
\begin{aligned}
& (f \vee g)_{A}(\zeta)=-f_{A}(\zeta) \partial_{\zeta} g_{A}(\zeta)-g_{A}(\zeta) \partial_{\zeta} f_{A}(\zeta) \\
& (f \vee g)_{B}(\sigma)=-f_{B}(\sigma) \partial_{\sigma} g_{B}(\sigma)-g_{B}(\sigma) \partial_{\sigma} f_{B}(\sigma) \\
& (f \vee g)_{n}\left(\zeta_{1}, \sigma_{1}, \cdots, \zeta_{n}, \sigma_{n}\right) \\
& =-\sum_{k=1}^{n} f_{A}\left(\zeta_{k}\right) \partial_{\zeta_{k}} g_{n}\left(\zeta_{1}, \sigma_{1}, \cdots, \zeta_{n}, \sigma_{n}\right)-\sum_{k=1}^{n} f_{B}\left(\sigma_{k}\right) \partial_{\sigma_{k}} g_{n}\left(\zeta_{1}, \sigma_{1}, \cdots, \zeta_{n}, \sigma_{n}\right) \\
& \quad+\sum_{k<l} D_{\zeta}\left(\zeta_{k}, \zeta_{l}\right) f_{l-k}\left(\zeta, \sigma_{k}, \cdots, \zeta_{l-1}, \sigma_{l-1}\right) D_{\zeta^{\prime}}\left(\zeta_{k}, \zeta_{l}\right) g_{n-l+k}\left(\zeta_{1}, \cdots, \zeta_{k-1}, \sigma_{k-1}, \zeta^{\prime}, \sigma_{l}, \cdots, \sigma_{n}\right) \\
& \quad+\sum_{k<l} D_{\sigma}\left(\sigma_{k}, \sigma_{l}\right) f_{l-k}\left(\zeta_{k+1}, \sigma_{k+1}, \cdots, \zeta_{l}, \sigma\right) D_{\sigma^{\prime}}\left(\sigma_{k}, \sigma_{l}\right) g_{n-l+k}\left(\zeta_{1}, \cdots, \zeta_{k}, \sigma^{\prime}, \zeta_{l+1}, \sigma_{l+1}, \cdots, \sigma_{n}\right) \\
& \quad-\sum_{k=1}^{n} g_{A}\left(\zeta_{k}\right) \partial_{\zeta_{k}} f_{n}\left(\zeta_{1}, \sigma_{1}, \cdots, \zeta_{n}, \sigma_{n}\right)-\sum_{k=1}^{n} g_{B}\left(\sigma_{k}\right) \partial_{\sigma_{k}} f_{n}\left(\zeta_{1}, \sigma_{1}, \cdots, \zeta_{n}, \sigma_{n}\right) \\
& \quad+\sum_{k<l} D_{\zeta}\left(\zeta_{k}, \zeta_{l}\right) g_{l-k}\left(\zeta, \sigma_{k}, \cdots, \zeta_{l-1}, \sigma_{l-1}\right) D_{\zeta^{\prime}}\left(\zeta_{k}, \zeta_{l}\right) f_{n-l+k}\left(\zeta_{1}, \cdots, \zeta_{k-1}, \sigma_{k-1}, \zeta^{\prime}, \sigma_{l}, \cdots, \sigma_{n}\right) \\
& \quad+\sum_{k<l} D_{\sigma}\left(\sigma_{k}, \sigma_{l}\right) g_{l-k}\left(\zeta_{k+1}, \sigma_{k+1}, \cdots, \zeta_{l}, \sigma\right) D_{\sigma^{\prime}}\left(\sigma_{k}, \sigma_{l}\right) f_{n-l+k}\left(\zeta_{1}, \cdots, \zeta_{k}, \sigma^{\prime}, \zeta_{l+1}, \sigma_{l+1}, \cdots, \sigma_{n}\right),
\end{aligned}
$$

$$
\begin{aligned}
& (\wedge f)_{A, A}\left(\zeta ; \zeta^{\prime}\right)=-\partial_{\zeta} \partial_{\zeta^{\prime}} D_{z}\left(\zeta, \zeta^{\prime}\right) f_{A}(z), \\
& (\wedge f)_{A, B}(\zeta ; \sigma)=(\wedge f)_{B, A}(\sigma ; \zeta)=0 \\
& (\wedge f)_{B, B}\left(\sigma ; \sigma^{\prime}\right)=-\partial_{\sigma} \partial_{\sigma^{\prime}} D_{s}\left(\sigma, \sigma^{\prime}\right) f_{B}(s), \\
& (\wedge f)_{n, A}\left(\zeta_{1}, \sigma_{1}, \cdots, \zeta_{n}, \sigma_{n} ; \zeta^{\prime}\right)=(\wedge f)_{A, n}\left(\zeta^{\prime} ; \zeta_{1}, \sigma_{1}, \cdots, \zeta_{n}, \sigma_{n}\right) \\
& \quad=-\partial_{\zeta^{\prime}} \sum_{k=1}^{n} \partial_{\zeta_{k}} D_{z}\left(\zeta_{k}, \zeta^{\prime}\right) f_{n}\left(\zeta_{1}, \cdots, \zeta_{k-1}, \sigma_{k-1}, z, \sigma_{k}, \cdots, \sigma_{n}\right), \\
& (\wedge f)_{n, B}\left(\zeta_{1}, \sigma_{1}, \cdots, \zeta_{n}, \sigma_{n} ; \sigma^{\prime}\right)=(\wedge f)_{B, n}\left(\sigma^{\prime} ; \zeta_{1}, \sigma_{1}, \cdots, \zeta_{n}, \sigma_{n}\right)
\end{aligned}
$$




$$
\begin{aligned}
& =-\partial_{\sigma^{\prime}} \sum_{k=1}^{n} \partial_{\sigma_{k}} D_{s}\left(\sigma_{k}, \sigma^{\prime}\right) f_{n}\left(\zeta_{1}, \cdots, \zeta_{k}, s, \zeta_{k+1}, \sigma_{k+1}, \cdots, \sigma_{n}\right) \\
& (\wedge f)_{n, m}\left(\zeta_{1}, \sigma_{1}, \cdots, \zeta_{n}, \sigma_{n} ; \zeta_{1}^{\prime}, \sigma_{1}^{\prime}, \cdots, \zeta_{m}^{\prime}, \sigma_{m}^{\prime}\right) \\
& =\sum_{k=1}^{n} \sum_{l=1}^{m} D_{z}\left(\zeta_{k}, \zeta_{l}^{\prime}\right) D_{w}\left(\zeta_{k}, \zeta_{l}^{\prime}\right) f_{n+m}\left(\zeta_{1}, \sigma_{1}, \cdots, \sigma_{k-1}, z, \sigma_{l}^{\prime}, \cdots, \sigma_{m}^{\prime}, \zeta_{1}^{\prime}, \cdots, \sigma_{l-1}^{\prime}, w, \sigma_{k}, \cdots, \sigma_{n}\right) \\
& +\sum_{k=1}^{n} \sum_{l=1}^{m} D_{s}\left(\sigma_{k}, \sigma_{l}^{\prime}\right) D_{t}\left(\sigma_{k}, \sigma_{l}^{\prime}\right) f_{n+m}\left(\zeta_{1}, \cdots, \zeta_{k}, s, \zeta_{l+1}^{\prime}, \cdots, \zeta_{m}^{\prime}, \sigma_{m}^{\prime} \zeta_{1}^{\prime}, \cdots, \zeta_{l}^{\prime}, t, \zeta_{k+1}, \cdots, \zeta_{n}, \sigma_{n}\right) .
\end{aligned}
$$

Note that we have slightly generalized the original definition of $\vee$ by introducing two different vectors $f_{I}$ and $g_{I}$.

For the dual model, we have already presented the definitions of $\vee$ and $\wedge$ in (3.10) and (3.11) in section III. Again, we make a slight generalization of the definition of $\vee$ as above. The prescription in this generalization is the same as above. It is sufficient to explain it using a simple example. For instance, the $I=1$ component for the dual model is defined in (3.10) as

$$
\left(\frac{\delta}{\delta K} \vee \frac{\delta}{\delta K}\right)_{1}\left(\xi_{1}\right)=-2 \frac{\delta}{\delta K_{X}\left(\xi_{1}\right)} \partial_{\xi_{1}} \frac{\delta}{\delta K_{1}\left(\xi_{1}\right)}
$$

Correspondingly, we generalize the definition as

$$
(f \vee g)_{1}\left(\xi_{1}\right)=-f_{X}\left(\xi_{1}\right) \partial_{\xi_{1}} g_{1}\left(\xi_{1}\right)-g_{X}\left(\xi_{1}\right) \partial_{\xi_{1}} f_{1}\left(\xi_{1}\right)
$$

The prescriptions for generic cases can be easily deduced from this example.

Let us now proceed to prove Eqs. (5.4) and (5.5) for both original and dual models on the basis of the rules $(5.1) \sim(5.3)$. Most parts of the proof consist of straightforward calculations after plugging all these definitions into the formulas. However, we need several non-trivial identities for some cases. The set of such identities and the cases to use them are the same for both original and dual models, signaling their universal nature.

For Eq. (5.4), we use the following two identities valid for arbitrary functions $F(\zeta), G(\zeta)$ and $H(z, w)$ :

$$
\begin{aligned}
& D_{\zeta}\left(\zeta_{1}, \zeta_{2}\right) F(\zeta) \partial_{\zeta} G(\zeta)=\sum_{k=1}^{2} F\left(\zeta_{k}\right) \partial_{\zeta_{k}} D_{\zeta}\left(\zeta_{1}, \zeta_{2}\right) G(\zeta)+D_{\zeta}\left(\zeta_{1}, \zeta_{2}\right) F(\zeta) D_{\zeta^{\prime}}\left(\zeta_{1}, \zeta_{2}\right) G\left(\zeta^{\prime}\right), \\
& D_{\zeta}\left(\zeta_{1}, \zeta_{2}\right) D_{\zeta^{\prime}}\left(\zeta, \zeta_{k}\right) F\left(\zeta^{\prime}\right) D_{\zeta^{\prime \prime}}\left(\zeta, \zeta_{k}\right) G\left(\zeta^{\prime \prime}\right) \\
& =D_{\zeta}\left(\zeta_{1}, \zeta_{k}\right) D_{\zeta^{\prime}}\left(\zeta, \zeta_{2}\right) F\left(\zeta^{\prime}\right) D_{\zeta^{\prime \prime}}\left(\zeta_{1}, \zeta_{k}\right) G\left(\zeta^{\prime \prime}\right)+D_{\zeta^{\prime}}\left(\zeta_{2}, \zeta_{k}\right) F\left(\zeta^{\prime}\right) D_{\zeta}\left(\zeta_{2}, \zeta_{k}\right) D_{\zeta^{\prime \prime}}\left(\zeta_{1}, \zeta\right) G\left(\zeta^{\prime \prime}\right)
\end{aligned}
$$

For Eq. (5.5):

$$
\begin{gathered}
D_{\zeta}\left(\zeta_{1}, \zeta_{2}\right) \partial_{\zeta} D_{z}\left(\zeta, \zeta^{\prime}\right) F(z)=\partial_{\zeta_{1}} D_{z}\left(\zeta^{\prime}, \zeta_{1}\right) D_{\zeta}\left(z, \zeta_{2}\right) F(\zeta)+\partial_{\zeta_{2}} D_{z}\left(\zeta^{\prime}, \zeta_{2}\right) D_{\zeta}\left(\zeta_{1}, z\right) F(\zeta) \\
\partial_{\zeta} \partial_{\zeta^{\prime}} D_{z}\left(\zeta, \zeta^{\prime}\right) F(z)=D_{z}\left(\zeta, \zeta^{\prime}\right) D_{w}\left(\zeta, \zeta^{\prime}\right) D_{\zeta}(z, w) F(\zeta)
\end{gathered}
$$




$$
\begin{aligned}
& D_{\zeta}\left(\zeta_{1}, \zeta_{2}\right) D_{z}\left(\zeta, \zeta^{\prime}\right) D_{w}\left(\zeta, \zeta^{\prime}\right) H(z, w) \\
& =D_{\zeta}\left(\zeta_{2}, \zeta^{\prime}\right) D_{z}\left(\zeta, \zeta_{1}\right) D_{w}\left(\zeta_{2}, \zeta^{\prime}\right) H(z, w) \\
& \quad+D_{\zeta}\left(\zeta_{1}, \zeta^{\prime}\right) D_{z}\left(\zeta_{1}, \zeta^{\prime}\right) D_{w}\left(\zeta, \zeta_{2}\right) H(z, w)
\end{aligned}
$$

The nature of these identities can be seen pictorially in Figure $1 \sim$ Figure 5. f For convenience of the reader, we have included a Table, indicating precisely where these identities should be used. Identities (5.9), (5.11) and (5.12) have already appeared in Appendix E of [6] as Eqs. (E6), (E10) and (E11), respectively. On the other hand, identities (5.10) and (5.13) are not mentioned in [6] where simpler spin configurations are dealt with.

Finally, we will explain a property of the combinatorial derivative which is useful in the proof. Obviously, the combinatorial derivative is symmetric under interchange of its arguments:

$$
D_{z}\left(\zeta_{1}, \zeta_{2}\right) F(z)=\frac{F\left(\zeta_{1}\right)}{\zeta_{1}-\zeta_{2}}+\frac{F\left(\zeta_{2}\right)}{\zeta_{2}-\zeta_{1}} .
$$

For two or three successive combinatorial derivatives, we have

$$
\begin{aligned}
& D_{z}\left(\zeta_{1}, \zeta_{2}\right) D_{z^{\prime}}\left(z, \zeta_{3}\right) F\left(z^{\prime}\right) \\
& \quad=\frac{F\left(\zeta_{1}\right)}{\left(\zeta_{1}-\zeta_{2}\right)\left(\zeta_{1}-\zeta_{3}\right)}+\frac{F\left(\zeta_{2}\right)}{\left(\zeta_{2}-\zeta_{3}\right)\left(\zeta_{2}-\zeta_{1}\right)}+\frac{F\left(\zeta_{3}\right)}{\left(\zeta_{3}-\zeta_{1}\right)\left(\zeta_{3}-\zeta_{2}\right)} \\
& D_{z}\left(\zeta_{1}, \zeta_{2}\right) D_{z^{\prime}}\left(z, \zeta_{3}\right) D_{z^{\prime \prime}}\left(z^{\prime}, \zeta_{4}\right) F\left(z^{\prime \prime}\right)=D_{z}\left(\zeta_{1}, \zeta_{2}\right) D_{z^{\prime}}\left(\zeta_{3}, \zeta_{4}\right) D_{z^{\prime \prime}}\left(z, z^{\prime}\right) F\left(z^{\prime \prime}\right) \\
& \quad=\frac{F\left(\zeta_{1}\right)}{\left(\zeta_{1}-\zeta_{2}\right)\left(\zeta_{1}-\zeta_{3}\right)\left(\zeta_{1}-\zeta_{4}\right)}+\frac{F\left(\zeta_{2}\right)}{\left(\zeta_{2}-\zeta_{3}\right)\left(\zeta_{2}-\zeta_{4}\right)\left(\zeta_{2}-\zeta_{1}\right)} \\
& \quad+\frac{F\left(\zeta_{3}\right)}{\left(\zeta_{3}-\zeta_{4}\right)\left(\zeta_{3}-\zeta_{1}\right)\left(\zeta_{3}-\zeta_{2}\right)}+\frac{F\left(\zeta_{4}\right)}{\left(\zeta_{4}-\zeta_{1}\right)\left(\zeta_{4}-\zeta_{2}\right)\left(\zeta_{4}-\zeta_{3}\right)},
\end{aligned}
$$

which are symmetric under interchange among $\zeta_{1}, \zeta_{2}, \zeta_{3}$ or $\zeta_{1}, \zeta_{2}, \zeta_{3}, \zeta_{4}$, respectively. Physically, these properties imply that the order of merging of three or four domains is irrelevant.

The commutativity we have just proven on the basis of the rules $(5.1) \sim(5.3)$ means that the interaction terms in the Hamiltonian in terms of the transformed fields $\left(\frac{\delta}{\delta \hat{J}}\right)_{I}$ or $\left(\frac{\delta}{\delta \hat{K}}\right)_{I}$ take the same form as those in the bare Hamiltonian in terms of $\left(\frac{\delta}{\delta J}\right)_{I}$ or $\left(\frac{\delta}{\delta K}\right)_{I}$, with a proviso that some of the terms present before the scaling limit can vanish after the scaling limit, as occurring in the $Y_{\alpha}$-derivative terms of our Hamiltonian. Since our proof is based on a general structure of the mixing matrix, being the exponential of a derivation operator $\mathcal{R}$, we strongly suspect that the commutativity is a universal property of the matrix models and their string field theories. Furthermore, we might think a transformation such as (4.1) as a special case of more general symmetry transformations of the string fields: If a linear transformation of string fields obeys the general rules (5.1) (5.3), the interaction terms of

5 The notations for the original model are used in these figures, but the argument is also valid for the dual one, after changing the variables $\zeta_{1}, \sigma, \zeta_{2}$ into $\xi_{1}, \xi_{2}, \xi_{3}$. 
the string field theory are invariant under the transformation, leaving aside the question of generating kinetic terms in attempting a background independent formulation of the string field theory as discussed in [6].

\begin{tabular}{|c|l|l|r|}
\hline Process & Original model & Dual model & Necessary Identities \\
\hline \hline \multirow{3}{*}{ Splitting } & $\mathrm{I}=\mathrm{n}(\mathrm{n} \geq 2)$ & $\mathrm{I}=\mathrm{n}(\mathrm{n} \geq 3)$ & $(5.9)$ \\
\cline { 2 - 4 } & $\mathrm{I}=\mathrm{n}(\mathrm{n} \geq 3)$ & $\mathrm{I}=\mathrm{n}(\mathrm{n} \geq 4)$ & $(5.10)$ \\
\hline \multirow{5}{*}{ Merging } & $(\mathrm{I}, \mathrm{J})=(\mathrm{n}, \mathrm{A}),(\mathrm{n}, \mathrm{B})(\mathrm{n} \geq 2)$ & $(\mathrm{I}, \mathrm{J})=(\mathrm{n}, \mathrm{X})(\mathrm{n} \geq 3)$ & $(5.11)$ \\
\cline { 2 - 4 } & & $(\mathrm{I}, \mathrm{J})=(2,1)$ & $(5.12)$ \\
\cline { 2 - 4 } & & $(\mathrm{I}, \mathrm{J})=(\mathrm{n}, 1)(\mathrm{n} \geq 3)$ & $(5.12)$ \\
\cline { 2 - 4 } & $(\mathrm{I}, \mathrm{J})=(1,1)$ & $(\mathrm{I}, \mathrm{J})=(2,2)$ & $(5.12),(5.13)$ \\
\cline { 2 - 4 } & $(\mathrm{I}, \mathrm{J})=(\mathrm{n}, 1)(\mathrm{n} \geq 2)$ & $(\mathrm{I}, \mathrm{J})=(\mathrm{n}, 2)(\mathrm{n} \geq 3)$ & $(5.13)$ \\
\cline { 2 - 4 } & $(\mathrm{I}, \mathrm{J})=(\mathrm{n}, \mathrm{m})(\mathrm{n}, \mathrm{m} \geq 2)$ & $(\mathrm{I}, \mathrm{J})=(\mathrm{n}, \mathrm{m})(\mathrm{n}, \mathrm{m} \geq 3)$ & \\
\hline
\end{tabular}

Table: Appearance of non-trivial identities.

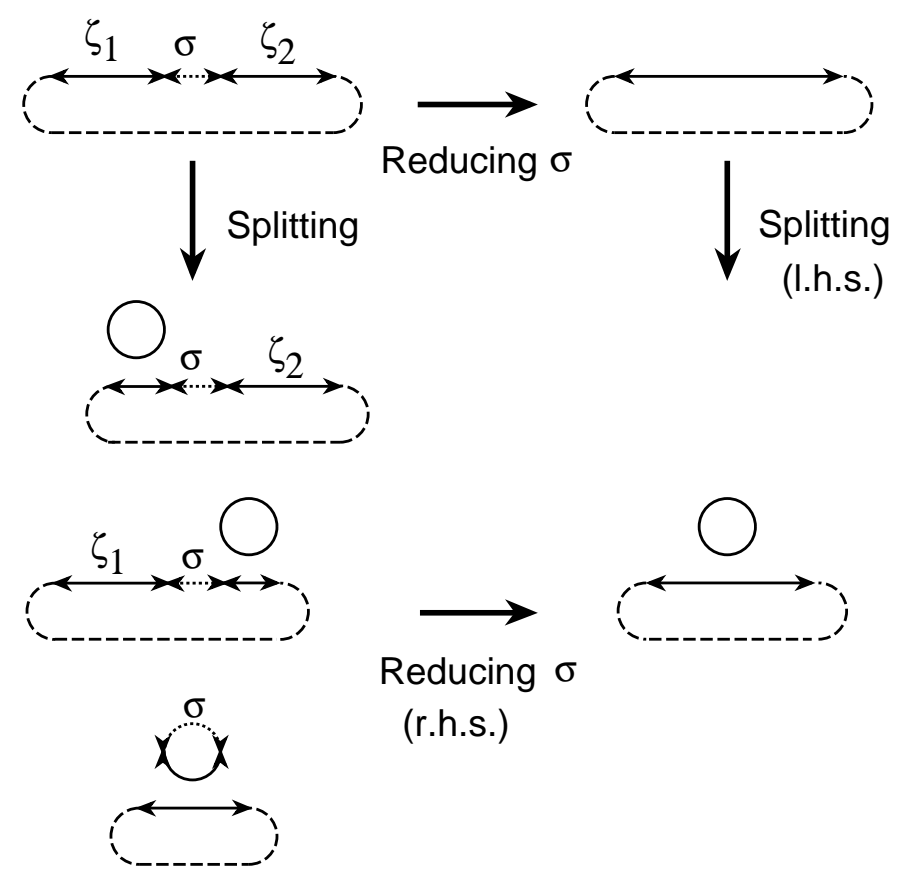

Figure 1: The pictorial explanation of the identity (5.9). This is required for the following cases. Suppose there are three successive domains with $\zeta_{1}, \sigma, \zeta_{2}$. The identity shows that the reduction of the domain with $\sigma$ commutes with the particular splitting processes which occur in the $\zeta_{1}$ domain or in the $\zeta_{2}$ domain. 

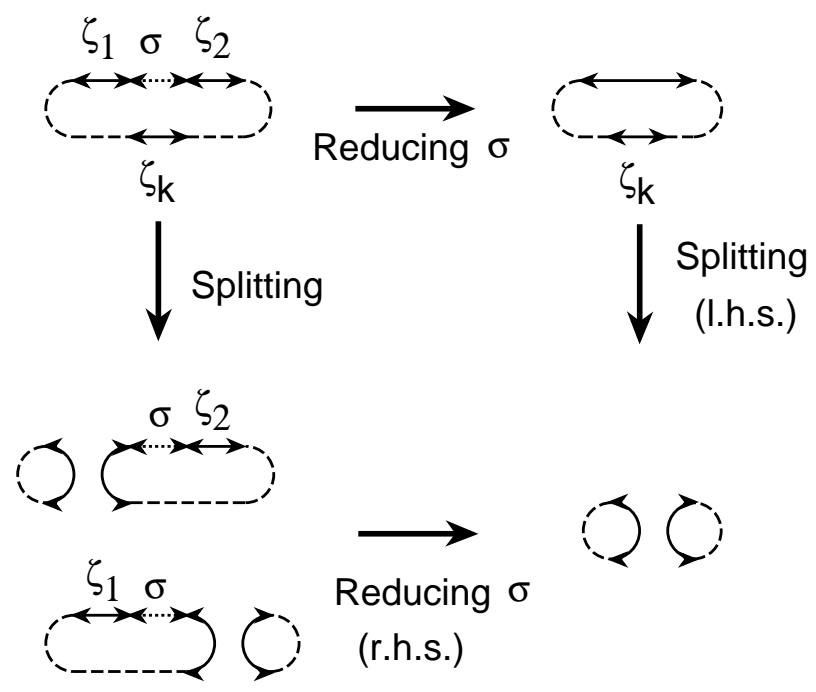

Figure 2: The pictorial explanation of the identity (5.10). Consider the three successive domains with $\zeta_{1}, \sigma, \zeta_{2}$ as in Figure 1. The identity shows that the reduction of the domain with $\sigma$ commutes with a splitting process between the domain with $\zeta_{1}$ and another domain with $\zeta_{k}$ and one between the domain with $\zeta_{2}$ and the domain with $\zeta_{k}$.

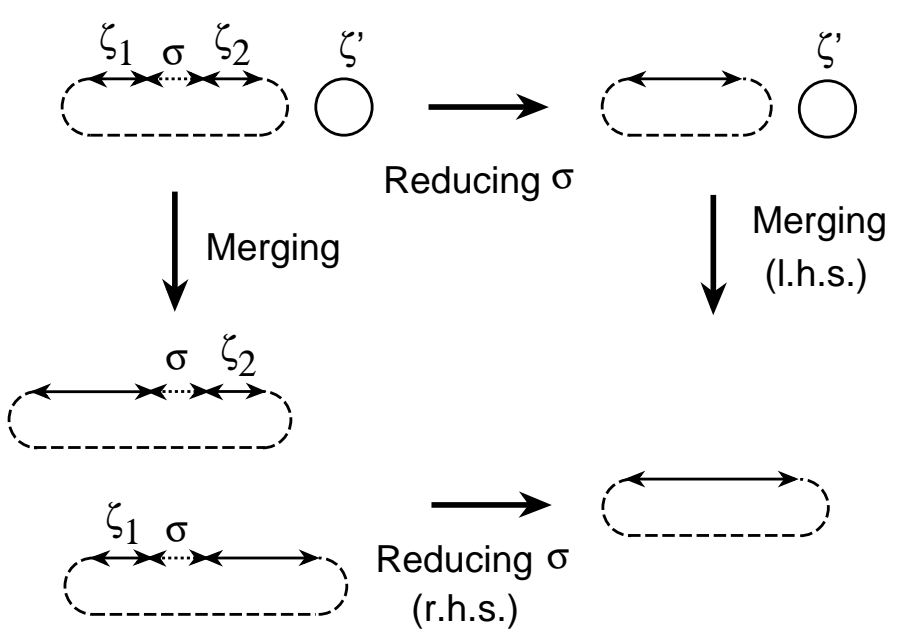

Figure 3: The pictorial explanation of the identity (5.11). This identity is required when one of two strings consists of only one domain with $\zeta^{\prime}$. (By "only one domain", we mean $\Psi_{X}$ (not $\left.\Psi_{1}\right)$ for the dual model.) Consider the three successive domains with $\zeta_{1}, \sigma, \zeta_{2}$ in the other string. Merging processes that the string with one domain merges into the $\zeta_{1}$ domain or the $\zeta_{2}$ domain commute with the reduction of the $\sigma$ domain. 


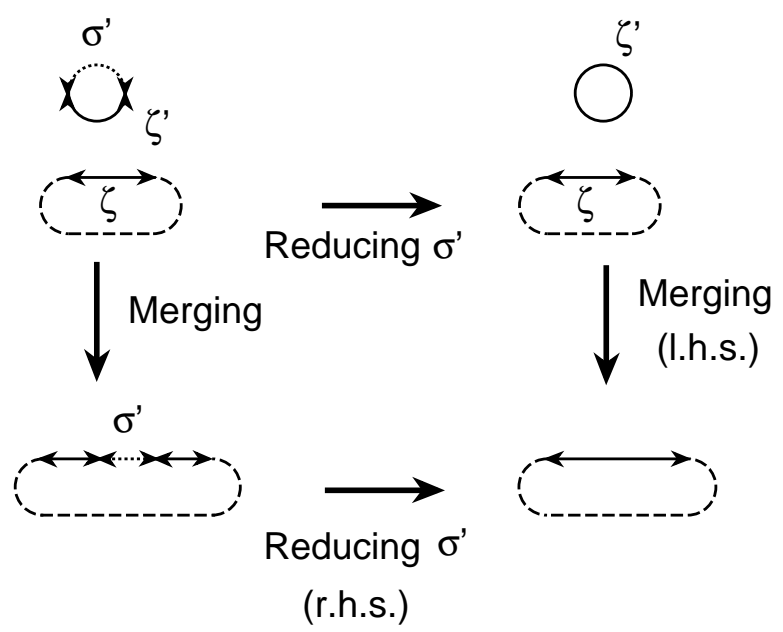

Figure 4: The pictorial explanation of the identity (5.12). When one of two strings consists of two domains with $\zeta^{\prime}$ and $\sigma^{\prime}$ and we consider the reduction of the $\sigma^{\prime}$ domain, we need this identity. We consider a merging process involving the $\zeta^{\prime}$ domain and a $\zeta$ domain in the other string. The identity shows that it commutes with the reduction of the domain with $\sigma^{\prime}$.

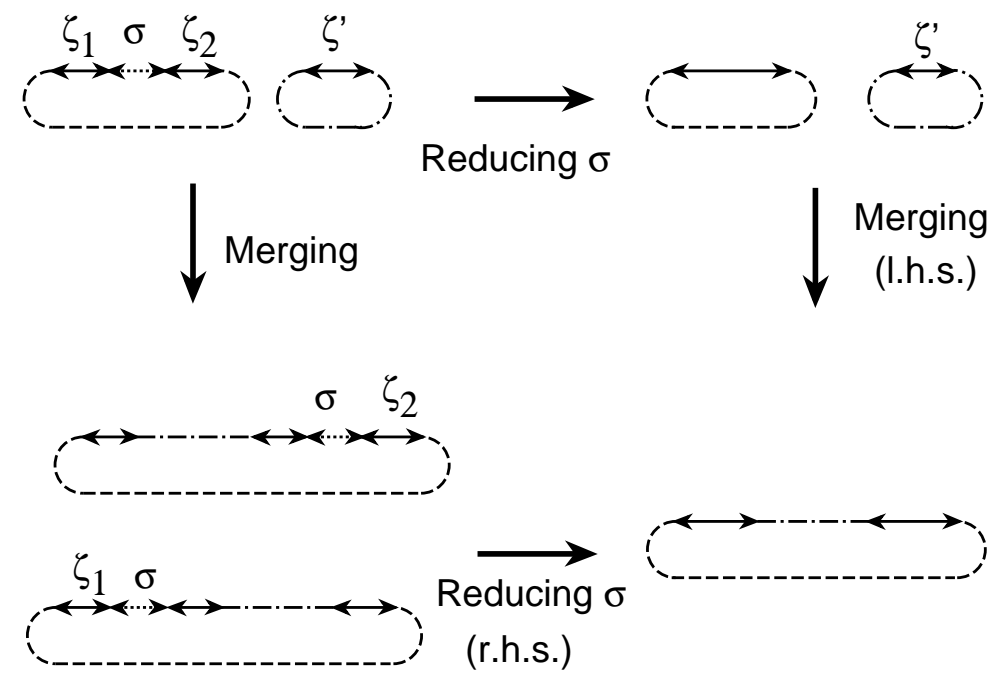

Figure 5: The pictorial explanation of the identity (5.13). We focus on the three successive domains with $\zeta_{1}, \sigma, \zeta_{2}$ in a string. We consider a merging process between the $\zeta_{1}$ domain and a $\zeta^{\prime}$ domain in the other string and one between the $\zeta_{2}$ domain and the $\zeta^{\prime}$ domain. This identity represents the commutativity of those merging processes with the reduction of the $\sigma$ domain. 


\section{DISCUSSION OF DUALITY}

In section IV, we have derived the string field Hamiltonian in the dual formalism. The result shows that the T-duality symmetry would be broken if the topological excitation of the $Y$-loops at the quantum level could not be neglected. Here, in order to check this, we shall calculate explicitly the disk amplitudes with one handle in both the original and dual theories. We will find that the topological $Y$-loops indeed contribute to the amplitude in the continuum theory. Also, a further correspondence between the original and dual Hamiltonians will be discussed.

\section{A. Amplitudes for cylinder and disk with one handle in the original theory}

We first derive the amplitude for disk with one handle in the original theory by taking the continuum limit of the Schwinger-Dyson equation. To do this, it is necessary to know the form of the cylinder amplitude $\left\langle\Phi_{A}(\zeta) \Phi_{A}\left(\zeta^{\prime}\right)\right\rangle_{0}$. The Schwinger-Dyson equation determining this is

$$
\begin{aligned}
0= & {\left[3\left\langle\Phi_{A}(\zeta)\right\rangle_{0}^{2}+2\left(-2 \zeta+2 g \zeta^{2}+\frac{c}{g}\right)\left\langle\Phi_{A}(\zeta)\right\rangle_{0}-g\left\langle\frac{1}{N} \operatorname{tr} A\right\rangle_{0}\right.} \\
& +1-g \zeta+\left(\zeta-g \zeta^{2}\right)\left(\zeta-g \zeta^{2}-\frac{c}{g}\right)+\frac{c^{3}}{g} \zeta\left\langle\Phi_{A}\left(\zeta^{\prime}\right) \Phi_{A}(\zeta)\right\rangle_{0} \\
& +\left[-g\left\langle\Phi_{A}(\zeta)\right\rangle_{0}+g\left(\zeta-g \zeta^{2}-\frac{c}{g}\right)-1+g \zeta\right]\left\langle\Phi_{A}\left(\zeta^{\prime}\right) \frac{1}{N} \operatorname{tr} A\right\rangle_{0} \\
& +g(2-g \zeta)\left\langle\Phi_{A}\left(\zeta^{\prime}\right) \frac{1}{N} \operatorname{tr} A^{2}\right\rangle_{0}-g^{2}\left\langle\Phi_{A}\left(\zeta^{\prime}\right) \frac{1}{N} \operatorname{tr} A^{3}\right\rangle_{0} \\
& +\frac{1}{N^{2}} \partial_{\zeta^{\prime}} D_{z}\left(\zeta, \zeta^{\prime}\right)\left[\left\langle\Phi_{A}(z)\right\rangle_{0}^{2}+\left\langle\Phi_{A}(\zeta)\right\rangle_{0}\left\langle\Phi_{A}(z)\right\rangle_{0}\right. \\
& \left.+\left(-2 z+2 g z^{2}+\frac{c}{g}\right)\left\langle\Phi_{A}(z)\right\rangle_{0}\right]
\end{aligned}
$$

where $\Phi_{A}(\zeta)=\frac{1}{N} \operatorname{tr} \frac{1}{\zeta-A}$, and the symbol $\langle\cdots\rangle_{h}$ represents a connected amplitude with $h$ handles. This is derived using a similar method as in the closed cubic equation for the disk amplitude $W(\zeta)$ [6]. Eq. (6.1) can be solved by using the following two facts. One is that the factor in front of $\left\langle\Phi_{A}\left(\zeta^{\prime}\right) \Phi_{A}(\zeta)\right\rangle_{0}$ becomes

$$
a^{8 / 3} \frac{3 c s^{8 / 3}}{40 \cdot 2^{2 / 3}}\left(w(y)^{2}-T^{4 / 3}\right)+O\left(a^{3}\right)
$$

in the continuum limit, where for the continuum limit in the original theory, we use the notation in Appendix C of Ref. [6]. For instance,

$$
\begin{gathered}
g=g_{*}\left(1-a^{2} \frac{s^{2}}{20} T\right), \quad \zeta=\frac{1+3 c}{2 g_{*}}(1+a y), \quad s=2+\sqrt{7}, \\
\left\langle\hat{\Phi}_{A}(\zeta)\right\rangle_{0}=a^{4 / 3} \frac{c^{1 / 2} s^{4 / 3}}{\sqrt{10} \cdot 2^{4 / 3}} \hat{w}(y),
\end{gathered}
$$


and $w(y)$ is the leading term of $\hat{w}(y)$ :

$$
w(y)=\left(y+\sqrt{y^{2}-T}\right)^{4 / 3}+\left(y-\sqrt{y^{2}-T}\right)^{4 / 3} .
$$

The other is that the leading term of $(6.2)$ vanishes at $y=0, \pm \sqrt{\frac{T}{2}} \cdot 6$ Using the scaling of the cylinder $\left\langle\Phi_{A}\left(\zeta^{\prime}\right) \Phi_{A}(\zeta)\right\rangle_{0}=O\left(\frac{1}{N^{2}} a^{-2}\right)$ and the above properties, we can easily see

$$
\left\langle\Phi_{A}(\zeta) \frac{1}{N} \operatorname{tr} A^{n}\right\rangle_{0}=O\left(\frac{1}{N^{2}} a^{-2 / 3}\right)
$$

for $n=1,2,3$. Then, note that Eq. (6.1) can be written as

$$
\begin{aligned}
0= & a^{8 / 3} \frac{3 c s^{8 / 3}}{40 \cdot 2^{2 / 3}}\left(w(y)^{2}-T^{4 / 3}\right)\left\langle\Phi_{A}\left(\zeta^{\prime}\right) \Phi_{A}(\zeta)\right\rangle_{0} \\
& -a^{4 / 3} \frac{c^{2} s^{4 / 3}}{2^{4 / 3}} w(y)\left\langle\Phi_{A}\left(\zeta^{\prime}\right) \frac{1}{N} \operatorname{tr} A\right\rangle_{0}+a c^{2} s y g_{2}\left(y^{\prime}\right)+c^{2} g_{3}\left(y^{\prime}\right) \\
& +\frac{1}{N^{2}} a^{2 / 3} \frac{c^{2} s^{2 / 3}}{4 \cdot 2^{2 / 3}} \partial_{y^{\prime}}\left[\frac{w(y)-w\left(y^{\prime}\right)}{y-y^{\prime}}\left(2 w(y)+w\left(y^{\prime}\right)\right)\right]+O\left(\frac{1}{N^{2}} a\right),
\end{aligned}
$$

where

$$
\begin{aligned}
g_{2}\left(y^{\prime}\right)= & 2 \sqrt{7}\left\langle\Phi_{A}\left(\zeta^{\prime}\right) \frac{1}{N} \operatorname{tr} A\right\rangle_{0}-\sqrt{10 c}\left\langle\Phi_{A}\left(\zeta^{\prime}\right) \frac{1}{N} \operatorname{tr} A^{2}\right\rangle_{0} \\
g_{3}\left(y^{\prime}\right)= & -4\left\langle\Phi_{A}\left(\zeta^{\prime}\right) \frac{1}{N} \operatorname{tr} A\right\rangle_{0}+\sqrt{70 c}\left\langle\Phi_{A}\left(\zeta^{\prime}\right) \frac{1}{N} \operatorname{tr} A^{2}\right\rangle_{0}-\frac{10}{3} c\left\langle\Phi_{A}\left(\zeta^{\prime}\right) \frac{1}{N} \operatorname{tr} A^{3}\right\rangle_{0} \\
& -\frac{1}{N^{2}} \frac{2}{3}+a^{1 / 3} \frac{1}{N^{2}} \frac{s^{1 / 3}}{3 \cdot 2^{1 / 3}} \partial_{y} \hat{w}(y) .
\end{aligned}
$$

From the consistency of Eq. (6.4), the nontrivial orders of $g_{2}$ and $g_{3}$ must be at most

$$
g_{2}(y)=O\left(\frac{1}{N^{2}} a^{-1 / 3}\right), \quad g_{3}(y)=O\left(\frac{1}{N^{2}} a^{2 / 3}\right) .
$$

Now, using the second of the above two facts, solving the equation (6.4) to the leading order is an easy task. We arrive at

$$
\begin{aligned}
\left\langle\Phi_{A}(\zeta) \frac{1}{N} \operatorname{tr} A\right\rangle_{0}= & \frac{1}{N^{2}} a^{-2 / 3} 2^{-4 / 3} s^{-2 / 3} \partial_{y}\left[y^{-1} w(y)\right. \\
& \left.+\frac{T^{1 / 3}}{4\left(y^{2}-T / 2\right) y}\left(w(y)^{2}+T^{2 / 3} w(y)-2 T^{4 / 3}\right)\right]+O\left(\frac{1}{N^{2}} a^{-1 / 3}\right),
\end{aligned}
$$

${ }^{6}$ The Riemann surface of the disk $w(y)$ consists of a 3 -sheeted plane. The first sheet has one cut in the region $y<-\sqrt{T}$ on the real axis, the second one has the two cuts $y<-\sqrt{T}$ and $y>\sqrt{T}$, and the third has the one cut $y>\sqrt{T}$. In this statement, it should be considered that $y=0$ and $\sqrt{\frac{T}{2}}$ are points on the first sheet but $y=-\sqrt{\frac{T}{2}}$ on the second sheet. Here, we derive the cylinder amplitude by assuming that it is regular at these three points. 


$$
\begin{aligned}
g_{2}(y)=- & \frac{1}{N^{2}} a^{-1 / 3} 2^{-8 / 3} s^{-1 / 3} \partial_{y}\left[\frac{1}{y^{2}-T / 2}\left(w(y)^{2}+T^{2 / 3} w(y)-2 T^{4 / 3}\right)\right] \\
& +O\left(\frac{1}{N^{2}} a^{0}\right), \\
g_{3}(y)= & -\frac{1}{N^{2}} a^{2 / 3} \frac{s^{2 / 3}}{12 \cdot 2^{2 / 3}} \partial_{y}\left[y^{-1}\left(w(y)^{2}-2 T^{4 / 3}\right)\right. \\
& \left.+\frac{T}{4\left(y^{2}-T / 2\right) y}\left(w(y)^{2}+T^{2 / 3} w(y)-2 T^{4 / 3}\right)\right]+O\left(\frac{1}{N^{2}} a\right), \\
\left\langle\Phi_{A}(\zeta) \Phi_{A}\left(\zeta^{\prime}\right)\right\rangle_{0}= & \frac{1}{N^{2}} a^{-2} \frac{10 c}{s^{2}} w\left(y, y^{\prime}\right)+O\left(\frac{1}{N^{2}} a^{-5 / 3}\right), \\
w\left(y, y^{\prime}\right)= & \frac{4}{9} \frac{1}{f\left(y, y^{\prime}\right) g\left(y, y^{\prime}\right)} \frac{z_{+}(y)^{1 / 3}}{z_{+}(y)^{2 / 3}+z_{-}(y)^{2 / 3}+T^{1 / 3}} \\
& \times \frac{z_{+}\left(y^{\prime}\right)^{1 / 3}}{z_{+}\left(y^{\prime}\right)^{2 / 3}+z_{-}\left(y^{\prime}\right)^{2 / 3}+T^{1 / 3}}\left[1+\frac{3 T^{1 / 3}}{f\left(y, y^{\prime}\right)}+3 \frac{z_{+}(y) z_{+}\left(y^{\prime}\right)}{g\left(y, y^{\prime}\right)}\right],
\end{aligned}
$$

where

$$
\begin{aligned}
z_{ \pm}(y) & =y \pm \sqrt{y^{2}-T} \\
f\left(y, y^{\prime}\right) & =z_{+}(y)^{1 / 3} z_{+}\left(y^{\prime}\right)^{1 / 3}+z_{-}(y)^{1 / 3} z_{-}\left(y^{\prime}\right)^{1 / 3}+T^{1 / 3} \\
g\left(y, y^{\prime}\right) & =z_{+}(y)^{2 / 3}+z_{+}\left(y^{\prime}\right)^{2 / 3}+z_{+}(y)^{1 / 3} z_{+}\left(y^{\prime}\right)^{1 / 3} .
\end{aligned}
$$

Next, let us proceed to the amplitude for disk with one handle. The Schwinger-Dyson equation we consider is

$$
\begin{aligned}
0= & {\left[3\left\langle\Phi_{A}(\zeta)\right\rangle_{0}^{2}+2\left(-2 \zeta+2 g \zeta^{2}+\frac{c}{g}\right)\left\langle\Phi_{A}(\zeta)\right\rangle_{0}-g\left\langle\frac{1}{N} \operatorname{tr} A\right\rangle_{0}\right.} \\
& +1-g \zeta+\left(\zeta-g \zeta^{2}\right)\left(\zeta-g \zeta^{2}-\frac{c}{g}\right)+\frac{c^{3}}{g} \zeta\left\langle\Phi_{A}(\zeta)\right\rangle_{1} \\
& +\left[3\left\langle\Phi_{A}(\zeta)\right\rangle_{0}+\frac{c}{g}-2 \zeta+2 g \zeta^{2}\right]\left\langle\Phi_{A}(\zeta)^{2}\right\rangle_{0} \\
& -g\left\langle\Phi_{A}(\zeta) \frac{1}{N} \operatorname{tr} A\right\rangle_{0}-g\left\langle\Phi_{A}(\zeta)\right\rangle_{0}\left\langle\frac{1}{N} \operatorname{tr} A\right\rangle_{1}+\frac{1}{N^{2}} \frac{1}{2} \partial_{\zeta}^{2}\left\langle\Phi_{A}(\zeta)\right\rangle_{0} \\
& +\left[g\left(\zeta-g \zeta^{2}-\frac{c}{g}\right)-c(1-g \zeta)+1-c\right]\left\langle\frac{1}{N} \operatorname{tr} A\right\rangle_{1}-g^{2}\left\langle\frac{1}{N} \operatorname{tr} A^{3}\right\rangle_{1} .
\end{aligned}
$$

Note that the factor in front of $\left\langle\Phi_{A}(\zeta)\right\rangle_{1}$ is the same as the first term in Eq. (6.1). This equation can then be solved by the same technique as before. The result is

$$
\begin{gathered}
\left\langle\frac{1}{N} \operatorname{tr} A\right\rangle_{1}=\frac{1}{N^{2}} a^{-2} \frac{\sqrt{10}}{24 c^{1 / 2} s^{2}} T^{-1}+O\left(\frac{1}{N^{2}} a^{-4 / 3}\right), \\
\left\langle\frac{1}{N} \operatorname{tr} A\right\rangle_{1}-\frac{c}{3}\left\langle\frac{1}{N} \operatorname{tr} A^{3}\right\rangle_{1}=-\frac{1}{N^{2}} a^{-2 / 3} \frac{\sqrt{10}}{216 \cdot 2^{7 / 3} c^{1 / 2} s^{2 / 3}} T^{-1 / 3}+O\left(\frac{1}{N^{2}} a^{0}\right),
\end{gathered}
$$




$$
\left\langle\Phi_{A}(\zeta)\right\rangle_{1}=\frac{1}{N^{2}} a^{-10 / 3} \frac{40 \sqrt{10 c}}{2^{2 / 3} s^{10 / 3}} w_{(1)}(y)+O\left(\frac{1}{N^{2}} a^{-3}\right),
$$

where

$$
\begin{aligned}
w_{(1)}(y)= & \frac{1}{216 T} \frac{1}{\left(z_{+}(y)^{2 / 3}+z_{-}(y)^{2 / 3}+T^{1 / 3}\right)^{5}}\left[12 y^{2}+77 T\right. \\
& \left.+15 T^{1 / 3}\left(z_{+}(y)^{4 / 3}+z_{-}(y)^{4 / 3}\right)+50 T^{2 / 3}\left(z_{+}(y)^{2 / 3}+z_{-}(y)^{2 / 3}\right)\right] .
\end{aligned}
$$

\section{B. Amplitudes for cylinder and disk with one handle in the dual theory}

On the other hand, in the dual theory, the Schwinger-Dyson equation for the cylinder is

$$
\begin{aligned}
0=[ & -3 \hat{g}\left\langle\Psi_{X}(\xi)\right\rangle_{0}^{2}+2\left(2 c(1+c)-\hat{g}^{2} \xi^{2}+(1-5 c) \hat{g} \xi\right)\left\langle\Psi_{X}(\xi)\right\rangle_{0} \\
& \left.+2 \hat{g}^{2}\left\langle\frac{1}{N} \operatorname{tr} X\right\rangle_{0}-\hat{g}(1-3 c)-2 c \xi(1+c-2 \hat{g} \xi)(1-c-\hat{g} \xi)\right] \\
& \times\left\langle\Psi_{X}\left(\xi^{\prime}\right) \Psi_{X}(\xi)\right\rangle_{0} \\
& +\hat{g}\left[2 \hat{g}\left\langle\Psi_{X}(\xi)\right\rangle_{0}-2 c(3-c)+4 c \hat{g} \xi\right]\left\langle\Psi_{X}\left(\xi^{\prime}\right) \frac{1}{N} \operatorname{tr} X\right\rangle_{0} \\
+ & 4 c \hat{g}^{2}\left\langle\Psi_{X}\left(\xi^{\prime}\right) \frac{1}{N} \operatorname{tr} X^{2}\right\rangle_{0}+\hat{g}^{2} \frac{1}{N^{2}} \partial_{\xi^{\prime}}\left\langle\frac{1}{N} \operatorname{tr}\left(\frac{1}{\xi^{\prime}-X} Y \frac{1}{\xi-X} Y\right)\right\rangle_{0} \\
& +\frac{1}{N^{2}} \partial_{\xi^{\prime}} D_{z}\left(\xi, \xi^{\prime}\right)\left[-\hat{g}\left\langle\Psi_{X}(z)\right\rangle_{0}^{2}-\hat{g}\left\langle\Psi_{X}(\xi)\right\rangle_{0}\left\langle\Psi_{X}(z)\right\rangle_{0}\right. \\
& \left.+\left(2 c(1+c)-\hat{g}^{2} z^{2}+(1-5 c) \hat{g} z\right)\left\langle\Psi_{X}(z)\right\rangle_{0}\right]
\end{aligned}
$$

which is also derived in a similar way as obtaining Eq. (B2). Note that the term

$$
\hat{g}^{2} \frac{1}{N^{2}} \partial_{\xi^{\prime}}\left\langle\frac{1}{N} \operatorname{tr}\left(\frac{1}{\xi^{\prime}-X} Y \frac{1}{\xi-X} Y\right)\right\rangle_{0}
$$

comes from the following Schwinger-Dyson equation

$$
0=\int d^{N^{2}} X d^{N^{2}} Y \sum_{\alpha} \frac{\partial}{\partial X_{\alpha}}\left[\operatorname{tr} \frac{1}{\xi^{\prime}-X} \operatorname{tr}\left(\frac{1}{\xi-X} Y t^{\alpha} Y\right) \mathrm{e}^{-S_{D}}\right]
$$

which reflects the fact that the dual theory does not distinguish overall spin directions. When both domains of $\xi$ and $\xi^{\prime}$ are of spin-up (spin-down), such a term does not appear in the original theory. As we will see, this survives in the scaling limit and leads to the difference between the cylinder amplitudes.

Since the scaling limit of the factor in front of $\left\langle\Psi_{X}\left(\xi^{\prime}\right) \Psi_{X}(\xi)\right\rangle_{0}$ becomes

$$
a^{8 / 3} \frac{3 c^{5 / 2} \hat{s}^{8 / 3}}{2^{4 / 3} \sqrt{5}}\left(\hat{T}^{4 / 3}-v(y)^{2}\right)+O\left(a^{3}\right)
$$

Eq. (6.15) can be solved by the same argument as before. We have the following result: 


$$
\begin{gathered}
\left\langle\Psi_{X}(\xi) \frac{1}{N} \operatorname{tr} X\right\rangle_{0}=\frac{1}{N^{2}} a^{-2 / 3} 2^{-2 / 3} \hat{s}^{-2 / 3} \partial_{y}\left[y^{-1} v(y)\right. \\
\left.+\frac{\hat{T}^{1 / 3}}{4\left(y^{2}-\hat{T} / 2\right) y}\left(v(y)^{2}+\hat{T}^{2 / 3} v(y)-2 \hat{T}^{4 / 3}\right)\right] \\
+O\left(\frac{1}{N^{2}} a^{-1 / 3}\right), \\
-108 c\left\langle\Psi_{X}(\xi) \frac{1}{N} \operatorname{tr} X\right\rangle_{0}+324 \sqrt{5 c}\left\langle\Psi_{X}(\xi) \frac{1}{N} \operatorname{tr} X^{2}\right\rangle_{0}+\frac{4}{N^{2}} \\
=\frac{1}{N^{2}} a^{2 / 3} \hat{s}^{2 / 3} 2^{-1 / 3} \partial_{y}\left[v(y)^{2}-2 \hat{T}^{4 / 3}+\frac{\hat{T}}{4\left(y^{2}-\hat{T} / 2\right)}\left(v(y)^{2}+\hat{T}^{2 / 3} v(y)-2 \hat{T}^{4 / 3}\right)\right] \\
+O\left(\frac{1}{N^{2}} a\right), \\
\left\langle\Psi_{X}\left(\xi^{\prime}\right) \Psi_{X}(\xi)\right\rangle_{0}=\frac{1}{N^{2}} a^{-2} \hat{s}^{-2} 5 c\left[2 w_{D}\left(y, y^{\prime}\right)+u_{D}\left(y, y^{\prime}\right)\right]+O\left(\frac{1}{N^{2}} a^{-5 / 3}\right),
\end{gathered}
$$

where $w_{D}\left(y, y^{\prime}\right)$ is the result of the replacement $T \rightarrow \hat{T}$ in $w\left(y, y^{\prime}\right)$, and $u_{D}\left(y, y^{\prime}\right)$ represents the difference of the functional forms between the cylinder amplitudes:

$$
\begin{aligned}
u_{D}\left(y, y^{\prime}\right)=- & \frac{4}{9} \frac{y}{v(y)+\hat{T}^{2 / 3}} \frac{y^{\prime}}{v\left(y^{\prime}\right)+\hat{T}^{2 / 3}} \frac{1}{\left(y^{2}-y^{2}\right)^{2}} \\
& \times\left[\hat{z}_{+}(y)^{8 / 3}+\hat{z}_{-}(y)^{8 / 3}+\hat{z}_{+}\left(y^{\prime}\right)^{8 / 3}+\hat{z}_{-}\left(y^{\prime}\right)^{8 / 3}\right. \\
& -6\left(\hat{z}_{+}(y)^{4 / 3}+\hat{z}_{-}(y)^{4 / 3}\right)\left(\hat{z}_{+}\left(y^{\prime}\right)^{4 / 3}+\hat{z}_{-}\left(y^{\prime}\right)^{4 / 3}\right) \\
& -5 \hat{T}^{2 / 3}\left(\hat{z}_{+}(y)^{4 / 3}+\hat{z}_{-}(y)^{4 / 3}+\hat{z}_{+}\left(y^{\prime}\right)^{4 / 3}+\hat{z}_{-}\left(y^{\prime}\right)^{4 / 3}\right) \\
& +6 \hat{T}^{2 / 3}\left(\hat{z}_{+}(y)^{2 / 3}+\hat{z}_{-}(y)^{2 / 3}\right)\left(\hat{z}_{+}\left(y^{\prime}\right)^{2 / 3}+\hat{z}_{-}\left(y^{\prime}\right)^{2 / 3}\right) \\
& +4\left(2 y^{2}-\hat{T}\right)\left(\hat{z}_{+}\left(y^{\prime}\right)^{2 / 3}+\hat{z}_{-}\left(y^{\prime}\right)^{2 / 3}\right) \\
& \left.+4\left(\hat{z}_{+}(y)^{2 / 3}+\hat{z}_{-}(y)^{2 / 3}\right)\left(2 y^{\prime 2}-\hat{T}\right)\right] \\
& +\frac{1}{\left(y+y^{\prime}\right)^{2}},
\end{aligned}
$$

where $\hat{z}_{ \pm}(y)$ is obtained by replacing $T$ to $\hat{T}$ in $z_{ \pm}(y)$. As will be mentioned later, the difference is interpreted as coming from the odd $Y$-loop excitations on a nontrivial homology cycle of the cylinder.

Also, for the disk with one handle, we can solve the Schwinger-Dyson equation:

$$
\begin{aligned}
0= & {\left[-3 \hat{g}\left\langle\Psi_{X}(\xi)\right\rangle_{0}^{2}+2\left(2 c(1+c)-\hat{g}^{2} \xi^{2}+(1-5 c) \hat{g} \xi\right)\left\langle\Psi_{X}(\xi)\right\rangle_{0}\right.} \\
& \left.+2 \hat{g}^{2}\left\langle\frac{1}{N} \operatorname{tr} X\right\rangle_{0}-\hat{g}(1-3 c)-2 c \xi(1+c-2 \hat{g} \xi)(1-c-\hat{g} \xi)\right]\left\langle\Psi_{X}(\xi)\right\rangle_{1} \\
& +\left[-3 \hat{g}\left\langle\Psi_{X}(\xi)\right\rangle_{0}+2 c(1+c)-\hat{g}^{2} \xi^{2}+(1-5 c) \hat{g} \xi\right]\left\langle\Psi_{X}(\xi)^{2}\right\rangle_{0} \\
& +2 \hat{g}^{2}\left\langle\Psi_{X}(\xi) \frac{1}{N} \operatorname{tr} X\right\rangle_{0}-\hat{g}^{2}\left\langle\left(\frac{1}{N} \operatorname{tr} \frac{1}{\xi-X} Y\right)^{2}\right\rangle_{0}-\hat{g} \frac{1}{N^{2}} \frac{1}{2} \partial_{\xi}^{2}\left\langle\Psi_{X}(\xi)\right\rangle_{0} \\
& +\left[2 \hat{g}^{2}\left\langle\Psi_{X}(\xi)\right\rangle_{0}+2 c \hat{g}(-3+c+2 \hat{g} \xi)\right]\left\langle\frac{1}{N} \operatorname{tr} X\right\rangle_{1}+4 c \hat{g}^{2}\left\langle\frac{1}{N} \operatorname{tr} X^{2}\right\rangle_{1} .
\end{aligned}
$$


Note the appearance of the term $-\hat{g}^{2}\left\langle\left(\frac{1}{N} \operatorname{tr} \frac{1}{\xi-X} Y\right)^{2}\right\rangle_{0}$, which does not allow the interpretation in terms of dual spins.

The solution is as follows:

$$
\begin{gathered}
\left\langle\frac{1}{N} \operatorname{tr} X\right\rangle_{1}=\frac{1}{N^{2}} a^{-2} \frac{\sqrt{5}}{24 c^{1 / 2} \hat{s}^{2}} \hat{T}^{-1}+O\left(\frac{1}{N^{2}} a^{-4 / 3}\right), \\
-108 \sqrt{5} c^{3 / 2}\left\langle\frac{1}{N} \operatorname{tr} X\right\rangle_{1}+20 c\left\langle\frac{1}{N} \operatorname{tr} X^{2}\right\rangle_{1} \\
=-\frac{1}{N^{2}} a^{-2 / 3} \hat{s}^{-2 / 3} \frac{25}{36 \cdot 2^{2 / 3}} \hat{T}^{-1 / 3}+O\left(\frac{1}{N^{2}} a^{0}\right), \\
\left\langle\Psi_{X}(\xi)\right\rangle_{1}=\frac{1}{N^{2}} a^{-10 / 3} \hat{s}^{-10 / 3} 5^{3 / 2} \cdot 2^{2 / 3} c^{1 / 2}\left[2 w_{(1) D}(y)+u_{(1)}(y)\right]+O\left(\frac{1}{N^{2}} a^{-3}\right),
\end{gathered}
$$

where $w_{(1) D}(y)$ is obtained by replacing $T$ to $\hat{T}$ in $w_{(1)}(y)$, and the difference from the original theory is

$$
u_{(1)}(y)=-\frac{1}{54 \hat{T}^{1 / 3}} \frac{5\left(\hat{z}_{+}(y)^{2 / 3}+\hat{z}_{-}(y)^{2 / 3}\right)+11 \hat{T}^{1 / 3}}{\left(\hat{z}_{+}(y)^{2 / 3}+\hat{z}_{-}(y)^{2 / 3}+\hat{T}^{1 / 3}\right)^{5}}
$$

\section{Comparison of the amplitudes}

We have obtained the amplitudes for cylinder and disk with one handle both in the original and dual theories. The result of the disk with one handle illustrates that the T-duality symmetry does not hold in the higher genus amplitudes as is expected from the discussion in section II. On the other hand, the difference between the cylinder amplitudes seems to be puzzling. This is resolved as follows. Since the dual theory can not discriminate overall dual spin directions, it is necessary to take account of the cylinder amplitude $\left\langle\Phi_{B}\left(\zeta^{\prime}\right) \Phi_{A}(\zeta)\right\rangle_{0}$, in comparing correlators with the original theory. The Schwinger-Dyson equation needed to obtain this is

$$
\begin{aligned}
0= & {\left[3\left\langle\Phi_{A}(\zeta)\right\rangle_{0}^{2}+2\left(-2 \zeta+2 g \zeta^{2}+\frac{c}{g}\right)\left\langle\Phi_{A}(\zeta)\right\rangle_{0}-g\left\langle\frac{1}{N} \operatorname{tr} A\right\rangle_{0}\right.} \\
& +1-g \zeta+\left(\zeta-g \zeta^{2}\right)\left(\zeta-g \zeta^{2}-\frac{c}{g}\right)+\frac{c^{3}}{g} \zeta\left\langle\Phi_{B}\left(\zeta^{\prime}\right) \Phi_{A}(\zeta)\right\rangle_{0} \\
& +\left[-g\left\langle\Phi_{A}(\zeta)\right\rangle_{0}+g\left(\zeta-g \zeta^{2}-\frac{c}{g}\right)-1+g \zeta\right]\left\langle\Phi_{B}\left(\zeta^{\prime}\right) \frac{1}{N} \operatorname{tr} A\right\rangle_{0} \\
& +g(2-g \zeta)\left\langle\Phi_{B}\left(\zeta^{\prime}\right) \frac{1}{N} \operatorname{tr} A^{2}\right\rangle_{0}-g^{2}\left\langle\Phi_{B}\left(\zeta^{\prime}\right) \frac{1}{N} \operatorname{tr} A^{3}\right\rangle_{0} \\
& -\frac{c^{2}}{g} \frac{1}{N^{2}} \partial_{\zeta^{\prime}}\left\langle\frac{1}{N} \operatorname{tr}\left(\frac{1}{\zeta-A} \frac{1}{\zeta^{\prime}-B}\right)\right\rangle_{0}
\end{aligned}
$$

The way to solve this is the same as before. We obtain 


$$
\begin{aligned}
& \left\langle\Phi_{B}(\zeta) \frac{1}{N} \operatorname{tr} A\right\rangle_{0}=\frac{1}{N^{2}} a^{-2 / 3} 2^{-4 / 3} s^{-2 / 3} \partial_{y}\left[y^{-1} w(y)\right. \\
& \left.+\frac{T^{1 / 3}}{4\left(y^{2}-T / 2\right) y}\left(w(y)^{2}+T^{2 / 3} w(y)-2 T^{4 / 3}\right)\right] \\
& +O\left(\frac{1}{N^{2}} a^{-1 / 3}\right), \\
& 2 \sqrt{7}\left\langle\Phi_{B}(\zeta) \frac{1}{N} \operatorname{tr} A\right\rangle_{0}-\sqrt{10 c}\left\langle\Phi_{B}(\zeta) \frac{1}{N} \operatorname{tr} A^{2}\right\rangle_{0} \\
& =-\frac{1}{N^{2}} a^{-1 / 3} 2^{-8 / 3} s^{-1 / 3} \partial_{y}\left[\frac{1}{y^{2}-T / 2}\left(w(y)^{2}+T^{2 / 3} w(y)-2 T^{4 / 3}\right)\right] \\
& +O\left(\frac{1}{N^{2}} a^{0}\right), \\
& -4\left\langle\Phi_{B}(\zeta) \frac{1}{N} \operatorname{tr} A\right\rangle_{0}+\sqrt{70 c}\left\langle\Phi_{B}(\zeta) \frac{1}{N} \operatorname{tr} A^{2}\right\rangle_{0}-\frac{10}{3} c\left\langle\Phi_{B}(\zeta) \frac{1}{N} \operatorname{tr} A^{3}\right\rangle_{0} \\
& -\frac{1}{N^{2}} \frac{1}{3}-a^{1 / 3} \frac{1}{N^{2}} \frac{s^{1 / 3}}{3 \cdot 2^{4 / 3}} \partial_{y} \hat{w}(y) \\
& =-\frac{1}{N^{2}} a^{2 / 3} \frac{s^{2 / 3}}{12 \cdot 2^{2 / 3}} \partial_{y}\left[y^{-1}\left(w(y)^{2}-2 T^{4 / 3}\right)+\frac{T}{4\left(y^{2}-T / 2\right) y}\left(w(y)^{2}+T^{2 / 3} w(y)-2 T^{4 / 3}\right)\right] \\
& +O\left(\frac{1}{N^{2}} a\right),
\end{aligned}
$$

and at the leading order the Schwinger-Dyson equation for $\left\langle\Phi_{B}\left(\zeta^{\prime}\right) \Phi_{A}(\zeta)\right\rangle_{0}+\left\langle\Phi_{A}\left(\zeta^{\prime}\right) \Phi_{A}(\zeta)\right\rangle_{0}$ becomes the same form as that for $\left\langle\Psi_{X}\left(\zeta^{\prime}\right) \Psi_{X}(\zeta)\right\rangle_{0}$. Thus, we have

$$
\left\langle\Phi_{B}\left(\zeta^{\prime}\right) \Phi_{A}(\zeta)\right\rangle_{0}+\left\langle\Phi_{A}\left(\zeta^{\prime}\right) \Phi_{A}(\zeta)\right\rangle_{0}=\frac{1}{N^{2}} a^{-2} \frac{10 c}{s^{2}}\left[2 w\left(y, y^{\prime}\right)+u\left(y, y^{\prime}\right)\right]+O\left(\frac{1}{N^{2}} a^{-5 / 3}\right),
$$

where $u\left(y, y^{\prime}\right)$ is obtained by replacing $\hat{T}$ to $T$ in $u_{D}\left(y, y^{\prime}\right)$.

It can now be seen that the mixed cylinder amplitude

$$
\left\langle\left(\Phi_{A}(\zeta)+\Phi_{B}(\zeta)\right)\left(\Phi_{A}\left(\zeta^{\prime}\right)+\Phi_{B}\left(\zeta^{\prime}\right)\right)\right\rangle_{0}
$$

of the original theory has the same functional form as $\left\langle\Psi_{X}(\xi) \Psi_{X}\left(\xi^{\prime}\right)\right\rangle_{0}$ of the dual theory, which means the duality symmetry in the cylinder level. On the cylinder in the dual theory, $Y$-loops along a nontrivial homology cycle can exit. A natural interpretation is that the amplitude with the even number $Y$-loops corresponds to $\left\langle\Phi_{A} \Phi_{A}\right\rangle_{0}+\left\langle\Phi_{B} \Phi_{B}\right\rangle_{0}$ in the original theory, and that the amplitude with the odd number ones to the cross term $\left\langle\Phi_{A} \Phi_{B}\right\rangle_{0}+$ $\left\langle\Phi_{B} \Phi_{A}\right\rangle_{0}$. This is consistent with the picture of the dual spins.

We can thus conclude that, to the leading order of the universal parts of the disk and cylinder amplitudes, the following identification between the original and dual theories holds:

$$
\begin{gathered}
T \Leftrightarrow \hat{T}, \\
\frac{s}{\sqrt{2}} \Leftrightarrow \hat{s},
\end{gathered}
$$




$$
\begin{gathered}
\frac{1}{\sqrt{2}}\left(\Phi_{A}(\zeta)+\Phi_{B}(\zeta)\right) \Leftrightarrow \Psi_{X}(\xi), \\
\frac{1}{\sqrt{2}} \operatorname{tr}(A+B) \Leftrightarrow \operatorname{tr} X .
\end{gathered}
$$

This can be checked for all the disk and cylinder amplitudes we have obtained.

\section{Comparison of the string field Hamiltonians}

Until now, after a long analysis of the Schwinger-Dyson equations, we have shown that the T-duality symmetry holds in some disk and cylinder amplitudes without handles, and that it is violated in the higher genus amplitude. Here, we make sure that in general the T-duality is a symmetry in the planar approximation, by comparing the forms of the string field Hamiltonians.

We start with the Hamiltonian of the original theory, where the numerical constant $\mathrm{cs}^{-1}$ in the kinetic term is dropped, since it can be absorbed into the finite renormalization of the fictitious time by rescaling as

$$
\tilde{J}_{I} \rightarrow \frac{1}{c s^{-1}} \tilde{J}_{I}, \quad \frac{\delta}{\delta \tilde{J}_{I}} \rightarrow c s^{-1} \frac{\delta}{\delta \tilde{J}_{I}}, \quad g_{\mathrm{st}} \rightarrow c s^{-1} g_{\mathrm{st}} .
$$

We introduce the string fields which have a definite parity with respect to the reversal $(A \leftrightarrow B)$ of spin directions:

$$
\begin{aligned}
& \tilde{J}_{X}^{( \pm)}(y)=\tilde{J}_{A}(y) \pm \tilde{J}_{B}(y) \\
& \tilde{J}_{2 n}^{( \pm)}\left(y_{1}, y_{2}, \cdots, y_{2 n}\right)=\frac{1}{2}\left(\tilde{J}_{n}\left(y_{1}, y_{2}, \cdots, y_{2 n}\right) \pm \tilde{J}_{n}\left(y_{2}, \cdots, y_{2 n}, y_{1}\right)\right), \\
& \frac{\delta}{\delta \tilde{J}_{X}^{( \pm)}(y)}=\frac{1}{2}\left(\frac{\delta}{\delta \tilde{J}_{A}(y)} \pm \frac{\delta}{\delta \tilde{J}_{B}(y)}\right) \\
& \frac{\delta}{\delta \tilde{J}_{2 n}^{( \pm)}\left(y_{1}, y_{2}, \cdots, y_{2 n}\right)}=\frac{1}{2}\left[\frac{\delta}{\delta \tilde{J}_{n}\left(y_{1}, y_{2}, \cdots, y_{2 n}\right)} \pm \frac{\delta}{\delta \tilde{J}_{n}\left(y_{2}, \cdots, y_{2 n}, y_{1}\right)}\right] \\
&(n=1,2,3, \cdots) .
\end{aligned}
$$

Recalling that the variable $\tilde{J}_{n}\left(y_{1}, \cdots, y_{2 n}\right)$ in the original theory have the cyclic symmetry with respect to the permutations of $n$-pairs $\left(y_{1}, y_{2}\right),\left(y_{3}, y_{4}\right), \cdots,\left(y_{2 n-1}, y_{2 n}\right)$, we see that the new variables $\tilde{J}^{( \pm)}$'s and the derivatives with respect to them have a definite symmetric or anti-symmetric property under the cyclic permutations of the $2 n$ variables $y_{1}, y_{2}, \cdots, y_{2 n}$. Note that the symmetric property of $\tilde{J}^{(+)}$'s is identical with that of the string fields in the dual theory.

Then the functional derivatives of the new variables become

$$
\begin{aligned}
\frac{\delta \tilde{J}_{X}^{(P)}(y)}{\delta \tilde{J}_{X}^{(Q)}\left(y^{\prime}\right)} & =2 \pi i \delta_{P, Q} \delta\left(y-y^{\prime}\right), \\
\frac{\delta \tilde{J}_{2 n}^{(+)}\left(y_{1}, \cdots, y_{2 n}\right)}{\delta \tilde{J}_{2 n}^{(+)}\left(y_{1}^{\prime}, \cdots, y_{2 n}^{\prime}\right)} & =\frac{1}{2 n}(2 \pi i)^{2 n} \sum_{c} \delta\left(y_{1}-y_{c(1)}^{\prime}\right) \ldots \delta\left(y_{2 n}-y_{c(2 n)}^{\prime}\right), \\
\frac{\delta \tilde{J}_{2 n}^{(-)}\left(y_{1}, \cdots, y_{2 n}\right)}{\delta \tilde{J}_{2 n}^{(-)}\left(y_{1}^{\prime}, \cdots, y_{2 n}^{\prime}\right)} & =\frac{1}{2 n}(2 \pi i)^{2 n} \sum_{c}(-1)^{|c|} \delta\left(y_{1}-y_{c(1)}^{\prime}\right) \ldots \delta\left(y_{2 n}-y_{c(2 n)}^{\prime}\right),
\end{aligned}
$$


and the others vanish, where $P$ and $Q$ take + or - , and $c$ represents the cyclic permutations of $1, \cdots, 2 n$. The notation $(-1)^{|c|}$ denotes its sign, namely, $(+1)$ for even cyclic permutations or $(-1)$ for odd cyclic permutations.

By using these variables, the Hamiltonian is written in the following form:

$$
\begin{aligned}
\mathcal{H}= & \mathcal{H}^{(+)}+\mathcal{H}^{(-)} \\
\mathcal{H}^{(+)}= & -\tilde{J}^{(+)} \cdot\left(F \frac{\delta}{\delta \tilde{J}^{(+)}}\right)-\tilde{J}^{(+)} \cdot\left(\frac{\delta}{\delta \tilde{J}^{(+)}} \vee \frac{\delta}{\delta \tilde{J}^{(+)}}\right)-\frac{1}{2} g_{\mathrm{st}}^{2} \tilde{J}^{(+)} \cdot\left(\tilde{J}^{(+)} \cdot\left(\wedge \frac{\delta}{\delta \tilde{J}^{(+)}}\right)\right), \\
\mathcal{H}^{(-)}= & -\tilde{J}^{(-)} \cdot\left(F \frac{\delta}{\delta \tilde{J}^{(-)}}\right) \\
& -\tilde{J}^{(+)} \cdot\left(\frac{\delta}{\delta \tilde{J}^{(-)}} \vee \frac{\delta}{\delta \tilde{J}^{(-)}}\right)-\tilde{J}^{(-)} \cdot\left(\frac{\delta}{\delta \tilde{J}^{(+)}} \vee \frac{\delta}{\delta \tilde{J}^{(-)}}+((+) \leftrightarrow(-))\right) \\
& -\frac{1}{2} g_{\mathrm{St}}^{2} \tilde{J}^{(-)} \cdot\left(\tilde{J}^{(-)} \cdot\left(\wedge \frac{\delta}{\delta \tilde{J}^{(+)}}\right)\right) \\
& -\frac{1}{2} g_{\mathrm{St}}^{2}\left(\tilde{J}^{(+)} \cdot \tilde{J}^{(-)}+((+) \leftrightarrow(-))\right) \cdot\left(\wedge \frac{\delta}{\delta \tilde{J}^{(-)}}\right)
\end{aligned}
$$

where only even number of the (-)-fields appear because of the $\boldsymbol{Z}_{2}$-symmetry of the original Hamiltonian.

The above expression shows that if the coupling is rescaled as $g_{\mathrm{st}}^{2} \rightarrow 2 g_{\mathrm{st}}^{2}$, the form of $\mathcal{H}^{(+)}$becomes completely identical with that of the dual Hamiltonian $\mathcal{H}_{D}$ (4.30) after setting the fields with odd domains to zero. There still remain the portions that do not match between the original and dual theories. They are $\mathcal{H}^{(-)}$in the original theory and the terms containing the fields with odd domains in the dual theory. These contributions give no effects in the tree amplitudes when the initial state does not contain the (-)-fields, in the original theory, because the vacuum expectation value of a single $(-)$-field vanishes due to the $\boldsymbol{Z}_{2}$-symmetry. Similarly, in the dual theory, the string fields with odd domains never appear for the tree amplitudes when the initial state consists only of the fields with even domains. Thus we can conclude that at the level of the tree amplitudes the T-duality

symmetry holds, and that $\tilde{J}_{2 n}^{(+)}$in the original theory are identified with $\tilde{K}_{2 n}$ in the dual theory.

\section{CONCLUDING REMARKS}

We have presented a detailed study of the Schwinger-Dyson equations and the stochastic Hamiltonians for the two-matrix model formulated in both the dual and original variables. We have first performed the duality transformation at the discrete level and then taking the scaling limit separately in both the original and the dual formulations. To deepen our understanding on duality and also to extend our results to more general cases, it is desirable to find a direct transformation of the string fields under the T-dual transformation in the continuum limit.

Our two main results were followings:

1. The theory in the scaling limit is duality symmetric only in the sphere approximation. The absence of the duality symmetry for higher genus originates from the existence 
of nontrivial global $\boldsymbol{Z}_{2}$ vector fields and is explicitly confirmed for the disk amplitude with one handle.

2. We extended the previous discussion on the remarkable commutativity property between the mixing of the string fields in the process of taking the scaling limit and the merging-splitting interactions of the string fields, by proposing a general rule of the string-field mixing.

The first result, which is a consequence of the absence of the momentum-winding symmetry, might be important in thinking about T-duality transformation in string theory for general manifolds without simple target-space isometry. If we want to treat the dual transformed theories in equal footing with the original theories as required once we introduce D-branes, this would suggest that the fundamental variables are worldsheet-vector fields or currents instead of the target space coordinates. Also, if we reformulate the original spin model from the beginning in terms of the link variables instead of the site variables, it may be possible to construct completely duality symmetric models. In fact, it is easy to define such a self-dual lattice model using the same method as a self-dual $X Y$ model as a latticeregularized version of a circle-compactified string theory. This is discussed in Appendix A. A difficulty is that such a model in general requires us to introduce both the site and link variables simultaneously [ in order to include the global winding-like modes before the dual transformation and cannot be described in terms of simply solvable matrix models.

The second result concerning on the universal structure of string field theories might be useful in discussing possible symmetry structure and the background independence of string field theories in general, quite independently of the problem of the operator mixing.

From the viewpoint of our original motivation to the present work, a crucial question is whether this kind of the formulation of string field theory is really possible for the higherdimensional models, especially, including supersymmetry. If we can write down a single Hamiltonian which is supposed to govern all-genus behaviors of the theory at once, it would be a promising starting point for studying the non-perturbative symmetries including S-duality structure.

\section{ACKNOWLEDGMENTS}

The work of T. Y. is partially supported by a US-Japan Collaborative Program from the Japan Society for the Promotion of Science. The work of Y. O. is partially supported by a Predoctoral Research Fellowship from the Japan Society for the Promotion of Science.

\section{APPENDIX A: STRING T-DUALITY AS KRAMERS-WANNIER DUALITY}

In this Appendix, we briefly discuss how the T-duality symmetry of circle-compactified string theories is reformulated as a special case of the Kramers-Wannier duality, namely that

\footnotetext{
${ }^{7}$ For an example of such a model, see [11].
} 
of an XY model with a Villain-type action on a general lattice. For convenience, we will use the familiar notation of square lattice, with $\triangle_{\mu}$ being the directional difference between nearest neighbor sites along the direction $\mu$. Note however that the arguments below are valid for arbitrary lattices with nearest neighbor interactions.

The partition function of the model for a lattice on an arbitrary two-dimensional surface is defined as

$$
Z=\oint[d X]\left(\prod_{s, \mu} \sum_{m_{s, \mu}}\right) \prod_{s} \delta\left(\triangle_{\mu} m_{s, \nu}-\triangle_{\nu} m_{s, \mu}\right) \exp \left[-\frac{R^{2}}{4 \pi} \sum_{s, \mu}\left(\triangle_{\mu} X_{s}-2 \pi m_{s, \mu}\right)^{2}\right]
$$

where the constraint $\triangle_{\mu} m_{s, \nu}-\triangle_{\nu} m_{s, \mu}=0$ is imposed in order to suppress the local vortex excitations which are forbidden in the usual continuum formulation of circle-compactified string theory. The target space coordinate $X$ of the compactification circle is normalized such that the periodicity is $2 \pi$ and the symbol $\oint[d X]$ denotes the path integral with respect to $X$ over a single period. Correspondingly, the variable $m_{s, \mu}$ takes integer values, and the radius parameter $R$ appears in front of the world-sheet action. Thus, the $R^{2}$ just plays the role of the inverse temperature. Note also that, after solving the constraint, the integervalued vector field $m_{s, \mu}$ just represents the winding modes along nontrivial homology cycles for an arbitrary surface of generic genus, since the pure gauge mode of the form $m_{s, \mu}=\triangle_{\mu} m_{s}$ is absorbed into a shift of the target coordinate $X_{s} \rightarrow X_{s}+2 \pi m_{s}$, which in turn ensures the periodicity of the integrand of the partition function after the summation over $m_{s}$.

Now performing the Fourier transformation and introducing auxiliary variable $\tilde{X}_{\tilde{s}}$ to exponentiate the constraint, we have

$$
\begin{gathered}
Z \propto \oint[d X]\left(\prod_{s, \mu} \sum_{m_{s, \mu}}\right) \oint[d \tilde{X}] \int\left(\prod_{\tilde{s}, \mu} d \psi_{\tilde{s}, \mu}\right) \exp \sum_{\tilde{s}}\left[i \tilde{X}_{\tilde{s}} \frac{\epsilon_{\mu \nu}}{2}\left(\triangle_{\mu} m_{s, \nu}-\triangle_{\nu} m_{s, \mu}\right)\right. \\
\left.+i \frac{1}{2 \pi} \epsilon_{\mu \nu} \psi_{\tilde{s}, \mu}\left(\triangle_{\nu} X_{s}-2 \pi m_{s, \nu}\right)-\frac{1}{4 \pi R^{2}} \psi_{\tilde{s}, \mu}^{2}\right]
\end{gathered}
$$

where the integrals for $\tilde{X}_{\tilde{s}}$ is again over a single period $2 \pi$, while the integral for $\psi_{\tilde{s}, \mu}$ is the whole real axis. Note also that all the new variables are introduced on the dual lattice; namely, $\tilde{X}_{\tilde{s}}$ on dual sites, $\psi_{\tilde{s}, \mu}$ on dual links, and the correspondence between the original site $s$ and the dual site $\tilde{s}$ is fixed uniquely in the first and the second terms on the exponential of the integrand. The summation over $m_{s, \mu}$ leads to the constraint

$$
\psi_{\tilde{s}, \mu}=\triangle_{\mu} \tilde{X}_{\tilde{s}}-2 \pi n_{\tilde{s}, \mu}
$$

where $n_{\tilde{s}, \mu}$ defined on each dual link takes arbitrary integer values. After substituting this result and performing the $X$ integral, we obtain the constraint for $n_{\tilde{s}, \mu}$

$$
\triangle_{\mu} n_{\tilde{s}, \nu}-\triangle_{\nu} n_{\tilde{s}, \mu}=0
$$

whose solutions are again the global integer-valued vector fields along nontrivial homology cycles. Thus, the partition function now takes the form

$$
Z \propto \oint[d \tilde{X}]\left(\prod_{\tilde{s}, \mu} \sum_{n_{\tilde{s}, \mu}}\right) \prod_{\tilde{s}} \delta\left(\triangle_{\mu} n_{\tilde{s}, \nu}-\triangle_{\nu} n_{\tilde{s}, \mu}\right) \exp \left[-\frac{1}{4 \pi R^{2}} \sum_{s, \mu}\left(\triangle_{\mu} \tilde{X}_{\tilde{s}}-2 \pi n_{\tilde{s}, \mu}\right)^{2}\right]
$$


which is identical to the original form (A1) with the correspondence $R \leftrightarrow 1 / R$. Note that the integer-valued vector field $n_{\tilde{s}, \mu}$, appearing as the winding mode of the dual coordinate $\tilde{X}_{\tilde{s}}$ can be interpreted as the momentum mode for the original coordinate $X_{s}$. The symmetrical role of the winding $\left(m_{\mu}\right)$ and the momentum modes $\left(n_{\mu}\right)$ for arbitrary genus is precisely the structure of the usual T-duality symmetry.

Obviously, the above model can be easily generalized for $Z(N)$ target space preserving the self-dual structure. However, it is not easy to construct a simply solvable matrix model corresponding to such a lattice model since it requires us to introduce both link and site variables simultaneously.

\section{APPENDIX B: DISK AND CYLINDER AMPLITUDES IN DUAL TWO-MATRIX MODEL}

Here, we obtain various disk and cylinder amplitudes in the dual two-matrix model, by taking the continuum limit of the corresponding Schwinger-Dyson equations. Some of our results have already been derived in Ref. [7]. However, for making this paper self-contained, we will give a brief mention also for those results.

At first, we introduce the following notations for the disk amplitudes:

$$
\begin{aligned}
V_{X^{n} Y^{m}} & =\left\langle\frac{1}{N} \operatorname{tr} X^{n} Y^{m}\right\rangle_{0}, \\
V(\xi) & =\left\langle\frac{1}{N} \operatorname{tr} \frac{1}{\xi-X}\right\rangle_{0}, \\
V_{j}(\xi) & =\left\langle\frac{1}{N} \operatorname{tr} \frac{1}{\xi-X} Y^{j}\right\rangle_{0}, \\
V^{(m)}\left(\xi_{1}, \cdots, \xi_{m}\right) & =\left\langle\frac{1}{N} \operatorname{tr} \frac{1}{\xi_{1}-X} Y \cdots Y \frac{1}{\xi_{m}-X} Y\right\rangle_{0}, \\
V_{j}^{(m)}\left(\xi_{1} ; \xi_{2} \cdots, \xi_{m}\right) & =\left\langle\frac{1}{N} \operatorname{tr} \frac{1}{\xi_{1}-X} Y^{j} \frac{1}{\xi_{2}-X} Y \cdots Y \frac{1}{\xi_{m}-X} Y\right\rangle_{0},
\end{aligned}
$$

and for the cylinder amplitudes:

$$
\begin{aligned}
V_{Y \mid Y}^{\mathrm{cyl}} & =\langle\operatorname{tr} Y \operatorname{tr} Y\rangle_{0}, \\
V_{n \mid m}^{\mathrm{cyl}}(\xi \mid \eta) & =\left\langle\operatorname{tr} \frac{1}{\xi-X} Y^{n} \operatorname{tr} \frac{1}{\eta-X} Y^{m}\right\rangle_{0}, \\
V_{n \mid 1}^{\mathrm{cyl}}\left(\xi_{1} ; \xi_{2}, \cdots, \xi_{m} \mid \eta\right) & =\left\langle\operatorname{tr}\left(\frac{1}{\xi_{1}-X} Y^{n} \frac{1}{\xi_{2}-X} Y \cdots Y \frac{1}{\xi_{m}-X} Y\right) \operatorname{tr} \frac{1}{\eta-X} Y\right\rangle_{0}, \\
V_{n \mid Y^{j}}^{\mathrm{cyl}}\left(\xi_{1} ; \xi_{2}, \cdots, \xi_{m}\right) & =\left\langle\operatorname{tr}\left(\frac{1}{\xi_{1}-X} Y^{n} \frac{1}{\xi_{2}-X} Y \cdots Y \frac{1}{\xi_{m}-X} Y\right) \operatorname{tr} Y^{j}\right\rangle_{0}, \\
V^{\mathrm{cyl}(m \mid 1)}\left(\xi_{1}, \cdots, \xi_{m} \mid \eta_{1}\right) & =\left\langle\operatorname{tr}\left(\frac{1}{\xi_{1}-X} Y \cdots Y \frac{1}{\xi_{m}-X} Y\right) \operatorname{tr} \frac{1}{\eta_{1}-X} Y\right\rangle_{0}, \\
V_{Y^{n}}^{\mathrm{cyl}(m)}\left(\xi_{1}, \cdots, \xi_{m}\right) & =\left\langle\operatorname{tr}\left(\frac{1}{\xi_{1}-X} Y \cdots Y \frac{1}{\xi_{m}-X} Y\right) \operatorname{tr} Y^{n}\right\rangle_{0},
\end{aligned}
$$


where $\langle\cdots\rangle_{0}$ stands for the connected amplitude on the surface with no handle.

\section{B.1. $V(\xi), V_{2}(\xi)$}

As in Ref. [7], the closed equation for $V(\xi)$ is derived by combining the five SchwingerDyson equations obtained from the following identities in the planar limit:

$$
\begin{aligned}
0 & =\int d^{N^{2}} X d^{N^{2}} Y \sum_{\alpha} \frac{\partial}{\partial X_{\alpha}} \operatorname{tr}\left(\frac{1}{\xi-X} t^{\alpha}\right) \mathrm{e}^{-S_{D}}, \\
0 & =\int d^{N^{2}} X d^{N^{2}} Y \sum_{\alpha} \frac{\partial}{\partial Y_{\alpha}} \operatorname{tr}\left(\frac{1}{\xi-X} Y t^{\alpha}\right) \mathrm{e}^{-S_{D}}, \\
0 & =\int d^{N^{2}} X d^{N^{2}} Y \sum_{\alpha} \frac{\partial}{\partial Y_{\alpha}} \operatorname{tr}\left(\frac{1}{\xi-X} Y X t^{\alpha}\right) \mathrm{e}^{-S_{D}}, \\
0 & =\int d^{N^{2}} X d^{N^{2}} Y \sum_{\alpha} \frac{\partial}{\partial X_{\alpha}} \operatorname{tr}\left(\frac{1}{\xi-X} Y t^{\alpha} Y\right) \mathrm{e}^{-S_{D}}, \\
0 & =\int d^{N^{2}} X d^{N^{2}} Y \sum_{\alpha} \frac{\partial}{\partial X_{\alpha}} \operatorname{tr}\left(\frac{1}{\xi-X} Y^{2} t^{\alpha}\right) \mathrm{e}^{-S_{D} .}
\end{aligned}
$$

The result is

$$
\hat{g} V(\xi)^{3}+f_{2} V(\xi)^{2}+f_{1} V(\xi)+f_{0}=0
$$

where

$$
\begin{aligned}
f_{2}= & \hat{g}^{2} \xi^{2}+(5 c-1) \hat{g} \xi-2 c(1+c), \\
f_{1}= & 4 c \hat{g}^{2} \xi^{3}-\left(6 c-2 c^{2}\right) \hat{g} \xi^{2}+2 c\left(1-c^{2}\right) \xi-2 \hat{g}^{2} V_{X}+\hat{g}(1-3 c), \\
f_{0}= & \left(6 c-2 c^{2}-4 c \hat{g} \xi\right) \hat{g} V_{X}-4 c \hat{g}^{2} V_{X^{2}}-4 c \hat{g}^{2} \xi^{2}+\left(6 c-2 c^{2}\right) \hat{g} \xi \\
& \quad-2 c\left(1-c^{2}\right)-\hat{g}^{2} .
\end{aligned}
$$

Since the original and dual theories are connected by the transformation $X \leftrightarrow(A+B) / \sqrt{2}$, $Y \leftrightarrow(A-B) / \sqrt{2}, \hat{g} \leftrightarrow g / \sqrt{2}$, the partition functions of both theories are identical. So the critical points of the couplings $c, g$ are also identical: $c_{*}=\frac{-1+2 \sqrt{7}}{27}, g_{*}=\sqrt{10 c_{*}^{3}}$, and $V_{X}$ and $V_{X^{2}}$ can be written by the amplitudes in the original theory $W_{1}=\left\langle\frac{1}{N} \operatorname{tr} A\right\rangle_{0}$ and $W_{3}=\left\langle\frac{1}{N} \operatorname{tr} A^{3}\right\rangle_{0}$ whose forms in the continuum limit are already known in Ref. [6]:

$$
V_{X}=\sqrt{2} W_{1}, \quad V_{X^{2}}=\frac{1-c^{2}}{c g} W_{1}-\frac{g}{c} W_{3}-\frac{1}{c} .
$$

The critical point $\xi_{*}$ is determined by a similar way as $P_{*}$ in the original theory,

$$
\xi_{*}=\frac{\hat{s}}{\sqrt{5 c_{*}}}, \quad \hat{s}=1+\sqrt{7}
$$

Introducing the lattice spacing $a$ and the variables in the continuum theory as

$$
\hat{g}=\hat{g}_{*}\left(1-a^{2} \frac{\hat{s}^{2}}{10} \hat{T}\right), \quad \xi=\xi_{*}(1+a y),
$$


the continuum limit of the solution of (B2) is given by

$$
\begin{aligned}
V(\xi) & =V^{\text {non }}(\xi)+\hat{V}(\xi), \\
V^{\text {non }}(\xi) & =-\frac{f_{2}}{3 \hat{g}} \\
\hat{V}(\xi) & =a^{4 / 3} \frac{c^{1 / 2} \hat{s}^{4 / 3}}{2^{2 / 3} \sqrt{5}}\left[\left(y+\sqrt{y^{2}-\hat{T}}\right)^{4 / 3}+\left(y-\sqrt{y^{2}-\hat{T}}\right)^{4 / 3}\right]+O\left(a^{5 / 3}\right) \\
& \equiv a^{4 / 3} \frac{c^{1 / 2} \hat{s}^{4 / 3}}{2^{2 / 3} \sqrt{5}} v(y)+O\left(a^{5 / 3}\right),
\end{aligned}
$$

where $V^{\text {non }}$ and $\hat{V}$ denote the non-universal and universal parts, respectively. Here and below, $c$ appearing in the formulas in the continuum limit is understood to be fixed at the critical point $c_{*}$.

Also, $V_{2}(\xi)$ can be derived in the same way as in the original theory:

$$
\begin{aligned}
V_{2}(\xi)= & V_{2}^{\mathrm{non}}(\xi)+\hat{V}_{2}(\xi), \\
V_{2}^{\mathrm{non}}(\xi)= & \frac{1}{\hat{g}}\left[\left((1-c-\hat{g} \xi) \xi-V^{\mathrm{non}}(\xi)\right) V^{\mathrm{non}}(\xi)+\hat{g} V_{X}^{\mathrm{non}}-1+c+\hat{g} \xi\right. \\
& \left.+\left((1-c-\hat{g} \xi) \xi-2 V^{\mathrm{non}}(\xi)\right) \hat{V}(\xi)\right], \\
\hat{V}_{2}(\xi)= & -\frac{1}{\hat{g}} \hat{V}(\xi)^{2}+\hat{V}_{X} \equiv a^{8 / 3} \frac{\hat{s}^{8 / 3}}{2^{4 / 3} \cdot 5 c^{1 / 2}} v_{2}(y)+O\left(a^{3}\right), \\
v_{2}(y)= & -v(y)^{2}+\frac{3}{2} \hat{T}^{4 / 3},
\end{aligned}
$$

where the non-universal and universal parts of $V_{X}: V_{X}^{\text {non }}$ and $\hat{V}_{X}$ are known from those of $W_{1}$ via $(\mathrm{B} 3)$. The $y$-independent constant in $\hat{V}_{2}(\xi)$ is different from that in the corresponding amplitude $\left(\hat{W}_{1}(\zeta)\right)$ of the original theory. However, in the rule for identifying the nonuniversal and universal parts, the term $\hat{V}_{X}$, which can be seen as an amplitude with a simpler spin configuration than $\hat{V}_{2}(\xi)$, is allowed to be absorbed in the non-universal part. After adopting such a convention for both original and dual theories, there are no real differences. In fact, since the operator corresponding to this amplitude is always accompanied by $\xi$ derivative in the leading contribution of the Hamiltonian, this change does not affect the continuum Hamiltonian.

$$
\text { B.2. } V^{(2)}\left(\xi_{1}, \xi_{2}\right), V^{(4)}\left(\xi_{1}, \xi_{2}, \xi_{3}, \xi_{4}\right)
$$

For the higher amplitude $V^{(2 k)}$, we can derive the dual version of Staudacher's recursion relation (Eq. (20) in Ref. [12]). Here, we consider the continuum limit for the cases $k=1$ and 2. The corresponding Schwinger-Dyson equations are

$$
\begin{aligned}
V^{(2)}\left(\xi_{1}, \xi_{2}\right) & =\frac{V\left(\xi_{1}\right) V\left(\xi_{2}\right)-\hat{g}\left(V_{2}\left(\xi_{1}\right)+V_{2}\left(\xi_{2}\right)\right)}{1+c-\hat{g}\left(\xi_{1}+\xi_{2}\right)}, \\
V^{(4)}\left(\xi_{1}, \xi_{2}, \xi_{3}, \xi_{4}\right)= & \frac{1}{1+c-\hat{g}\left(\xi_{1}+\xi_{4}\right)}
\end{aligned}
$$




$$
\begin{aligned}
\times & {\left[\left(\left(1-c-\hat{g} \xi_{1}\right) \xi_{1}-V\left(\xi_{1}\right)-V\left(\xi_{3}\right)-V\left(\xi_{4}\right)\right) D_{z}\left(\xi_{1}, \xi_{3}\right) V^{(2)}\left(z, \xi_{2}\right)\right.} \\
& +\left(\left(1-c-\hat{g} \xi_{2}\right) \xi_{2}-V\left(\xi_{1}\right)-V\left(\xi_{2}\right)-V\left(\xi_{4}\right)\right) D_{z}\left(\xi_{2}, \xi_{4}\right) V^{(2)}\left(z, \xi_{3}\right) \\
& +\left(1-c-\hat{g}\left(\xi_{1}+\xi_{3}\right)\right) V^{(2)}\left(\xi_{2}, \xi_{3}\right)+\left(1-c-\hat{g}\left(\xi_{2}+\xi_{4}\right)\right) V^{(2)}\left(\xi_{3}, \xi_{4}\right) \\
& \left.+\hat{g} V_{2}\left(\xi_{2}\right)+\hat{g} V_{2}\left(\xi_{3}\right)\right] .
\end{aligned}
$$

Putting $\xi_{i}=\xi_{*}\left(1+a y_{i}\right)$ and expanding with respect to $a$, the result of $V^{(2)}$ becomes

$$
\begin{aligned}
V^{(2)}\left(\xi_{1}, \xi_{2}\right)= & V^{(2) \text { non }}\left(\xi_{1}, \xi_{2}\right)+\hat{V}^{(2)}\left(\xi_{1}, \xi_{2}\right) \\
V^{(2) \text { non }}\left(\xi_{1}, \xi_{2}\right)= & \frac{1}{5}(1+4 \hat{s})-2 \sqrt{\frac{c}{5}}\left(\xi_{1}+\xi_{2}\right)+\frac{2}{\sqrt{5 c}}\left(\hat{V}\left(\xi_{1}\right)+\hat{V}\left(\xi_{2}\right)\right), \\
\hat{V}^{(2)}\left(\xi_{1}, \xi_{2}\right)= & a^{5 / 3} \frac{\hat{s}^{5 / 3}}{10 \cdot 2^{1 / 3}} \frac{-\hat{v}\left(y_{1}\right)^{2}-\hat{v}\left(y_{2}\right)^{2}-\hat{v}\left(y_{1}\right) \hat{v}\left(y_{2}\right)+3 \hat{T}^{4 / 3}}{y_{1}+y_{2}} \\
& +a^{2} \frac{2 \hat{s}}{75}\left(18 \hat{T}+10 \hat{s}\left(y_{1}^{2}+y_{2}^{2}\right)-15 \hat{s} y_{1} y_{2}\right)+O\left(a^{7 / 3}\right) \\
\equiv & a^{5 / 3} \frac{\hat{s}^{5 / 3}}{10 \cdot 2^{1 / 3}} v^{(2)}\left(y_{1}, y_{2}\right)+O\left(a^{2}\right)
\end{aligned}
$$

where $\hat{v}(y)$ is defined by the rescaling of $\hat{V}(\xi)$ :

$$
\hat{V}(\xi)=a^{4 / 3} \frac{c^{1 / 2} \hat{s}^{4 / 3}}{2^{2 / 3} \sqrt{5}} \hat{v}(y),
$$

and

$$
v^{(2)}\left(y_{1}, y_{2}\right)=\frac{-v\left(y_{1}\right)^{2}-v\left(y_{2}\right)^{2}-v\left(y_{1}\right) v\left(y_{2}\right)+3 \hat{T}^{4 / 3}}{y_{1}+y_{2}} .
$$

Using this and repeating the same procedure for $k=2$, we have

$$
\begin{aligned}
& V^{(4)}\left(\xi_{1}, \xi_{2}, \xi_{3}, \xi_{4}\right)=V^{(4) \operatorname{non}}\left(\xi_{1}, \xi_{2}, \xi_{3}, \xi_{4}\right)+\hat{V}^{(4)}\left(\xi_{1}, \xi_{2}, \xi_{3}, \xi_{4}\right), \\
& V^{(4) \operatorname{non}}\left(\xi_{1}, \xi_{2}, \xi_{3}, \xi_{4}\right)=1-\frac{4}{5 c}\left[D_{z}\left(\xi_{1}, \xi_{3}\right) \hat{V}(z)+D_{z}\left(\xi_{2}, \xi_{4}\right) \hat{V}(z)\right] \\
& -\frac{2}{\sqrt{5 c}}\left[D_{z}\left(\xi_{1}, \xi_{3}\right)\left(\hat{V}^{(2)}\left(z, \xi_{2}\right)+\hat{V}^{(2)}\left(z, \xi_{4}\right)\right)\right. \\
& \left.+D_{z}\left(\xi_{2}, \xi_{4}\right)\left(\hat{V}^{(2)}\left(\xi_{1}, z\right)+\hat{V}^{(2)}\left(\xi_{3}, z\right)\right)\right] \\
& \hat{V}^{(4)}\left(\xi_{1}, \xi_{2}, \xi_{3}, \xi_{4}\right)=a \frac{\hat{s}}{20} v^{(4)}\left(y_{1}, y_{2}, y_{3}, y_{4}\right)+O\left(a^{4 / 3}\right) \\
& =a \frac{\hat{s}}{20}\left\{-\frac{16}{3}\left(y_{1}+y_{2}+y_{3}+y_{4}\right)+\frac{1}{2} \frac{1}{y_{1}-y_{3}} \frac{1}{y_{2}-y_{4}}\right. \\
& \times\left[-v^{(2)}\left(y_{1}, y_{2}\right)\left(v\left(y_{1}\right)+v\left(y_{2}\right)+2 v\left(y_{3}\right)+2 v\left(y_{4}\right)\right)\right. \\
& +v^{(2)}\left(y_{1}, y_{4}\right)\left(v\left(y_{1}\right)+2 v\left(y_{2}\right)+2 v\left(y_{3}\right)+v\left(y_{4}\right)\right) \\
& +v^{(2)}\left(y_{2}, y_{3}\right)\left(2 v\left(y_{1}\right)+v\left(y_{2}\right)+v\left(y_{3}\right)+2 v\left(y_{4}\right)\right) \\
& \left.\left.-v^{(2)}\left(y_{3}, y_{4}\right)\left(2 v\left(y_{1}\right)+2 v\left(y_{2}\right)+v\left(y_{3}\right)+v\left(y_{4}\right)\right)\right]\right\} \\
& +O\left(a^{4 / 3}\right) \text {. }
\end{aligned}
$$

$\hat{V}^{(2)}\left(\xi_{1}, \xi_{2}\right)$ has the same form as the corresponding; amplitude $\hat{W}^{(2)}(\zeta, \sigma)$ in the original theory, which has been pointed out in Ref. [7]. Also, $\hat{V}^{(4)}\left(\xi_{1}, \xi_{2}, \xi_{3}, \xi_{4}\right)$ is the same as 
$\hat{W}^{(4)}\left(\zeta_{1}, \sigma_{1}, \zeta_{2}, \sigma_{2}\right)$, except the linear term of $y_{i}$. Since this term is analytic with respect to $y_{i}$ 's, it is allowed to be absorbed to the non-universal part. So, the difference can be regarded as non-universal.

\section{B.3. $V_{2}^{(3)}\left(\xi_{1}, \xi_{2}, \xi_{3} ;\right)$}

This amplitude can be obtained from the Schwinger-Dyson equation:

$$
0=\int d^{N^{2}} X d^{N^{2}} Y \sum_{\alpha} \frac{\partial}{\partial X_{\alpha}} \operatorname{tr}\left(\frac{1}{\xi_{1}-X} Y \frac{1}{\xi_{2}-X} Y \frac{1}{\xi_{3}-X} t^{\alpha}\right) \mathrm{e}^{-S_{D}} .
$$

A similar calculation as before leads to the result

$$
\begin{aligned}
V_{2}^{(3)}\left(\xi_{1}, \xi_{2}, \xi_{3} ;\right)= & V_{2}^{(3) \text { non }}\left(\xi_{1}, \xi_{2}, \xi_{3} ;\right)+\hat{V}_{2}^{(3)}\left(\xi_{1}, \xi_{2}, \xi_{3} ;\right), \\
V_{2}^{(3) \text { non }}\left(\xi_{1}, \xi_{2}, \xi_{3} ;\right)= & \frac{1}{\hat{g}}\left(V^{\text {non }}\left(\xi_{1}\right)+V^{\text {non }}\left(\xi_{3}\right)-\left(1-c-\hat{g} \xi_{3}\right) \xi_{3}\right) D_{z}\left(\xi_{1}, \xi_{3}\right) V^{(2)}\left(z, \xi_{2}\right) \\
& -2 \sqrt{\frac{c}{5} \frac{1}{\hat{g}}}\left(\hat{V}\left(\xi_{1}\right)+\hat{V}\left(\xi_{3}\right)\right)-\frac{2}{\sqrt{5 c}} D_{z}\left(\xi_{1}, \xi_{3}\right) \hat{V}_{2}(z) \\
& -V_{2}\left(\xi_{2}\right)-\frac{1}{\hat{g}}\left(1-c-\hat{g}\left(\xi_{1}+\xi_{3}\right)\right) V^{(2)}\left(\xi_{1}, \xi_{2}\right), \\
\hat{V}_{2}^{(3)}\left(\xi_{1}, \xi_{2}, \xi_{3} ;\right)= & a^{2} \frac{\hat{s}^{2}}{20 \sqrt{5 c}}\left(v\left(y_{1}\right)+v\left(y_{3}\right)\right) D_{z}\left(y_{1}, y_{3}\right) v^{(2)}\left(z, y_{2}\right)+O\left(a^{7 / 3}\right) \\
\equiv & a^{2} \frac{\hat{s}^{2}}{20 \sqrt{5 c}} v_{2}^{(3)}\left(y_{1}, y_{2}, y_{3} ;\right)+O\left(a^{7 / 3}\right) .
\end{aligned}
$$

\section{B.4. $V_{Y \mid Y}^{\mathrm{cyl}}, V_{Y}^{\mathrm{cyl}(1)}(\xi), V_{1 \mid 1}^{\mathrm{cyl}}\left(\xi \mid \xi^{\prime}\right)$}

Let us next consider the cylinder amplitudes. As presented in $[7], V_{Y \mid Y}^{\text {cyl }}$ and $V_{Y}^{\text {cyl }}{ }^{(1)}(\xi)$ can be obtained from the single Schwinger-Dyson equation

$$
V(\xi)-(1+c-2 \hat{g} \xi) V_{Y}^{\mathrm{cyl}(1)}(\xi)-2 \hat{g} V_{Y \mid Y}^{\mathrm{cyl}}=0
$$

by using the fact that the coefficient of $V_{Y}^{\text {cyl }}{ }^{(1)}(\xi)$ vanishes at $\xi=\frac{1+c}{2 \hat{g}}=\xi_{*}+O\left(a^{2}\right)$. The results are

$$
\begin{aligned}
V_{Y \mid Y}^{\mathrm{cyl}} & =\frac{1}{2 \hat{g}} V\left(\frac{1+c}{2 \hat{g}}\right) \\
& =\frac{1}{5 c}-a^{4 / 3} \frac{\hat{s}^{4 / 3}}{5 \cdot 2^{5 / 3} c} \hat{T}^{2 / 3}+O\left(a^{2}\right), \\
V_{Y}^{\mathrm{cyl}(1)}(\xi) & =\frac{1}{\sqrt{5 c}}-a^{1 / 3} \frac{\hat{s}^{1 / 3}}{2^{5 / 3} \sqrt{5 c}} \frac{\hat{v}(y)+\hat{T}^{2 / 3}}{y}+O(a) \\
& \equiv \frac{1}{\sqrt{5 c}}+\hat{V}_{Y}^{\mathrm{cyl}(1)}(\xi) .
\end{aligned}
$$


For $V_{1 \mid 1}^{\text {cyl }}\left(\xi \mid \xi^{\prime}\right)$, considering the Schwinger-Dyson equation obtained from the identity

$$
0=\int d^{N^{2}} X d^{N^{2}} Y \sum_{\alpha} \frac{\partial}{\partial Y_{\alpha}}\left(\operatorname{tr}\left(\frac{1}{\xi-X} t^{\alpha}\right) \operatorname{tr}\left(\frac{1}{\xi^{\prime}-X} Y\right) \mathrm{e}^{-S_{D}}\right)
$$

we have

$$
\begin{aligned}
V_{1 \mid 1}^{\mathrm{cyl}}\left(\xi \mid \xi^{\prime}\right) & =\frac{-D_{z}\left(\xi, \xi^{\prime}\right) V(z)-2 \hat{g} V_{Y}^{\mathrm{cyl}(1)}\left(\xi^{\prime}\right)}{1+c-2 \hat{g} \xi} \\
& =a^{-2 / 3} \frac{1}{2^{5 / 3} \hat{s}^{2 / 3}} \frac{1}{y y^{\prime}}\left(\frac{y^{\prime} v(y)-y v\left(y^{\prime}\right)}{y-y^{\prime}}-\hat{T}^{2 / 3}\right)+O\left(a^{0}\right) .
\end{aligned}
$$

B.5. $V_{2 \mid Y}^{\text {cyl }}\left(\xi_{1} ; \xi_{2}\right), V_{Y}^{\text {cyl }{ }^{(3)}}\left(\xi_{1}, \xi_{2}, \xi_{3}\right)$

We start with the following two identities:

$$
\begin{aligned}
& 0=\int d^{N^{2}} X d^{N^{2}} Y \sum_{\alpha} \frac{\partial}{\partial X_{\alpha}} \operatorname{tr}\left(\frac{1}{\xi_{1}-X} t^{\alpha} \frac{1}{\xi_{2}-X} Y\right) \operatorname{tr} Y \mathrm{e}^{-S_{D}}, \\
& 0=\int d^{N^{2}} X d^{N^{2}} Y \sum_{\alpha} \frac{\partial}{\partial Y_{\alpha}} \operatorname{tr}\left(\frac{1}{\xi_{1}-X} Y \frac{1}{\xi_{2}-X} Y \frac{1}{\xi_{3}-X} t^{\alpha}\right) \operatorname{tr} Y \mathrm{e}^{-S_{D}} .
\end{aligned}
$$

The amplitudes $V_{2 \mid Y}^{\text {cyl }}\left(\xi_{1} ; \xi_{2}\right)$ and $V_{Y}^{\text {cyl }}{ }^{(3)}\left(\xi_{1}, \xi_{2}, \xi_{3}\right)$ can be obtained by using the SchwingerDyson equations derived from the above identities.

The results are as follows:

$$
\begin{aligned}
& V_{2 \mid Y}^{\mathrm{cyl}}\left(\xi_{1} ; \xi_{2}\right)=V_{2 \mid Y}^{\mathrm{cyl} \text { non }}\left(\xi_{1} ; \xi_{2}\right)+\hat{V}_{2 \mid Y}^{\mathrm{cyl}}\left(\xi_{1} ; \xi_{2}\right), \\
& V_{2 \mid Y}^{\text {cyl non }}\left(\xi_{1} ; \xi_{2}\right)=\frac{1}{\hat{g}}\left(V^{\text {non }}\left(\xi_{1}\right)+V^{\text {non }}\left(\xi_{2}\right)-\left(1-c-\hat{g} \xi_{1}\right) \xi_{1}\right) D_{z}\left(\xi_{1}, \xi_{2}\right) V_{Y}^{\text {cyl }{ }^{(1)}}(z) \\
& -V_{Y \mid Y}^{\mathrm{cyl}}-\frac{1}{\hat{g}}\left(1-c-\hat{g}\left(\xi_{1}+\xi_{2}\right)\right) V_{Y}^{\mathrm{cyl}(1)}\left(\xi_{2}\right), \\
& \hat{V}_{2 \mid Y}^{\mathrm{cyl}}\left(\xi_{1} ; \xi_{2}\right)=-a^{2 / 3} \frac{\hat{s}^{2 / 3}}{2^{7 / 3} \cdot 5 c}\left(v\left(y_{1}\right)+v\left(y_{2}\right)\right) D_{z}\left(y_{1}, y_{2}\right) \frac{v(z)+\hat{T}^{2 / 3}}{z} \\
& +O(a)
\end{aligned}
$$

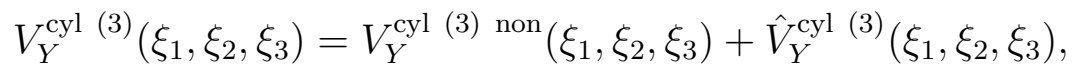

$$
\begin{aligned}
& V_{Y}^{\text {cyl (3) non }}\left(\xi_{1}, \xi_{2}, \xi_{3}\right)=\frac{2}{\sqrt{5 c}}\left[-D_{z}\left(\xi_{1}, \xi_{2}\right)-D_{z}\left(\xi_{2}, \xi_{3}\right)-D_{z}\left(\xi_{3}, \xi_{1}\right)\right] \hat{V}_{Y}^{\text {cyl }}{ }^{(1)}(z) \text {, } \\
& \hat{V}_{Y}^{\text {cyl }{ }^{(3)}}\left(\xi_{1}, \xi_{2}, \xi_{3}\right)=a^{-1 / 3} \frac{\hat{s}^{-1 / 3}}{4 \cdot 2^{1 / 3} \sqrt{5 c}}\left[\frac{\hat{T}^{2 / 3}}{y_{1} y_{2} y_{3}} v\left(y_{1}\right)-\frac{2 \hat{T}^{4 / 3}}{\left(y_{1}+y_{2}\right)\left(y_{2}+y_{3}\right)\left(y_{3}+y_{1}\right)}\right. \\
& -v\left(y_{1}\right) v\left(y_{2}\right)\left(\frac{1}{y_{1}\left(y_{1}-y_{3}\right)}+\frac{1}{y_{2}\left(y_{2}-y_{3}\right)}\right) \frac{1}{y_{1}+y_{2}} \\
& -v\left(y_{1}\right)^{2} \frac{3 y_{1}^{2}-y_{1} y_{2}-y_{2} y_{3}-y_{3} y_{1}}{y_{1}\left(y_{1}^{2}-y_{2}^{2}\right)\left(y_{1}^{2}-y_{3}^{2}\right)} \\
& \left.+ \text { cyclic permutations of } y_{1}, y_{2}, y_{3}\right]+O\left(a^{0}\right) \text {. }
\end{aligned}
$$




\section{B.6. $V_{Y}^{\text {cyl }{ }^{(5)}}\left(\xi_{1}, \xi_{2}, \xi_{3}, \xi_{4}, \xi_{5}\right)$}

Here, we show only the result of the non-universal part of this amplitude in order to examine the mixing among the operators with odd domains. It is necessary to consider the two Schwinger-Dyson equations originating from

$$
\begin{aligned}
& 0=\int d^{N^{2}} X d^{N^{2}} Y \sum_{\alpha} \frac{\partial}{\partial Y_{\alpha}}\left[\operatorname{tr}\left(\frac{1}{\xi_{1}-X} Y \frac{1}{\xi_{2}-X} Y \frac{1}{\xi_{3}-X} Y \frac{1}{\xi_{4}-X} Y \frac{1}{\xi_{5}-X} t^{\alpha}\right) \operatorname{tr} Y \mathrm{e}^{-S_{D}}\right], \\
& 0=\int d^{N^{2}} X d^{N^{2}} Y \sum_{\alpha} \frac{\partial}{\partial X_{\alpha}}\left[\operatorname{tr}\left(\frac{1}{\xi_{1}-X} Y \frac{1}{\xi_{2}-X} Y \frac{1}{\xi_{3}-X} Y \frac{1}{\xi_{4}-X} t^{\alpha}\right) \operatorname{tr} Y \mathrm{e}^{-S_{D}}\right] .
\end{aligned}
$$

Combining these, we obtain

$$
\begin{aligned}
& V_{Y}^{\text {cyl }}{ }^{(5)}\left(\xi_{1}, \cdots, \xi_{5}\right)=\frac{1}{1+c-\hat{g}\left(\xi_{1}+\xi_{5}\right)} \\
& \times\left[\left(\left(1-c-\hat{g} \xi_{5}\right) \xi_{5}-V\left(\xi_{1}\right)-V\left(\xi_{2}\right)-V\left(\xi_{5}\right)\right) D_{z}\left(\xi_{2}, \xi_{5}\right) V_{Y}^{\mathrm{cyl}(3)}\left(z, \xi_{3}, \xi_{4}\right)\right. \\
& \quad+\left(\left(1-c-\hat{g} \xi_{4}\right) \xi_{4}-V\left(\xi_{1}\right)-V\left(\xi_{4}\right)-V\left(\xi_{5}\right)\right) D_{z}\left(\xi_{1}, \xi_{4}\right) V_{Y}^{\mathrm{cyl}(3)}\left(z, \xi_{2}, \xi_{3}\right) \\
& \quad+\left(D_{z}\left(\xi_{3}, \xi_{5}\right) V^{(2)}\left(z, \xi_{4}\right)+D_{z}\left(\xi_{2}, \xi_{4}\right) V^{(2)}\left(z, \xi_{3}\right)\right) D_{w}\left(\xi_{1}, \xi_{2}\right) V_{Y}^{\mathrm{cyl}(1)}(w) \\
& \quad+\left(D_{z}\left(\xi_{1}, \xi_{3}\right) V^{(2)}\left(z, \xi_{2}\right)+D_{z}\left(\xi_{2}, \xi_{4}\right) V^{(2)}\left(z, \xi_{3}\right)\right) D_{w}\left(\xi_{4}, \xi_{5}\right) V_{Y}^{\mathrm{cyl}(1)}(w) \\
& \quad+\left(D_{z}\left(\xi_{1}, \xi_{3}\right) V^{(2)}\left(z, \xi_{2}\right)+V\left(\xi_{3}\right)+V\left(\xi_{4}\right)-\left(1-c-\hat{g} \xi_{4}\right) \xi_{4}\right) D_{w}\left(\xi_{3}, \xi_{4}\right) V_{Y}^{\mathrm{cyl}}(1)(w) \\
& \quad+\left(D_{z}\left(\xi_{3}, \xi_{5}\right) V^{(2)}\left(z, \xi_{4}\right)+V\left(\xi_{2}\right)+V\left(\xi_{3}\right)-\left(1-c-\hat{g} \xi_{3}\right) \xi_{3}\right) D_{w}\left(\xi_{2}, \xi_{3}\right) V_{Y}^{\mathrm{cyl}(1)}(w) \\
& \quad+\left(1-c-\hat{g}\left(\xi_{1}+\xi_{4}\right)\right) V_{Y}^{\mathrm{cyl}(3)}\left(\xi_{1}, \xi_{2}, \xi_{3}\right)+\left(1-c-\hat{g}\left(\xi_{2}+\xi_{5}\right)\right) V_{Y}^{\mathrm{cyl}(3)}\left(\xi_{2}, \xi_{3}, \xi_{4}\right) \\
& \quad-\left(1-c-\hat{g}\left(\xi_{2}+\xi_{3}\right)\right) V_{Y}^{\mathrm{cyl}(1)}\left(\xi_{2}\right)-\left(1-c-\hat{g}\left(\xi_{3}+\xi_{4}\right)\right) V_{Y}^{\mathrm{cyl}(1)}\left(\xi_{3}\right) \\
& \left.\quad-D_{z}\left(\xi_{1}, \xi_{5}\right) V^{(4)}\left(z, \xi_{2}, \xi_{3}, \xi_{4}\right)-2 \hat{g} V_{Y \mid Y}^{\mathrm{cyl}}\right]
\end{aligned}
$$

In the continuum limit, the non-universal part $V_{Y}^{\text {cyl (5) non }}$ becomes

$$
\begin{aligned}
& V_{Y}^{\text {cyl }{ }^{(5)}}\left(\xi_{1}, \cdots, \xi_{5}\right)=V_{Y}^{\text {cyl }}{ }^{(5)} \text { non }\left(\xi_{1}, \cdots, \xi_{5}\right)+\hat{V}_{Y}^{\text {cyl }}{ }^{(5)}\left(\xi_{1}, \cdots, \xi_{5}\right) \\
& V_{Y}^{\text {cyl }(5) \text { non }}\left(\xi_{1}, \cdots, \xi_{5}\right)=\frac{4}{5 c}\left[D_{z}\left(\xi_{1}, \xi_{3}\right) D_{w}\left(z, \xi_{5}\right)+D_{z}\left(\xi_{1}, \xi_{3}\right) D_{w}\left(z, \xi_{4}\right)\right. \\
& +D_{z}\left(\xi_{2}, \xi_{4}\right) D_{w}\left(z, \xi_{5}\right)+D_{z}\left(\xi_{1}, \xi_{2}\right) D_{w}\left(z, \xi_{4}\right) \\
& \left.+D_{z}\left(\xi_{2}, \xi_{3}\right) D_{w}\left(z, \xi_{5}\right)\right] \hat{V}_{Y}^{\mathrm{cyl}(1)}(w) \\
& -\frac{2}{\sqrt{5 c}}\left[D_{z}\left(\xi_{1}, \xi_{3}\right) \hat{V}_{Y}^{\mathrm{cyl}(3)}\left(z, \xi_{4}, \xi_{5}\right)+D_{z}\left(\xi_{2}, \xi_{4}\right) \hat{V}_{Y}^{\mathrm{cyl}(3)}\left(\xi_{1}, z, \xi_{5}\right)\right.
\end{aligned}
$$

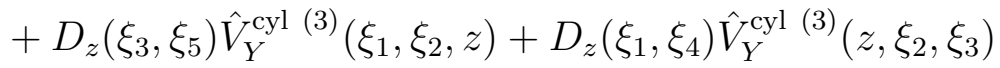

$$
\begin{aligned}
& \left.+D_{z}\left(\xi_{2}, \xi_{5}\right) \hat{V}_{Y}^{\text {cyl }}{ }^{(3)}\left(z, \xi_{3}, \xi_{4}\right)\right] \text {, } \\
& \hat{V}_{Y}^{\text {cyl }}{ }^{(5)}\left(\xi_{1}, \cdots, \xi_{5}\right)=O\left(a^{-1}\right) \text {. }
\end{aligned}
$$

From the results until now, we can read off the scaling property of the universal parts of the string fields as follows. First, the universal part of $\operatorname{tr} Y$ scales as $O\left(a^{2 / 3}\right)$. Since the partition functions both in the original and dual theories are identical, the double scaling limits are also $\left(\frac{1}{N}=O\left(a^{7 / 3}\right)\right.$ in both theories). Using this, it can be seen that the string 
fields scale as $\hat{\Psi}_{X}=O\left(a^{4 / 3}\right), \hat{\Psi}_{n}=O\left(a^{7 / 3-n / 3}\right)$, where $n=1,2,3, \cdots$. This coincides with the interpretation of the boundary conformal field theory [13] as in the original theory.

\section{APPENDIX C: CONTINUUM SPIN-FLIP OPERATOR IN THE DUAL FORMALISM}

Similarly as in the original theory, a microscopic domain consisting only of a single flipped spin can be obtained by an integration $\int_{C} \frac{d y}{2 \pi i}$ with respect to $y$ of the macroscopic domain. We will check this in the case of the disk and cylinder amplitudes.

First, for the disk amplitudes the following relations can be verified by direct calculations using the results in Appendix B:

$$
\begin{aligned}
\partial_{y_{2}} \int_{C} \frac{d y_{1}}{2 \pi i} v^{(2)}\left(y_{1}, y_{2}\right) & =\partial_{y_{2}} v_{2}\left(y_{2}\right), \\
\int_{C} \frac{d y_{1}}{2 \pi i} v^{(4)}\left(y_{1}, y_{2}, y_{3}, y_{4}\right) & =v_{2}^{(3)}\left(y_{2}, y_{3}, y_{4} ;\right),
\end{aligned}
$$

where the rule of the integral is identical with that in the original theory. The contour $C$ wraps around the negative real axis and the singularities in the left half plane, and the unintegrated variables, for example $y_{2}, y_{3}, y_{4}$ in the above, are understood to be outside the contour, while $-y_{2},-y_{3},-y_{4}$ to be inside the contour.

By including the overall normalizations, (C1) can be written as

$$
\begin{aligned}
\partial_{\xi_{2}} \oint \frac{d \xi_{1}}{2 \pi i} \hat{V}^{(2)}\left(\xi_{1}, \xi_{2}\right) & =\partial_{\xi_{2}} \hat{V}_{2}\left(\xi_{2}\right)+O\left(a^{2}\right), \\
\hat{\oint} \frac{d \xi_{1}}{2 \pi i} \hat{V}^{(4)}\left(\xi_{1}, \xi_{2}, \xi_{3}, \xi_{4}\right) & =\hat{V}_{2}^{(3)}\left(\xi_{2}, \xi_{3}, \xi_{4} ;\right)+O\left(a^{7 / 3}\right),
\end{aligned}
$$

where the integral symbol $\hat{\oint} \frac{d \xi}{2 \pi i}$ is used in the sense of

$$
\hat{\oint} \frac{d \xi}{2 \pi i}=a \xi_{*} \int_{C} \frac{d y}{2 \pi i}
$$

In contrast to the original theory, no additional factor of a finite renormalization appears here.

Similarly, for the cylinder amplitudes, we can show that the following formulas hold:

$$
\begin{aligned}
\hat{\oint} \frac{d \xi}{2 \pi i} V_{1 \mid 1}^{\mathrm{cyl}}\left(\xi \mid \xi^{\prime}\right) & =\hat{V}_{Y}^{\mathrm{cyl}(1)}\left(\xi^{\prime}\right)+O\left(a^{2 / 3}\right), \\
\hat{\oint} \frac{d \xi}{2 \pi i} \hat{V}_{Y}^{\mathrm{cyl}(3)}\left(\xi_{1}, \xi, \xi_{2}\right) & =\hat{V}_{2 \mid Y}^{\mathrm{cyl}}\left(\xi_{1} ; \xi_{2}\right)+O(a) .
\end{aligned}
$$




\section{REFERENCES}

[1] H. A. Kramers and G. H. Wannier, Phys. Rev. 60, 252 (1941).

[2] A. Shapere and F. Wilczek, Nucl. Phys. B320, 669 (1989) and references therein.

[3] K. Kikkawa and M. Yamasaki, Phys. Lett. 149B, 357 (1984).

[4] For a review, see J. Polchinski, S. Chaudhuri and C. V. Johnson, preprint hepth/9602052.

[5] N. Ishibashi and H. Kawai, Phys. Lett. 314B, 190 (1993).

[6] F. Sugino and T. Yoneya, Phys. Rev. D53, 4448 (1996).

[7] S. M. Carroll, M. E. Ortiz and W. Taylor IV, Nucl. Phys. B468 [FS], 420 and 383 (1996).

[8] A. Jevicki and J. Rodrigues, Nucl. Phys. B421, 278 (1994).

[9] I. Kostov, Mod. Phys. Lett. A4, 217 (1989).

B. Duplantier and I. Kostov, Nucl. Phys. B340, 491 (1990).

[10] T. Kugo and B. Zwiebach, Prog. Theor. Phys. 87, 801 (1992).

[11] V. A. Kazakov, M. Staudacher and T. Wynter, Comm. Math. Phys. 177, 451 (1996).

[12] M. Staudacher, Phys. Lett. 305B, 332 (1993).

[13] J. L. Cardy, Nucl. Phys. B275 [FS17], 200 (1986). 